San Jose State University

SJSU ScholarWorks

Master's Theses

Master's Theses and Graduate Research

1996

\title{
Effects of housing density on mouse physiology and behavior in NASA animal enclosure module simulators
}

Sujata Naidu

San Jose State University

Follow this and additional works at: https://scholarworks.sjsu.edu/etd_theses

\section{Recommended Citation}

Naidu, Sujata, "Effects of housing density on mouse physiology and behavior in NASA animal enclosure module simulators" (1996). Master's Theses. 1395.

DOI: https://doi.org/10.31979/etd.ac7z-s4en

https://scholarworks.sjsu.edu/etd_theses/1395

This Thesis is brought to you for free and open access by the Master's Theses and Graduate Research at SJSU ScholarWorks. It has been accepted for inclusion in Master's Theses by an authorized administrator of SJSU ScholarWorks. For more information, please contact scholarworks@sjsu.edu. 


\section{INFORMATION TO USERS}

This manuscript has been reproduced from the microfilm master. UMI films the text directly from the original or copy submitted. Thus, some thesis and dissertation copies are in typewriter face, while others may be from any type of computer printer.

The quality of this reproduction is dependent upon the quality of the copy submitted. Broken or indistinct print, colored or poor quality illustrations and photographs, print bleedthrough, substandard margins, and improper alignment can adversely affect reproduction.

In the unlikely event that the author did not send UMI a complete manuscript and there are missing pages, these will be noted. Also, if unauthorized copyright material had to be removed, a note will indicate the deletion.

Oversize materials (e.g., maps, drawings, charts) are reproduced by sectioning the original, beginning at the upper left-hand corner and continuing from left to right in equal sections with small overlaps. Each original is also photographed in one exposure and is included in reduced form at the back of the book.

Photographs included in the original manuscript have been reproduced xerographically in this copy. Higher quality 6" $\times 9$ " black and white photographic prints are available for any photographs or illustrations appearing in this copy for an additional charge. Contact UMI directly to order.

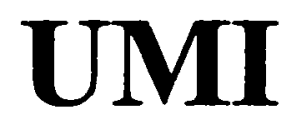

A Bell \& Howell Information Company 



\author{
A Thesis \\ Presented to \\ The Faculty of the Dapartment of Biological Sciences \\ San Jose State University
}

In Partial Fulfillment

of the Requirements for the Degree

Master of Science

By

Sujata Naidu

December, 1996 
OMI Number: 1382599

UMI Microform 1382599

Copyright 1997, by UMI Company. All rights reserved.

This microform edition is protected against unauthorized copying under Title 17, United States Code.

\section{UMI \\ 300 North Zeeb Road \\ Ann Arbor, MI 48103}


APPROVED FOR THE

DEPARTMENT OF BIOLOGICAL SCIENCES

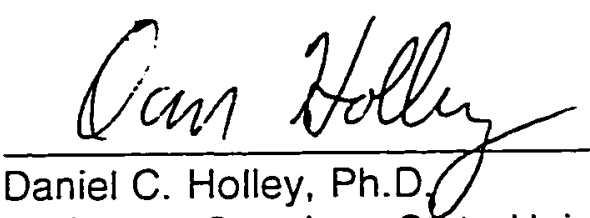

Professor, San Jose State University
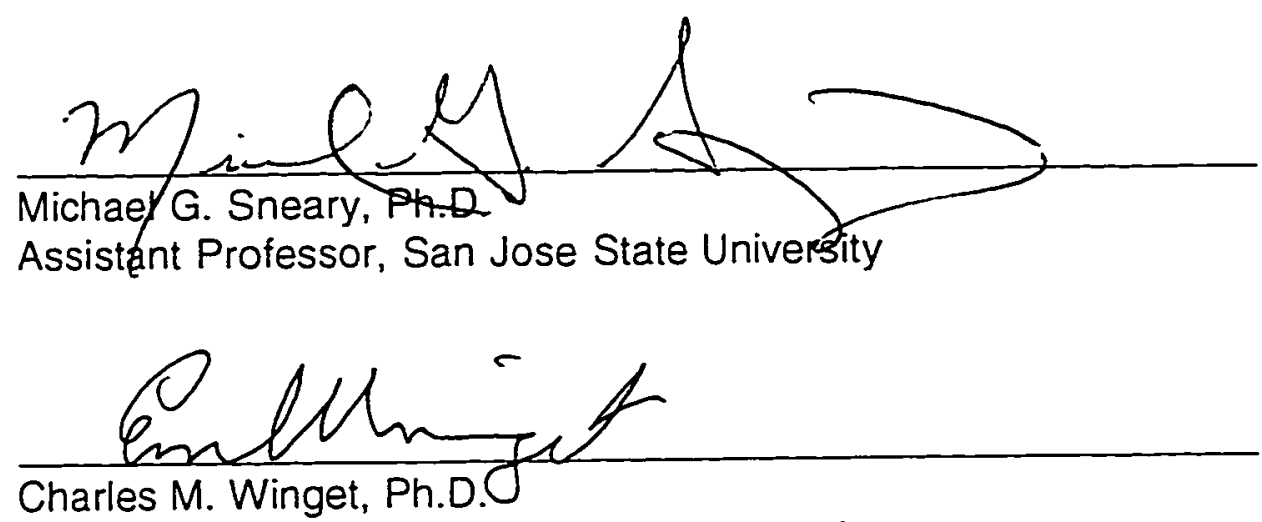

Payloads Scientist, NASA-Ames Research Center

APPROVED FOR THE UNIVERSITY

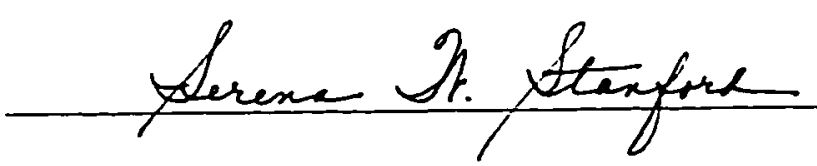


(C) 1996

Sujata Naidu

ALL RIGHTS RESERVED 


\section{ABSTRACT \\ EFFECTS OF HOUSING DENSITY ON MOUSE PHYSIOLOGY AND BEHAVIOR IN NASA ANIMAL ENCLOSURE MODULE SIMULATORS by Sujata Naidu}

This study was part of a larger project to qualify the NASA Animal Enclosure Module (AEM) for use with female mice. Goals for this investigation were to determine the optimum number of adult mice that could be housed in the $A E M$, to verify that the exhaust filter would contain mouse odors, determine if water and food access were adequate, and to verify normal animal growth and behavior.

Three population densities of mice were evaluated: 8,12 and 16 . The results of the first experiment were confounded due to increased ammonia in some of the cages because fallen food bars accumulated urine and impeded ventilation. A second experiment was, therefore, performed taking precautions to prevent fallen food bars and ammonia build-up.

These findings indicate that mice housed in AEM simulators for 30 days at densities of 8,12 or 16 are similar in terms of their growth, physiology and behavior. 


\section{ACKNOWLEDGEMENTS}

I would like to thank Dr. Daniel C. Holley for his guidance, support and friendship during the course of my graduate work. I have made a great deal of progress in my personal development under his tutelage. I would also like to thank the other members of my thesis committee, Dr. Charles Winget of NASAAmes Research Center and Dr. Michael Sneary of the Biology Department. Dr. Winget gave me the opportunity to conduct this study and has helped to guide me through every stage of this project while Dr. Sneary has been supportive and encouraging and a sheer pleasure to work with.

Gary Mele, my fellow graduate student and friend, was of invaluable assistance. I relied heavily on his technical expertise, advice on statistical analyses, and his quiet wisdom to help me through the periods I was conducting the studies and through the time I was writing my thesis..

I'd like to extend my gratitude to Dr. William Murray who helped me to learn some very useful techniques in Hematology that were required for this project. I would also like to acknowledge the excellent support of the following San Jose State University students: Andrew Bitmansour, Arlene Bitmansour, John Gossett, Edwin Hagnazari, Debra Hayes, Pauline Hormozian, Heather Morando, Patricia Rines, Adam Seddiqui, and Kirsten Smith. I would like to thank Dr. Ken Nashita (Chair of the Department of Psychology, San Jose State University) for helping us develop the behavioral scoring method for the Stress Assessment Battery.

Finally, l'd like to thank Dr. Lee Dorosz for always being there for me. 
TABLE OF CONTENTS

Acknowledgments

Table of Contents

List of Figures

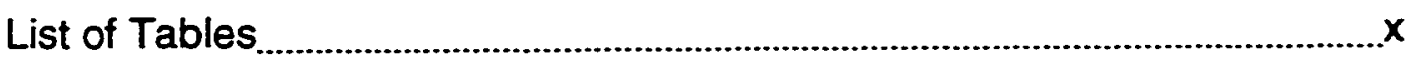

Introduction:

A. Background and Significance of the Study...............................1

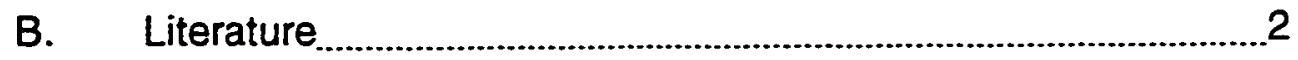

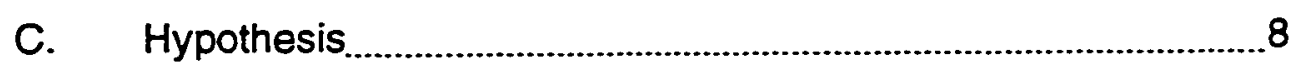

Materials and Methods:

A. Stress Assessment Battery Validation ........................................... 9

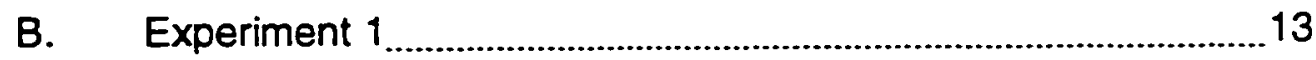

C. Experiment 2............................................................................. 15

D. Data Analysis .............................................................................. 18

Results:

A. Stress Assessment Battery Validation ......................................20

B. Experiment 1 ................................................................................. 32

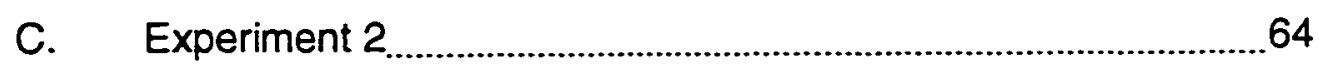

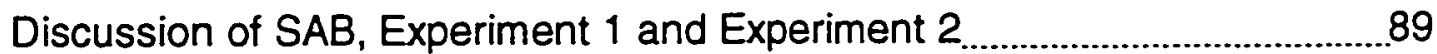

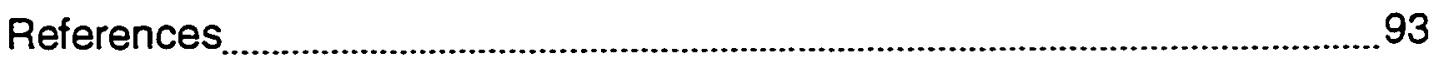




\section{LIST OF FIGURES}

Figure 1 (Stress Assessment Battery, SAB). Mean \pm S.E.M. food consumption per mouse per day of the stressed and control mouse groups.

Figure 2 (SAB). Mean \pm S.E.M. water consumption per mouse per day of the stressed and control mouse groups.

Figure 3 (SAB). Mean \pm S.E.M. body weight of the stressed and control mouse groups.

Figure 4 (SAB). Mean \pm S.E.M. weight gain and weight gain as a percentage of start weight.

Figure 5 (SAB). Mean \pm S.E.M. heart weight and heart weight as a percentage of final body weight.

Figure 6 (SAB). Mean \pm S.E.M. kidney weight and kidney weight as a percentage of final body weight.

Figure 7 (SAB). Mean \pm S.E.M. adrenal weight and adrenal weight as a percentage of final body weight.

Figure 8 (SAB). Mean \pm S.E.M. spleen weight and spleen weight as a percentage of final body weight.

Figure 9 (SAB). Mean \pm S.E.M. thymus weight and thymus weight as a percentage of final body weight. 28

Figure 10 (SAB). Plasma corticosterone mean \pm S.E.M. in the stressed and control groups.

Figure 11 (SAB). Plasma protein mean \pm S.E.M. 30

Figure 12 (Experiment 1). Mean \pm S.E.M. daily water consumption per cage and per mouse.

Figure 13 (Experiment 1). Mean \pm S.E.M. starting body weight (day 1) and mean \pm S.E.M. ending body weight (day 30 ) 38

Figure 14 (Experiment 1). Mean \pm S.E.M. body weight gain and mean \pm S.E.M. per cent body weight gain. 
Figure 15 (Experiment 1). Mean \pm S.E.M. heart weight and mean \pm S.E.M. heart weight as a percentage of final body weight.

Figure 16 (Experiment 1). Mean \pm S.E.M. kidney weight and mean \pm S.E.M. kidney weight as a percentage of final body weight.

Figure 17 (Experiment 1). Mean \pm S.E.M. bilateral adrenal weight and mean \pm S.E.M. bilateral adrenal weight as a percentage of final body weight. 47

Figure 18 (Experiment 1). Mean \pm S.E.M. spleen weight and mean \pm S.E.M. spleen weight as a percentage of final body weight.

Figure 19 (Experiment 1). Mean \pm S.E.M. thymus weight and mean \pm S.E.M. thymus weight as a percentage of final body weight.

Figure 20 (Experiment 1). Mean \pm S.E.M. plasma corticosterone and mean \pm S.E.M. plasma protein.

Figure 21 (Experiment 1). Mean \pm S.E.M. total leukocyte count and mean \pm S.E.M. eosinophil count.

Figure 22 (Experiment 1). Mean \pm S.E.M. basophil count and mean \pm S.E.M. neutrophil count.

Figure 23 (Experiment 1). Mean \pm S.E.M. total monocyte count and mean \pm S.E.M. lymphocyte count.

Figure 24 (Experiment 1). Mean \pm S.E.M. neutrophil to lymphocyte ratio.

Figure 25 (Experiment 2). Daily water consumption per mouse for each cage.

Figure 26 (Experiment 2). Mean \pm S.E.M. starting body weight (day 1 ) and mean \pm S.E.M. ending body weight (day 30 ).

Figure 27 (Experiment 2). Mean \pm S.E.M. body weight gain and mean \pm S.E.M. per cent body weight gain. 
Figure 28 (Experiment 2). Mean \pm S.E.M. heart weight and mean \pm S.E.M. heart weight as a percentage of final body weight.

Figure 29 (Experiment 2). Mean \pm S.E.M. kidney weight and mean \pm S.E.M. kidney weight as a percentage of final body weight.

Figure 30 (Experiment 2). Mean \pm S.E.M. bilateral adrenal weight and mean \pm S.E.M. bilateral adrenal weight as a percentage of final body weight.

Figure 31 (Experiment 2). Mean \pm S.E.M. spleen weight and mean \pm S.E.M. spleen weight as a percentage of final body weight.

Figure 32 (Experiment 2). Mean \pm S.E.M. thymus weight and mean \pm S.E.M. thymus weight as a percentage of final body weight.

Figure 33 (Experiment 2). Mean \pm S.E.M. plasma corticosterone and mean \pm S.E.M. plasma protein.

Figure 34 (Experiment 2). Mean \pm S.E.M. total leukocyte count and mean \pm S.E.M. eosinophil count.

Figure 35 (Experiment 2). Mean \pm S.E.M. neutrophil count and mean \pm S.E.M. monocyte count.

Figure 36 (Experiment 2). Mean \pm S.E.M. total lymphocyte count and mean \pm S.E.M. neutrophil to lymphocyte ratio. 


\section{LIST OF TABLES}

Table 1 (Experiment 1). Mouse cage type and number, mouse ID numbers, housing location, filter type, food type, and water delivery method.

Table 2 (Experiment 2). Mouse cage type and number, mouse ID numbers, housing location, filter type, food type, and water delivery method.

Table 3 (Stress Assessment Battery, SAB). Video scoring data used for behavioral evaluation.

Table 4 (SAB). Daily food consumption (grams). 21

Table 5 (SAB). Daily water consumption (ml). 21

Table 6 (SAB). Mouse initial (day 0 ) and final (day 14) body weight, weight gain and \% weight gain.

Table 7 (SAB). Organ weights in grams and as a percentage of final body weight (BW) of the stressed group.

Table 8 (SAB). Organ weights in grams and as a percentage of final body weight (BW) of the control group.

Table 9 (SAB). Plasma corticosterone ( $\mu \mathrm{g} / \mathrm{dl})$, plasma protein, and total leukocyte count in the stressed and control groups.

Table 10 (SAB). Differential WBC count, total eosinophil count and neutrophil to lymphocyte ratio of the stress group.

Table 11 (SAB). Differential WBC count, total eosinophil count and neutrophil to lymphocyte ratio of the stress group.

Table 12 (Experiment 1). Daily total and per mouse water consumption for each cage.

Table 13 (Experiment 1). Total food use (grams) over 30 days.

Table 14 (Experiment 1). Mouse initial (day 0) and final (day 30) body weight, weight gain in grams and \% weight gain. 
Table 15 (Experiment 1). Cage mean \pm S.E.M. for initial (day 0 ) and final (day 30) body weight, weight gain in grams, and $\%$ weight gain.

Table 16 (Experiment 1). Organ weights in grams and as a percentage of body weight (BW).

Table 17 (Experiment 1). Cage mean \pm S.E.M. for organ weights in grams and as a percentage of body weight (BW).

Table 18 (Experiment 1). Total white blood cell count (WBC), plasma corticosterone, plasma protein, eosinophil (EOS) count, basophil (BAS) count, neutrophil (NEU) count , monocyte (MON) count, lymphocyte (LYM) count, and neutrophil to lymphocyte ratio (N/L).

Table 19 (Experiment 1). Mean \pm S.E.M. white blood cell count (WBC), plasma corticosterone, plasma protein, eosinophil (EOS) count, basophil (BAS) count, neutrophil (NEU) count , monocyte (MON) count, lymphocyte (LYM) count, and neutrophil to lymphocyte ratio $(N / L)$.

Table 20 (Experiment 1). Video scoring data used for behavioral evaluation.

Table 21 (Experiment 1). Cage temperatures $\left({ }^{\circ} \mathrm{C}\right)$ for each test enclosure and the vivarium room temperature at the time of animal maintenance.

Table 22 (Experiment 1). Results of statistical testing for the effects of the number of mice per cage and the filter type used. 62

Table 23 (Experiment 1). Results of statistical testing for the effects of the cage type used. 63

Table 24 (Experiment 2). Daily total and per mouse water consumption for each cage.

Table 25 (Experiment 2). Food consumption (grams) over 30 days. 66

Table 26 (Experiment 2). Mouse initial (day 0) and final (day 30) body weight, weight gain in grams, and \% weight gain. 66 
Table 27 (Experiment 2). Cage mean \pm S.E.M. for initial (day 0 ) and final (day 30 ) body weight, weight gain in grams, and $\%$ weight gain.

Table 28 (Experiment 2). Organ weight in grams and as a percentage of final body weight (BW).

Table 29 (Experiment 2). Mean \pm S.E.M. organ weights by cage......73

Table 30 (Experiment 2). Total white blood cell count (WBC), plasma corticosterone, plasma protein, eosinophil (EOS) count, basophil (BAS) count, neutrophil (NEU) count , monocyte (MON) count, lymphocyte (LYM) count, and neutrophil to lymphocyte ratio (N/L).

Table 31 (Experiment 2). Mean \pm S.E.M. white blood cell count (WBC), plasma corticosterone, plasma protein, eosinophil (EOS) count, basophil (BAS) count, neutrophil (NEU) count , monocyte (MON) count, lymphocyte (LYM) count, and neutrophil to lymphocyte ratio (N/L).

Table 32 (Experiment 2). Video scoring data used for behavioral evaluation.

Table 33 (Experiment 2). Adjusted $G$ for ANOVA 1 from the test for normally distributed data and Burr-Foster q-test results for homogeneity of variances.

Table 34 (Experiment 2). Adjusted $G$ for ANOVA 1 from the test for normally distributed data and Burr-Foster q-test results for homogeneity of variances. 


\section{INTRODUCTION:}

\section{A. Background and Significance of the Study}

This project was part of the effort to qualify the NASA Animal Enclosure Module (AEM) for use with female mice. Goals for the investigation were to determine the optimum number of adult mice that could be housed in the AEM, to verify that the exhaust filter would contain mouse odors, determine if water and food access were adequate, and to verify normal animal growth and behavior.

In order to determine if health and well-being of the mice were compromised by the AEM, the Stress Assessment Battery (S.A.B.) was developed. It was verified using immobilization as a stressor.

Three different population densities of mice were evaluated: 8,12 and 16 . The project consisted of two phases. The results of the first phase experiment were confounded due to increased ammonia in some of the cages because fallen food bars accumulated urine and impeded ventilation. A second phase experiment was, therefore, performed taking precautions to prevent fallen food bars and ammonia buildup. 


\section{B. Literature}

\section{Animal Stress: Historical Perspective}

In the 1930's, Hans Selye (1936) developed a comprehensive theory of stress and described biological correlates associated with it. He suggested that a variety of stressors (e.g., pain, toxins, cold, heat, or starvation) when exposed to an animal chronically would result in physiological changes that may, in turn, develop into a pathological state. In his stress research, Selye repeatedly found an adrenal-cortical response. This led to the development of his General Adaptation Syndrome (GAS). GAS refers to a nonspecific response to stress and consists of three stages: (1) an immediate alarm reaction, (2) a resistance stage, and eventually (3) exhaustion (Selye, 1950).

The initial alarm reaction to an external stressor activates the sympathoadrenal system resulting in elevated levels of catecholamines. This reaction was described by Walter Canon (1929) as the "fight-flight" response whereby the animal prepares to escape or struggle against the nocuous stimulus. The modern view, as summarized by Tepperman and Tepperman (1987) is that an immediate defense against volume depletion and hypotension is brought about by: a) an increase in autonomic sympathetic tone resulting in sympathoadrenal medullary activation thus increasing systemic catecholamines, b) activation of the hypothalamic-anterior pituitaryadrenocortical axis resulting in production of ACTH (adrenocorticotrophic hormone) and glucocorticosteroids, $c$ ) activation of the hypothalamic-posterior pituitary axis thus releasing ADH (anti-diuretic hormone), and d) autonomic activation of the reninangiotensin pathway.

Selye's resistance stage was characterized by the release of adrenal glucocorticoids. The actions of glucocorticoids are slower than the rapid actions of catecholamines, but according to Selye, they provide the sustained secondary 
response (resistance). The modern view is that the various physiological mechanisms respond to the insult (stress) to homeostatically maintain the dynamic conditions of the internal environment. From a metabolic stand point, this occurs in two phases. First, the insulin-glucagon ratio shifts such that the insulin secretion decreases and the glucagon secretion increases. This, along with the increased glucocorticoid levels, results in a metabolic profile that includes proteolysis, glycogenolysis, gluconeogenesis and lipolysis. In the next phase, the insulin glucagon ratio reverses, growth hormone and other mitogenic factors increase in concentration thus resulting in the reparative process such as wound healing, scar formation, antibody formation, hematopoiesis, etc.

When the animal is exposed to prolonged periods of stress, it eventually enters the third GAS stage, exhaustion. The modern view holds that if the physiological mechanisms can not maintain homeostasis then pathology may result. Prolonged high levels of glucocorticoids lead to a number of physiological changes including hypertrophy of the adrenal gland, suppression of the immune system, gastrointestinal ulceration and muscle atrophy.

Selye's early model failed to predict that hypersecretion of glucocorticosteroids would inhibit many systems including the immune system, mechanisms associated with inflammation and wound healing, mechanisms associated with extracellular fluid volume control and hormonal mechanisms associated with regulation of metabolism. Munck et al. (1984) suggest that glucocorticosteroids are permissive or "synergistic" with many hormones, prostaglandins, proteases, lymphokines and other "cellular mediators" in promoting full development and function of physiological systems and mechanisms involved in homeostasis and defence against disease and injury. During prolonged "chronic" stress situations, increased glucocorticosteroid secretion is not for protection from the source of the stress itself, but rather for protection against the effects of prolonged hyperactivity (overshoot) of the body's normal physiological 
mechanisms that respond to stress. In other words, chronic overactivity of some mechanisms may be maladaptive and in themselves produce damage or be a threat to homeostasis. Immunosuppression would thus be an example of this adaptive mechanism, so that overactivity of the immune system might not damage endogenous tissues.

\section{Effects of Chronic Stress}

Elevated plasma corticosterone is still generally accepted as an indicator of stress. However, these levels fluctuate with the circadian rhythm of the animal (i.e., the plasma corticosterone concentration peaks just prior to activity onset). Another complication when evaluating plasma corticosterone is the time taken to withdraw a blood sample from the moment an animals' cage is disturbed. Any disturbance to the cage alarms the animal thus causing an acute adrenal activation. Plasma corticosterone concentrations in blood samples withdrawn within three minutes should not be greatly affected by this acute response ( $G$. Moberg, personal communication). We therefore took precautions to account for our sample time. Most samples were withdrawn within three minutes from the time the cage was lifted from its chamber. Blood samples withdrawn after three minutes were considered questionable for determination of undisturbed plasma corticosterone concentrations. Because of the problems with sample time and circadian changes, adrenal gland weights may be a better indicator of chronic stress.

Chronic high plasma corticosterone is reflected by adrenal gland hypertrophy (Daniels-Severs, 1973) and leads to immunosuppression. We expected to see increased adrenal gland weights, decreased thymus and spleen weights, decreased total white blood cell counts (leukopenia) and a change in the leukocyte profile namely eosinopenia, and neutrophilia (which would be reflected in neutrophil to lymphocyte ratio) (Jain, 1986). 
The immunosuppressive effect caused by chronic elevation of plasma corticosterone has recently been re-investigated. Garvy, et al. (1993), ascertained that elevated plasma corticosterone can cause a marked reduction in the number of cycling $B$ cells in murine bone marrow and induce apoptosis. It has also been demonstrated that the stress-induced corticosterone response down-regulates mononuclear cell trafficking (Herman, et al., 1995)

There are now studies which indicate that another serum factor may be also responsible for the supressed lymphocyte proliferation. Preliminary exaination of this factor indicates that it is a large molecular weight protein that is stable to heat, temperature, organic solvents and proteases (Zha, et al., 1992). The serum factor is yet to be identified but has been shown by Zha, et al. to suppress lymphocyte proliferation when its production is induced by restraint stress in rodents.

Total plasma protein concentration is affected by a number of factors. For example, hormonal mechanisms, diet, hemoconcentration, pregnancy, lactation and age all play a role in influencing plasma protein concentrations. In our study, mice were of the same age and sex and were given the same diet. Hence, factors affecting the plasma protein concentration here are limited to hormonal control, stress related factors and hemoconcentration (e.g., dehydration). Insulin, growth hormone, estrogens and androgens are anabolic and act to facilitate protein synthesis and diminish nitrogen excretion. Thyroid hormones are anabolic at normal levels but catabolic at high doses. Glucocorticoids are catabolic and utilize amino acids for gluconeogenesis (via increased proteolysis).

It is difficult to conclude that a change in plasma protein is due to some hormonal change or to the level of hydration of the animal or to some combination of both factors. Nevertheless, it would be an indicator of a difference in physiological state of a test animal when compared to a control. 
Lastly, under conditions of extreme prolonged stress, it is common to find stomach ulcerations due to chronic elevated glucocorticosteroid secretion. This mechanism is associated with a decrease in prostaglandins (PGE) and an increased hydrochloric acid secretion.

\section{Crowding Stress in Mice}

Historically, the concept of a nonspecific response to stress was generally accepted by physiologists. However, it should be noted that the actual hormonal response pattern and the various physiological changes differ among animal species, between strains within a species and from one type of stressor to another. It also affects male and female mice differently (Svec, et al., 1989).

"Crowding" has been defined as an increase in the intensity of competition for space (Christian, 1963). Social stress leading to increased aggressiveness occurs because mice form social dominance heirarchies when in groups. Fighting amongst mice, being a relatively natural form of physiological stress, has been used as a tool for studying normal mechanisms of neuro-endocrine adaptation and control. Fighting amongst male mice, in particular, has resulted in enlarged adrenals, spleens and hearts as well as marked increases in brain and adrenal catecholamines (Welch and Welch, 1969). Fighting also affects the immune system in dominant and submissive mice in a different manner than psychological or mild aggressive encounters (Hardy, et al., 1990). Hardy, et al. found that submissive mice had lower T-cell proliferation than dominant mice suggesting that a stronger stress response is elicited from submissive mice.

The physiological, socio-behavioral and morphological effects of overpopulation have been studied for many years. Increases in population size induce a number of endocrine responses. Besides increased pituitary-adrenocortical activity, there is decreased reproductive function (Christian, 1964, Edwards and Dean, 1977, and 
Breazile, 1987). Factors implicated include rank of the females in the social heirarchy, fetal resorption, inhibition of estrus and sudden increases in infant mortality (Lloyd and Christian, 1969). Diverse stressors impede female receptivity and fertility. Epinephrine has been shown to disrupt lordosis behavior (thereby eliminating receptivity) but has not been shown to have an effect on implantation after insemination (deCatanzaro and Graham, 1992). Maternal stress has also been shown to induce a syndrome of fetal anomalies including encephalocele, supernumerary ribs, fused ribs and vertebral anomalies (Miller and Chernoff, 1995).

In summary, the effects of stress, particularly prolonged stress, have profound effects on mice which vary amongst strains, within a hierarchy in a strain and between the sexes (Griffiths and Anisman, 1994). The response to stress, while non-specific, has a profile that also varies between strains, within strains and between sexes and the type of stressor introduced to the mice. This study will focus on the crowding stressor using female Swiss Webster mice. 


\section{Hypothesis}

Null Hypothesis: The health and well-being of mice housed in the AEM are not compromised given population densities of 8,12 and 16 .

Based on the premise that a nocuous stressor elicits a non-specific response, an assessment battery of measures was developed. The assessment battery of tests (Stress Assessment Battery) includes:

1) General appearance of coat, nose, eyes and ears

2) Behavioral assessment

3) Food and water consumption

4) Body weight changes

5) Thymus, adrenal, spleen, heart and kidney weights

6) Plasma corticosterone concentration

7) Total plasma protein concentration

8) Total blood leukocyte count

9) Differential leukocyte count: neutrophil/lymphocyte ratio; eosinophil count

10) Gastric histology

Items for inclusion in the SAB were selected after consultation and review of the following:

1) report of NASA Workshop on Rodent Cage Sizing, March 30-31,1990;

2) personal communication with Dr. G.P. Moberg, University of California, Davis, editor of Animal Stress, American Physiological Society, 1985. 


\section{MATERIALS AND METHODS:}

\section{A. Stress Assessment Battery Validation}

1) Animals:

Twenty female Swiss-Webster mice, approximately $18 \mathrm{~g}$ (initial weight) were purchased from Simonsen Laboratories, Gilroy, CA. Ten became the experimental group that were exposed to daily immobilization stress (for fourteen days) and ten animals became the control group.

2) Food and Water:

Standard rodent laboratory chow (Teklad Sterilizable Rodent Diet, Harlan Teklad, Madison, WI) and distilled water were provided ad libitum.

3) Animal Housing:

Standard vivarium shoe-box cages $(27.5 \mathrm{~cm}$ length $X 17.2 \mathrm{~cm}$ width $X 12.3 \mathrm{~cm}$ height) lined with "Beta chip" bedding (Northeastern Product Corp., Warrensburg, NY; alder hardwood chips) held 5 mice each. The experimental group was housed in isolation chamber $\mathrm{DH} 542 \mathrm{~A}$ and the control group was housed in isolation chamber $\mathrm{DH}$ $542 \mathrm{C}$. Filter bonnets were placed on all cages. All mice were exposed to a $12 \mathrm{~L}: 12 \mathrm{D}$ light cycle with lights on at $0700 \mathrm{~h}$. Lighting was standard fluorescent. Light intensity was approximately $330-470$ lux

4) Restraint Method to Induce Stress Response:

Each animal from the experimental group was removed from its cage and placed in a plexiglass restraining device (catalogue identification number: 52-0460, Harvard Apparatus, South Natick, MA; $9.5 \mathrm{~cm} \times 2 \mathrm{~cm} \times 2 \mathrm{~cm}$ ) for three hours a day. The daily time chosen to restrain the animals was random between $1000-2100$. The animals could not turn around within the restrainer and the tail gate of the restrainer 
was adjusted to keep the animal contained without impairing its circulation. The mice were returned to their home cage immediately after the restraint period.

5) Daily measurements and health evaluation:

Water and food consumption were recorded daily from all rodent cages. A visual physical examination of the mice was done daily. Changes in the coat, eyes, nose and ears were taken as indicators of the physical well-being of the rodents.

Observation

Hair coat

Eyes

Nose

Ears

Tail

Behavioral

Postural
Acceptable

Smooth and glossy.

Clear, open, "bright"

No swelling

Clean, pink

Clean, Smooth

Exploring, grooming, playing

Head, back, tail

\section{Questionable}

Dull, rough, loose or stained hair.

Puffy, partly closed, pink rings, discharge, ulceration.

Discharge- clear yellowish pus, "bloody".

Scaly, dirty, yellowish.

Rough, scaly, stained

Fighting,

Arched back, head tilted

*Prepared by Dr. Sig Rich, Campus Veterinarian 


\section{6) Behavior Assessment:}

Mice were videotaped during the dark portion (mouse activity phase) of the light cycle using a Toshiba IK-M30M CCD camera system. Illumination came from one 15 watt Kodak darkroom lamp model A with a glass IR filter. A Magnavox VR9942 VHS videotape recorder recorded the camera video.

Videotapes were scored by observing 10 minutes per hour for the first two hours of each tape. The observer divided the cage volume into four quadrants. Each quadrant was scored independently by counting the number of times each mouse engaged in a particular type of activity over the 10 minute scoring period. Activity classes were defined as follows: 0 - sleeping, still, sniffing; 1 - eating, drinking; 2 grooming, moving; 3 - social interaction; 4 - aggressive behavior; 5 - running. We defined social behavior as chasing or wrestling and aggressive behavior as biting. Scores for all mice and scoring periods were added together to obtain one number per activity class for each tape.

\section{7) Euthanasia method:}

The animals were euthanized one cage at a time. The cage was removed quietly from the isolation chamber and the cage was taken into an adjoining room where it was placed in a large container (approximately 5 gallon volume) pre-saturated with $100 \%$ carbon dioxide. A timer was started immediately upon initial handling of the cage. The animals were kept in the carbon dioxide until they were unconscious (about one minute). Subsequently, each mouse was given to a member of the dissection team for blood withdrawal via heart puncture.

The blood was collected using heparinized $3 \mathrm{cc}$ syringes. An aliquot of the whole blood was immediately used to perform total white blood cell counts (Unopette microcollection system, Becton-Dickinson, Rutherford, New Jersey). Another aliquot was used to prepare slides for differential white blood cell counting. The remaining blood was centrifuged $\left(a^{\circ} 4^{\circ} \mathrm{C}\right.$ ) and the plasma immediately frozen for later analysis.

All blood samples were labelled with time from initial handling of cage to completion of blood withdrawal. Samples that took longer than 3 minutes to obtain 
were considered questionable for determination of undisturbed plasma corticosterone concentrations.

8) Dissection of mice:

The mice were weighed before they were dissected and their stomachs, hearts, spleens, kidneys, adrenals and thymus glands removed and weighed. The stomachs were gently washed with buffered $10 \%$ formalin and then stored in formalinfilled jars for later histopathological analysis by pathologists at CVD, Inc. (Sacramento, CA).

9) Plasma corticosterone :

Plasma corticosterone concentration was determined using an ImmunochemTM ${ }^{125}$ I Corticosterone RIA kit (catalogue number:07-120103, ICN Biomedicals, Inc., Irvine, CA). This double antibody radioimmunoassay is designed specifically for use with laboratory rats and mice.

10) Plasma protein:

Total plasma protein concentration was determined via the Lowry method using a diagnostic kit obtained from Sigma Chemical Co., St. Louis, MO, (Catalogue number 690A.)

11) Leukocyte analysis:

Total white blood cell count was determined using the Unopette method (Catalogue number 5853,Becton-Dickinson, Rutherford, New Jersey)). These counts were done within hours of the termination of the mice. Blood smears were stained with Diff-quik (Catalogue number: B4132-1,Scientific Products, McGaw Park,IL) and differential leukocyte counts were determined by Mr. Wayne Pinard, AHT, (Vetenary Lab Technician, Adobe Animal Hospital, Los Altos, CA).

12) Stomach histopathology:

The stomach tissue was trimmed, embedded, sectioned and examined by pathologists at CVD, inc. (Sacramento,CA). 


\section{B. Experiment 1: Mouse Density Study}

\section{Animals}

Eighty-eight female Swiss-Webster mice (Simonsen Laboratories, Gilroy, CA) were weighed $(21.8 \pm 1.0$ grams, mean \pm S.D. $)$ and ear punched to allow individual identification. The mice were placed in eight cages on experiment day 0 . Six NASA supplied animal enclosure module simulators (Test Enclosures), three with ALFCO \#3A outlet filters, three with ALFCO \#4 outlet filters, held two full food bar plates, a standard water box with four lixits, and 8,12, or 16 mice. These six cages were housed in $\mathrm{DH} 639 \mathrm{~A}$. One large shoe box type cage (Vivarium Shoe Box Control, $42.5 \mathrm{~cm}$ length $\times 21 \mathrm{~cm}$ width $\times 12.3 \mathrm{~cm}$ height) in the Animal Care Facility and one AEM wire cage within a lexan housing (Vivarium Control Enclosure) in the Animal Care Facility each held eight of the remaining mice. The Vivarium Shoe Box Control was supplied with Techlad rodent diet (W) (Harlan Sprague-Dawley, Madison, WI) and distilled water from a standard vivarium cage water bottle. The Vivarium Control Enclosure held two food bar plates with NASA food bars and an AEM water box with four lixits. Distilled water from a graduated cylinder supplied the lixits. The study ran for 30 days. On Day 30 , the animals were removed from the cages and the SAB conducted. Table 1 shows the combinations of cage type, number of mice, and filter type used.

Table 1 (Experiment 1). Mouse cage type and number, mouse ID numbers, housing location, filter type, food type, and water delivery method.

\begin{tabular}{lcccccc}
$\begin{array}{c}\text { Cage } \\
\text { Designator }\end{array}$ & Location & $\begin{array}{c}\text { Number } \\
\text { of mice }\end{array}$ & $\begin{array}{c}\text { Mouse ID } \\
\text { Numbers }\end{array}$ & $\begin{array}{c}\text { Filter } \\
\text { Type }\end{array}$ & $\begin{array}{c}\text { Food } \\
\text { Type }\end{array}$ & $\begin{array}{c}\text { Water } \\
\text { Delivery }\end{array}$ \\
\hline Test enclosure \#3 & DH639-A & 8 & $(\# 1-8)$ & ALFCO \#3A & bars & lixit \\
Test enclosure \#6 & DH639-A & 12 & $(\# 9-20)$ & ALFCO \#3A & bars & lixit \\
Test enclosure \#5 & DH639-A & 16 & $(\# 21-36)$ & ALFCO \#3A & bars & lixit \\
Test enclosure \#7 & DH639-A & 8 & $(\# 37-44)$ & ALFCO \#4 & bars & lixit \\
Test enclosure \#1 & DH639-A & 12 & $(\# 45-56$ & ALFCO \#4 & bars & lixit \\
Test enclosure \#2 & DH639-A & 16 & $(\# 57-72)$ & ALFCO \#4 & bars & lixit \\
Test enclosure \#4 & DH639-A & 0 & N/A & ALFCO \#4 & N/A & N/A \\
Vivarium Shoe Box Control & ACF vivarium & 8 & $(\# 73-80)$ & N/A & chow & bottle \\
Vivarium Control Enclosure & ACF vivarium & $\mathbf{8}$ & $(\# 81-88)$ & N/A & bars & lixit
\end{tabular}

ACF = University Animal Care Facility (DH741A) 


\section{Lighting}

All mice were exposed to a 12L:12D light cycle with lights on at 0700h. Light intensity was approximately $340-470$ lux just outside the Lexan housing of the test enclosures. Lighting was standard fluorescent.

\section{Test Enclosure Air Flow}

Air speed in the $4^{n}$ (internal diameter) PVC pipe attached to the test enclosures was set to 180 feet per minute on test day 0 . This was equivalent to 14.8 cubic feet per minute of air flow. Neither the Vivarium Shoe Box Control nor the Vivarium Control Enclosure had forced air flow. Air speed was measured daily with a TSI, Inc. Velocicalc Model 8350 hot wire air flow meter.

\section{Ammonia Measurement}

Ammonia concentration within cages was measured using Sensidyne $\otimes$ Gastec No. 3L ammonia analyzer tubes (\#32011-335, VWR Scientific, San Francisco, CA). A Sensidyne® Gastec pump (kit No. 800) drew either $100 \mathrm{ml}$ or $200 \mathrm{ml}$ of air through each analyzer tube. Test enclosure internal air was drawn from the upstream differential pressure gauge port located between the inner AEM cage and the outlet filter. Test enclosure outlet plenum air was drawn from the downstream differential pressure gauge port located in the wall of the outlet plenum (see Figure 1). Test enclosure internal ammonia concentration was measured on test days 15, 17, 21, 24, and 29. Test enclosure outlet plenum ammonia concentration was measured on test days $11,14,15,21,24$, and 29. 


\section{Experiment 2: Mouse Density Study}

\section{Animals}

Fifty-two female Swiss-Webster mice (Simonsen Laboratories, Gilroy, CA) were weighed $(28.6 \pm 0.3$ grams, mean $\pm S . E$.) and ear punched to allow individual identification. The mice were placed into five cages on experiment day 0 . Three NASA supplied animal enclosure module simulators (Test Enclosures), all with ALFCO \#3A outlet filters, held two full food bar plates, a standard water box with four lixits, and 8,12, or 16 mice. These cages were housed in DH 639A. One large shoe box type cage (Vivarium Shoe Box Control, $42.5 \mathrm{~cm}$ length $\times 21 \mathrm{~cm}$ width $\times 12.3 \mathrm{~cm}$ height) and one AEM wire cage within a lexan housing (Vivarium Control Enclosure) each held eight of the remaining mice in the Animal Care Facility (DH 741A). The Vivarium Shoe Box Control was supplied with Techlad Rodent Diet (W) (Harlan Sprague-L¿2wley, Madison, WI) and distilled water from a standard vivarium cage water bottle. The Vivarium Control Enclosure held two food bar plates with NASA food bars and an AEM water box with four lixits. Distilled water from a graduated cylinder supplied the lixits. The study ran for 30 days. On Day 30 , the animals were removed from the cages and the SAB conducted. Table 2 shows the combinations of cage type, number of mice, and filter type used.

Table 2 (Experiment 2). Mouse cage type and number, mouse ID numbers, housing location, filter type, food type, and water delivery method.

\begin{tabular}{lcccccc}
$\begin{array}{c}\text { Cage } \\
\text { Designator }\end{array}$ & Location & $\begin{array}{c}\text { Number } \\
\text { of mice }\end{array}$ & $\begin{array}{c}\text { Mouse ID } \\
\text { Numbers }\end{array}$ & $\begin{array}{c}\text { Filter } \\
\text { Type }\end{array}$ & $\begin{array}{c}\text { Food } \\
\text { Type }\end{array}$ & $\begin{array}{c}\text { Water } \\
\text { Delivery }\end{array}$ \\
\hline Test Enclosure \#2 & DH639-A & 8 & $(\# 1-8)$ & ALFCO \#3A & bars & lixit \\
Test Enclosure \#4 & DH639-A & 12 & $(\# 9-20)$ & ALFCO \#3A & bars & lixit \\
Test Enclosure \#1 & DH639-A & 16 & $(\# 21-36)$ & ALFCO \#3A & bars & lixit \\
Test Enclosure \#3 & DH639-A & 0 & N/A & ALFCO \#3A & N/A & N/A \\
Vivarium Control Enclosure & ACF vivarium & 8 & $(\# 37-44)$ & N/A & bars & lixit \\
Vivarium Shoe Box Control & ACF vivarium & 8 & $(\# 45-52)$ & N/A & chow & bottle
\end{tabular}

ACF $=$ University Animal Care Facility (DH741) 


\section{Test Enclosures}

\section{Lighting}

All mice were exposed to a $12 \mathrm{~L}: 12 \mathrm{D}$ light cycle with lights on at $0700 \mathrm{~h}$. Light intensity was approximately $330-470$ lux just outside the Lexan housing of the Test Enclosures. Lighting was standard fluorescent.

\section{Food Bar Plates}

Food bar plates were prepared by NASA personnel using flight stock AEM food bars manufactured 6/21/93. Food bars were placed vertically rather than horizontally with respect to the launch orientation, as specified in the standard food bar plate preparation procedure. Two filled food bar plates were placed in each cage at the ends of the cage. The water box had no food bar plates attached. One full food bar plate was added to the Test Enclosure \#1 (16 mice) water box, on test day 23.

\section{Air Speed Measurement}

Air speed in the $4^{\prime \prime}$ i.d. PVC pipe attached to each Test Enclosures was set to 180 feet per minute on test day 0 . This was equivalent to 14.8 cubic feet per minute of air flow. Neither the Vivarium Shoe Box Control nor the Vivarium Control Enclosure had forced air flow.

Air speed was measured daily with a TSI, Inc. Velocicalc Model 8350 hot wire air flow meter. Air flow in cubic feet per minute, cfm, was calculated using the air speed and inside diameter of the pipe. 


\section{Ammonia Measurement}

Ammonia concentration within the occupied cages and downstream from the outlet filter was measured using Sensidyne Gastec No. 3L ammonia analyzer tubes (\#32011-335, VWR Scientific, San Francisco, CA). A Sensidyne@ Gastec pump (kit No. 800) drew either $100 \mathrm{ml}$ or $200 \mathrm{ml}$ of air through each analyzer tube. Test Enclosure internal air was drawn from the upstream differential pressure gauge port. Test Enclosure outlet plenum air was drawn from the downstream differential pressure gauge port (see Figure 1). Test Enclosure ammonia concentration was measured every day. Vivarium Control Enclosure ammonia concentration was measured every other day starting with test day 2.

\section{Methylamine Measurement}

Methylamine concentrations within the occupied cages and downstream from the outlet filter were measured using Dräeger triethylamine analyzer tubes (number 5/A, Brenton Safety, Inc., South San Francisco, CA). An Accuro Delux pump drew either $100 \mathrm{ml}$ or $200 \mathrm{ml}$ of air through each analyzer tube. Test Enclosure internal air was drawn from the upstream differential pressure gauge port. Test Enclosure outlet plenum air was drawn from the downstream differential pressure gauge port (see Figure 1). Test Enclosure methylamine concentration was measured every day starting with test day 3. Vivarium Control Enclosure methylamine concentration was measured every other day starting with test day 4 . 


\section{Data Analysis}

\section{Data Analysis Overview}

Individual animal parameters comprise the bulk of the data collected from this study. Animal parameters included mouse body and organ weights and blood measurements. Data for each individual parameter were divided into two sets by cage number. One set of cages tested for effects due to the number of mice per cage. The other set tested for the effect of the type of cage used. Each cage set was checked for normality of distribution. Cage variances within a set were compared to test for homogeneity of variances. Sets which passed these checks were tested for differences using analysis of variance (ANOVA). Sets which did not pass the checks were tested for differences using the nonparametric Kruskall-Wallis test (Sokal and Rohlf, 1981).

Daily cage parameters, water and air flow, were also measured and recorded. Data for each daily cage parameter were divided into two sets of cages as for individual parameters. The sets of daily data were tested for differences among cages using a two way ANOVA without replication (Sokal and Rohlf, 1981).

\section{Data Analysis Details}

ANOVA requires the data in each cage to be normally distributed and the variances of cages within an ANOVA to be homogeneous. To test whether data conformed to the normal distribution, data for all mouse cages within each cage set were combined into one group after subtracting the cage mean from each cage value. We compared the frequency distribution of each combined group to the expected normal frequency distribution using the $G$ test (Sokal and Rohlf, 1981), a test similar to but more accurate than the chi square test. Data were considered to be normally distributed for $P<.05$. Variances of cage means for cages within each cage set were then checked for homogeneity using the Burr-Foster Q-test. Variances were 
homogeneous for $q<q_{\text {em[.01] }}$. A square root transform was applied to data which did not pass either the normality or the homogeneity test. These data were then reanalyzed. Data or transformed data which passed the normality and homogeneity of variances checks were tested using analysis of variance (ANOVA). Data which could not be transformed to correct normality or homogeneity problems were analyzed using the nonparametric Kruskal-Wallis test. Any parameter with a significant result in the ANOVA was also tested with the Student-Newman Keuls test (Glantz, 1992). This tested for significant differences between any two groups within the ANOVA for the characteristic which gave the significant ANOVA result.

Data for mice were divided, by cage, into two sets for analysis. Cage set one contained data from all three groups of mice in the Test Enclosures. Groups in this set differed in the number of mice per cage. One way ANOVAs were used to compare individual and daily parameters among these groups of mice. Any differences detected in measured parameters among these groups would be due to the number of mice per cage. Cage set two contained the Test Enclosure holding 8 mice, the Vivarium Control Enclosure ( 8 mice) and the Vivarium Shoe Box control $(8$ mice). This arrangement allowed mice in the two control cages to be compared to mice in the Test Enclosure. One way ANOVAs were used to compare individual and daily parameters among these groups. Differences detected by this test were due to cage type. 


\section{RESULTS:}

\section{A. Stress Assessment Battery Validation}

Table 3 (S.A.B.). Video scoring data used for behavioral evaluation. The observer scored ten minutes of video per hour for six hours. The scores represent the sum frequency of each activity that occurred during the scoring epoch in all quadrants.

\section{Behavioral Score Category}

\begin{tabular}{llllllll}
\hline Treatment & $\mathbf{0}$ & $\mathbf{1}$ & $\mathbf{2}$ & $\mathbf{3}$ & $\mathbf{4}$ & $\mathbf{5}$ & Total \\
\hline Stressed Score & 412 & 120 & 826 & 195 & 0 & 118 & 1671 \\
Stressed \% Score & $25 \%$ & $7 \%$ & $49 \%$ & $12 \%$ & $0 \%$ & $7 \%$ & \\
Control Score & 207 & 30 & 545 & 40 & 0 & 3 & 825 \\
Control \% Score & $25 \%$ & $4 \%$ & $66 \%$ & $5 \%$ & $0 \%$ & $0 \%$ &
\end{tabular}

Video Scoring Definitions

0 - sleeping, still, sniffing

1 - eating, drinking

2 - grooming, moving

3 - social interaction

4 - aggressive behavior

5 - running 
Table 4 (S.A.B.): Daily food consumption (grams).

\begin{tabular}{ccc}
$\begin{array}{c}\text { Day } \\
\#\end{array}$ & $\begin{array}{c}\text { Stressed } \\
\text { Group }\end{array}$ & $\begin{array}{c}\text { Control } \\
\text { Group }\end{array}$ \\
\hline 1 & 28.89 & 35.30 \\
2 & 37.20 & 44.68 \\
3 & 38.89 & 44.69 \\
4 & 31.50 & 37.65 \\
5 & 44.83 & 54.37 \\
6 & 32.16 & 34.65 \\
7 & 41.81 & 44.99 \\
8 & 33.08 & 30.13 \\
9 & 33.09 & 30.13 \\
10 & 39.28 & 33.61 \\
11 & 37.97 & 34.76 \\
12 & 41.84 & 41.48 \\
13 & 46.54 & 45.69 \\
\hline Mean & 37.47 & 39.46 \\
SEM & 5.45 & 7.12 \\
\hline
\end{tabular}

Table 5 (S.A.B.): Daily water consumption (ml).

\begin{tabular}{ccc}
$\begin{array}{c}\text { Day } \\
\#\end{array}$ & $\begin{array}{c}\text { Stressed } \\
\text { Group }\end{array}$ & $\begin{array}{c}\text { Control } \\
\text { Group }\end{array}$ \\
\hline 1 & 49.0 & 62.0 \\
2 & 52.0 & 65.0 \\
3 & 53.0 & 65.0 \\
4 & 50.0 & 52.0 \\
5 & 70.0 & 74.0 \\
6 & 52.0 & 49.0 \\
7 & 69.0 & 83.0 \\
8 & 47.0 & 45.0 \\
9 & 47.0 & 46.0 \\
10 & 51.0 & 55.0 \\
12 & 54.0 & 49.0 \\
13 & 73.0 & 69.0 \\
\hline Mean & 55.7 & 58.9 \\
SEM & 9.0 & 11.8 \\
\hline
\end{tabular}


Figure 1 (S.A.B.). Mean \pm S.E.M. food consumption per mouse per day of the stressed and control mouse groups.

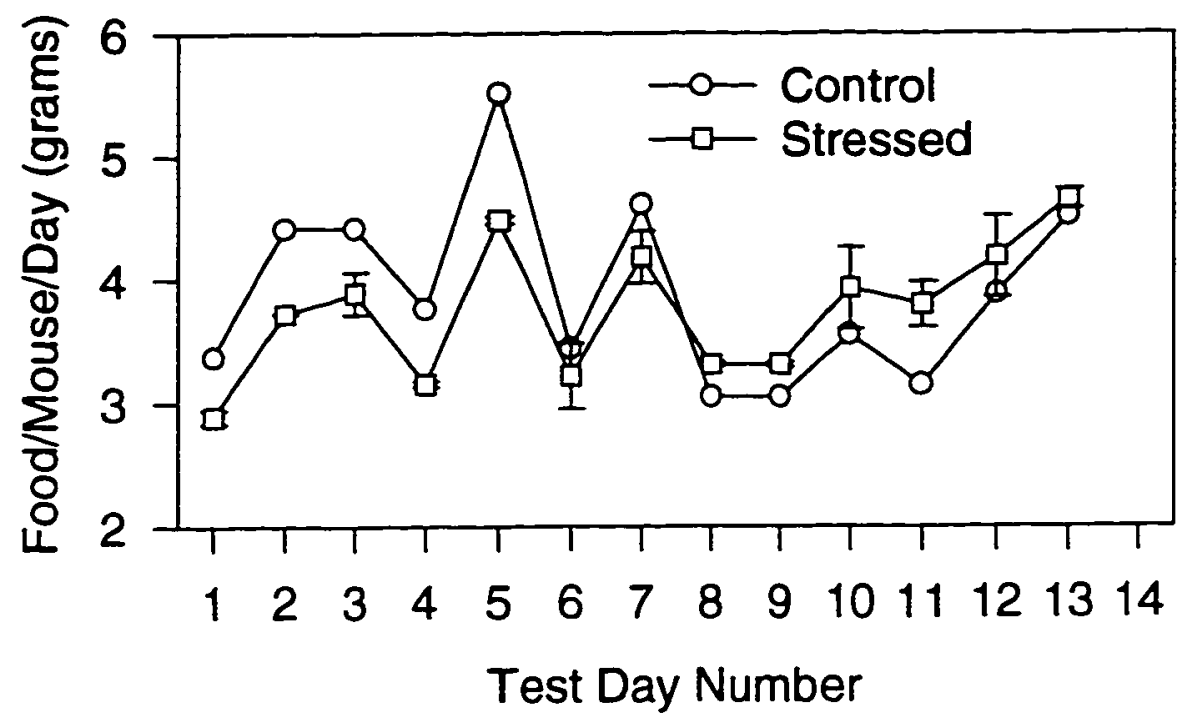

Figure 2 (S.A.B.). Mean \pm S.E.M. water consumption per mouse per day of the stressed and control mouse groups.

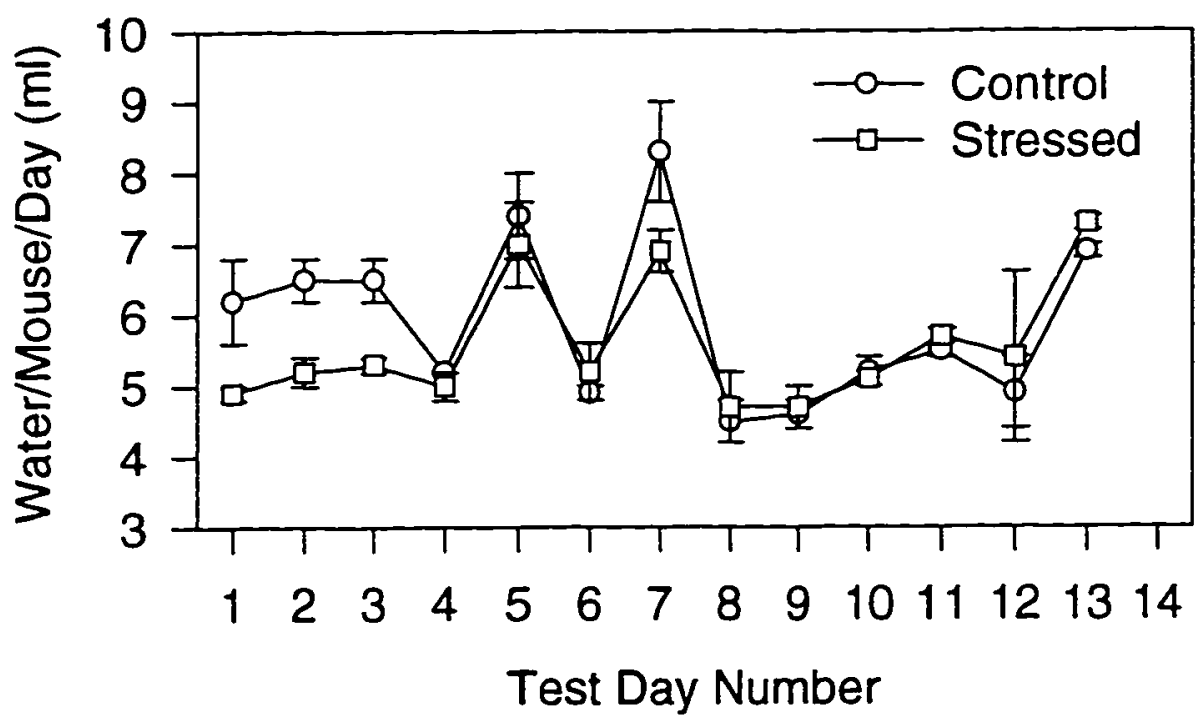


Table 6 (S.A.B.). Mouse initial (day 0) and final (day 14) body weight, weight gain in grams and \% weight gain.

\begin{tabular}{rllll}
$\begin{array}{c}\text { Mouse } \\
\#\end{array}$ & $\begin{array}{l}\text { Initial } \\
\text { Wt(g) }\end{array}$ & $\begin{array}{l}\text { Final } \\
\text { Wt(g) }\end{array}$ & $\begin{array}{l}\text { Gain } \\
(\mathrm{g})\end{array}$ & $\begin{array}{l}\text { Gain } \\
\text { (\%Initial) }\end{array}$ \\
\hline Stress 1 & 18.11 & 20.30 & 2.19 & 12.09 \\
Group 2 & 18.23 & 23.10 & 4.87 & 26.71 \\
3 & 19.46 & 22.90 & 3.44 & 17.67 \\
4 & 18.81 & 21.40 & 2.59 & 13.77 \\
5 & 17.57 & 20.20 & 2.63 & 14.67 \\
6 & 19.42 & 23.60 & 4.18 & 21.52 \\
7 & 18.41 & 21.00 & 2.59 & 14.07 \\
8 & 19.43 & 23.20 & 3.77 & 19.40 \\
9 & 18.77 & 22.40 & 3.63 & 19.34 \\
10 & 15.98 & 23.50 & 7.52 & 47.06 \\
\hline Mean & 18.42 & 22.20 & 3.74 & 20.66 \\
SEM & 1.06 & 0.42 & 0.50 & 3.24 \\
\hline
\end{tabular}

\begin{tabular}{cccll}
$\begin{array}{c}\text { Mouse } \\
\#\end{array}$ & $\begin{array}{l}\text { Initial } \\
\text { Wt(g) }\end{array}$ & $\begin{array}{l}\text { Final } \\
\text { Wt(g) }\end{array}$ & $\begin{array}{l}\text { Gain } \\
(\mathrm{g})\end{array}$ & $\begin{array}{l}\text { Gain } \\
\text { (\%Initial) }\end{array}$ \\
\hline Control11 & 17.76 & 24.20 & 6.44 & 36.26 \\
Group 12 & 17.60 & 25.60 & 8.00 & 45.46 \\
13 & 18.11 & 26.40 & 8.29 & 45.78 \\
14 & 17.45 & 23.90 & 6.45 & 39.96 \\
15 & 18.23 & 24.40 & 6.17 & 33.85 \\
16 & 18.61 & 23.10 & 4.49 & 24.13 \\
17 & 19.02 & 23.10 & 4.08 & 21.45 \\
18 & 19.12 & 25.10 & 5.98 & 31.28 \\
19 & 16.00 & 25.20 & 9.20 & 57.50 \\
20 & 18.11 & 24.90 & 6.79 & 37.49 \\
\hline Mean & 18.00 & 24.60 & 6.58 & 37.02 \\
SEM & 0.90 & 0.34 & 0.51 & 3.37 \\
\hline
\end{tabular}


Figure 3 (S.A.B.). Mean \pm S.E.M. body weight of the stressed and control mouse groups.

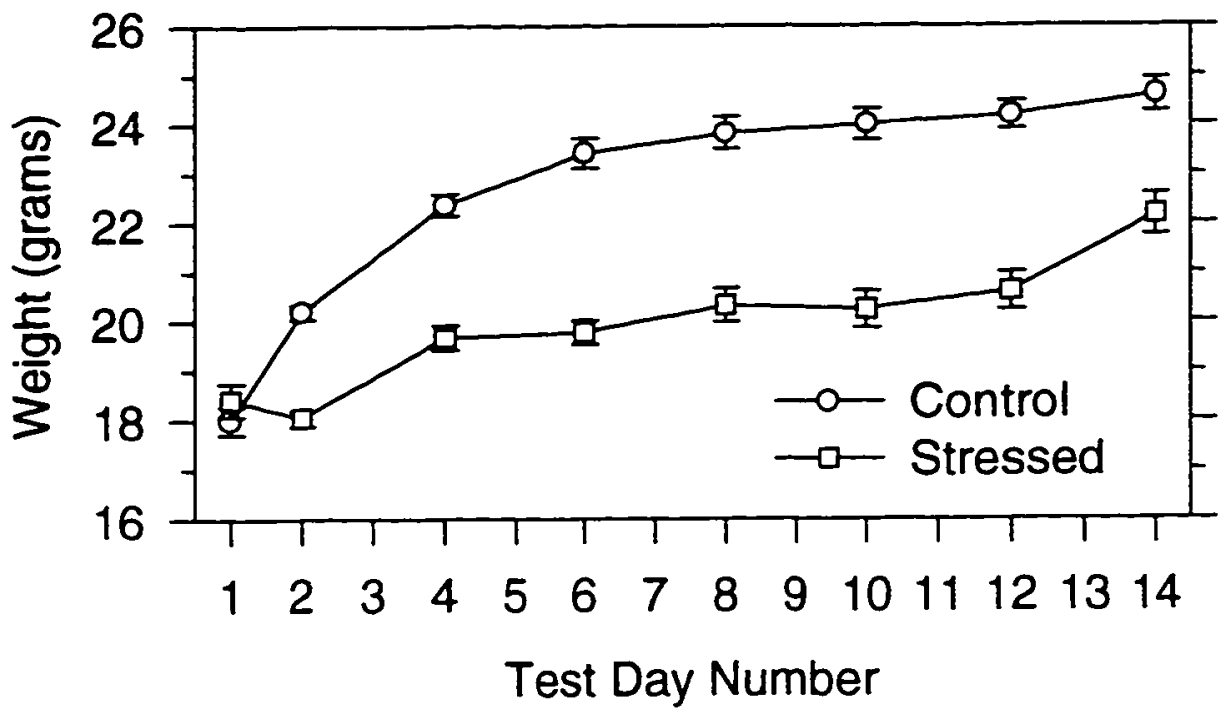

Figure 4 (S.A.B.). Mean \pm S.E.M. weight gain and weight gain as a percentage of start weight.
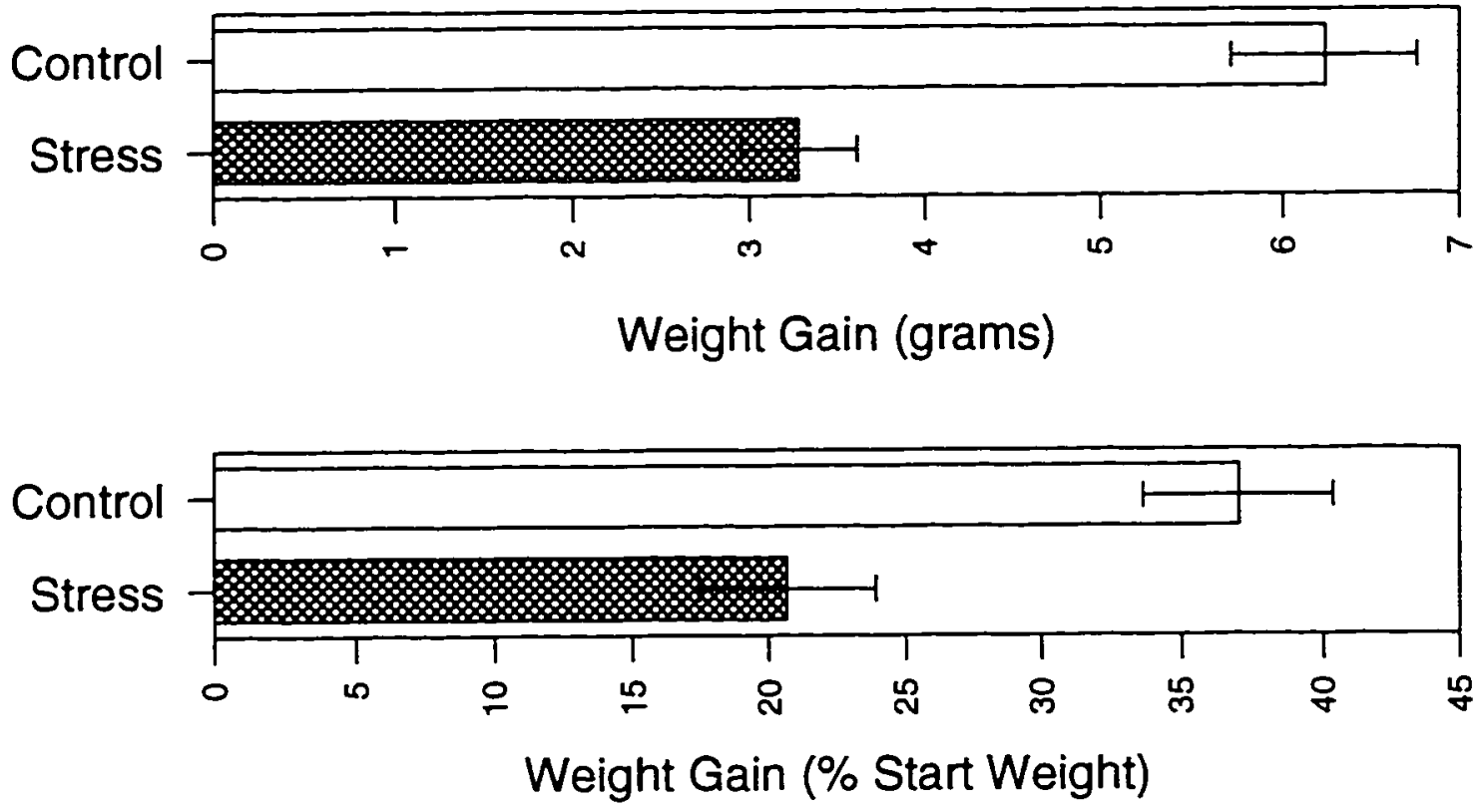
Table 7 (S.A.B.). Organ weights in grams and as a percentage of final body weight (BW) of the stressed group.

\begin{tabular}{cccccccccccc}
$\begin{array}{c}\text { Mouse } \\
\text { \# }\end{array}$ & $\begin{array}{c}\text { Body } \\
\text { W }(g)\end{array}$ & $\begin{array}{c}\text { Heart } \\
\text { Wt }(g)\end{array}$ & $\begin{array}{c}\text { Heart } \\
\% \text { BW }\end{array}$ & $\begin{array}{c}\text { Kidney } \\
\text { Wt }(g)\end{array}$ & $\begin{array}{c}\text { Kidney } \\
\% \text { BW }\end{array}$ & $\begin{array}{c}\text { Adrenal } \\
\text { Wt }(g)\end{array}$ & $\begin{array}{c}\text { Adrenal } \\
\% \text { BW }\end{array}$ & $\begin{array}{c}\text { Spleen } \\
\text { Wt }(g)\end{array}$ & $\begin{array}{c}\text { Spleen } \\
\% \text { BW }\end{array}$ & $\begin{array}{c}\text { Thymus } \\
\text { Wt }(g)\end{array}$ & $\begin{array}{c}\text { Thymus } \\
\% W\end{array}$ \\
\hline 1 & 20.3 & 0.094 & 0.463 & 0.281 & 1.384 & 0.0015 & 0.0074 & 0.056 & 0.276 & 0.066 & 0.325 \\
2 & 23.1 & 0.124 & 0.537 & 0.346 & 1.498 & 0.0013 & 0.0056 & 0.088 & 0.381 & 0.062 & 0.268 \\
3 & 22.9 & 0.097 & 0.424 & 0.301 & 1.314 & 0.0020 & 0.0087 & 0.042 & 0.183 & 0.042 & 0.183 \\
4 & 21.4 & 0.110 & 0.514 & 0.308 & 1.439 & 0.0018 & 0.0084 & 0.067 & 0.313 & 0.068 & 0.318 \\
5 & 20.2 & 0.099 & 0.490 & 0.265 & 1.312 & 0.0016 & 0.0079 & 0.045 & 0.223 & 0.062 & 0.307 \\
6 & 23.6 & 0.115 & 0.487 & 0.366 & 1.551 & 0.0018 & 0.0038 & 0.048 & 0.203 & 0.047 & 0.199 \\
7 & 21.0 & 0.096 & 0.457 & 0.303 & 1.443 & 0.0020 & 0.0095 & 0.042 & 0.200 & 0.060 & 0.286 \\
8 & 23.2 & 0.106 & 0.457 & 0.288 & 1.241 & 0.0019 & 0.0082 & 0.048 & 0.207 & 0.063 & 0.272 \\
9 & 22.4 & 0.090 & 0.402 & 0.289 & 1.290 & 0.0014 & 0.0063 & 0.064 & 0.286 & 0.086 & 0.384 \\
10 & 23.5 & 0.100 & 0.426 & 0.285 & 1.213 & 0.0029 & 0.0012 & 0.047 & 0.200 & 0.068 & 0.289 \\
\hline Mean & 22.2 & 0.103 & 0.466 & 0.303 & 1.369 & 0.0018 & 0.0082 & 0.055 & 0.247 & 0.062 & 0.283 \\
SEM & 0.42 & 0.003 & 0.013 & 0.0097 & 0.036 & 0.0001 & 0.0006 & 0.0046 & 0.020 & 0.0038 & 0.019 \\
\hline
\end{tabular}

Table 8 (S.A.B.). Organ weights in grams and as a percentage of final body weight (BW) of the control group.

\begin{tabular}{cccccccccccc}
$\begin{array}{c}\text { Mouse } \\
\#\end{array}$ & $\begin{array}{c}\text { Body } \\
\text { Wt }(\mathrm{g})\end{array}$ & $\begin{array}{c}\text { Heart } \\
\text { Wt }(\mathrm{g})\end{array}$ & $\begin{array}{c}\text { Heart } \\
\% \mathrm{BW}\end{array}$ & $\begin{array}{c}\text { Kidney } \\
\text { Wt }(\mathrm{g})\end{array}$ & $\begin{array}{c}\text { Kidney } \\
\% \mathrm{BW}\end{array}$ & $\begin{array}{c}\text { Adrenal } \\
\text { Wt }(\mathrm{g})\end{array}$ & $\begin{array}{c}\text { Adrenal } \\
\% \mathrm{BW}\end{array}$ & $\begin{array}{c}\text { Spleen } \\
\text { Wt }(\mathrm{g})\end{array}$ & $\begin{array}{c}\text { Spleen } \\
\% \mathrm{BW}\end{array}$ & $\begin{array}{c}\text { Thymus } \\
\text { Wt }(\mathrm{g})\end{array}$ & $\begin{array}{c}\text { Thymus } \\
\% \mathrm{BW}\end{array}$ \\
\hline 11 & 24.2 & 0.108 & 0.446 & 0.300 & 1.240 & 0.0013 & 0.0054 & 0.060 & 0.248 & 0.048 & 0.198 \\
12 & 25.6 & 0.119 & 0.465 & 0.253 & 0.988 & 0.0028 & 0.0109 & 0.085 & 0.332 & 0.099 & 0.387 \\
13 & 26.4 & 0.100 & 0.379 & 0.376 & 1.424 & 0.0015 & 0.0057 & 0.069 & 0.261 & 0.123 & 0.466 \\
14 & 23.9 & 0.109 & 0.456 & 0.342 & 1.431 & 0.0023 & 0.0096 & 0.077 & 0.322 & 0.106 & 0.444 \\
15 & 24.4 & 0.113 & 0.463 & 0.242 & 0.992 & & & 0.080 & 0.328 & 0.089 & 0.365 \\
16 & 23.1 & 0.088 & 0.381 & 0.258 & 1.117 & 0.0005 & 0.0022 & 0.062 & 0.268 & 0.089 & 0.385 \\
17 & 23.1 & 0.100 & 0.433 & 0.320 & 1.385 & 0.0014 & 0.0061 & 0.070 & 0.303 & 0.086 & 0.372 \\
18 & 25.1 & 0.116 & 0.462 & 0.324 & 1.291 & 0.0026 & 0.0104 & 0.052 & 0.207 & 0.096 & 0.382 \\
19 & 25.2 & 0.126 & 0.500 & 0.309 & 1.226 & 0.0027 & 0.0107 & 0.096 & 0.381 & 0.106 & 0.421 \\
20 & 24.9 & 0.106 & 0.426 & 0.347 & 1.394 & 0.0031 & 0.0124 & 0.098 & 0.394 & 0.100 & 0.402 \\
\hline Mean & 24.6 & 0.109 & 0.441 & 0.307 & 1.249 & 0.0020 & 0.0082 & 0.075 & 0.304 & 0.094 & 0.382 \\
SEM & 0.34 & 0.003 & 0.012 & 0.0140 & 0.053 & 0.0003 & 0.0011 & 0.0048 & 0.019 & 0.0062 & 0.023 \\
\hline
\end{tabular}


Figure 5 (S.A.B.). Mean \pm S.E.M heart weight and heart weight as a percentage of final body weight (See also Tables 7and 8).
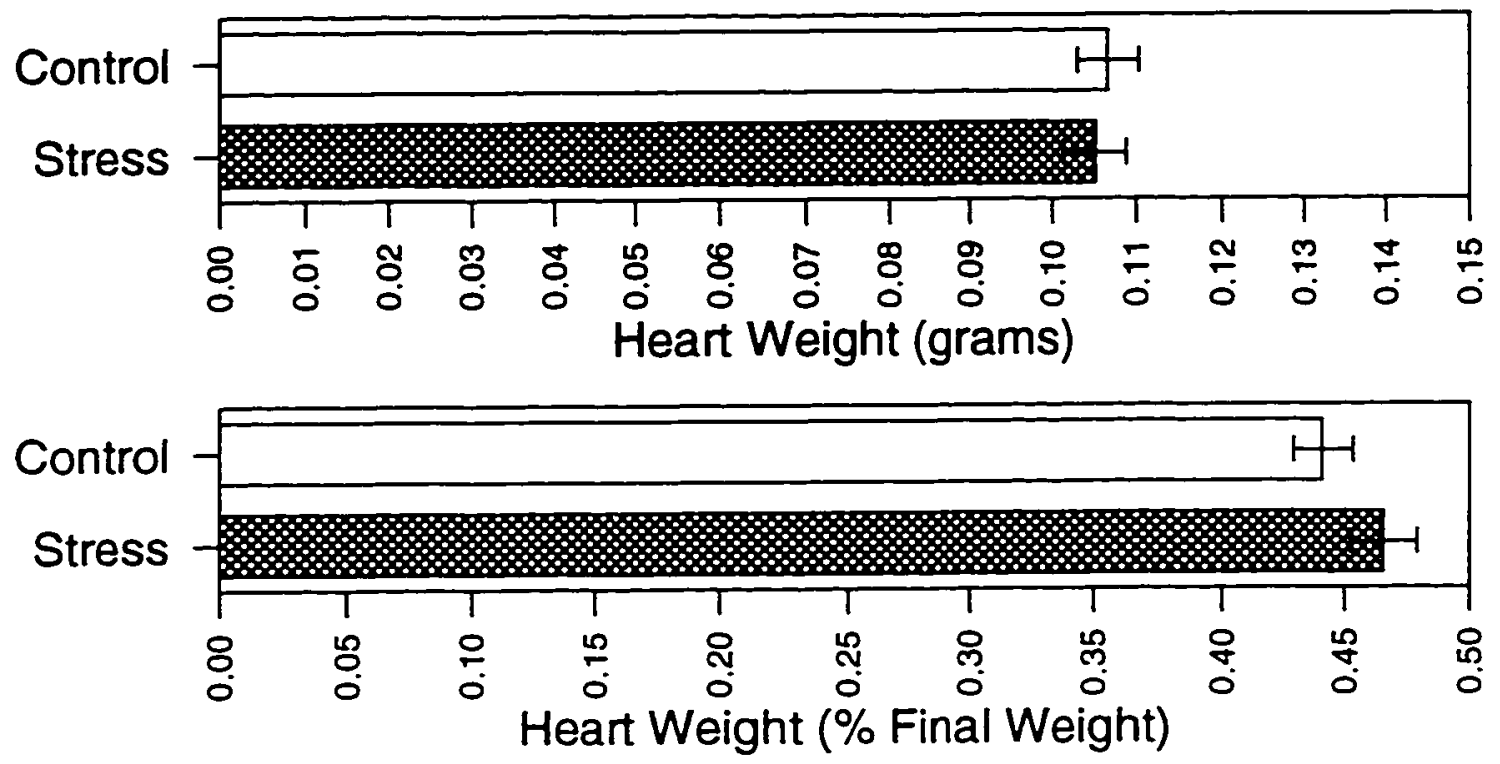

Figure 6 (S.A.B.). Mean \pm S.E.M. kidney weight and kidney weight as a percentage of final body weight (See also Tables 7 and 8 ).
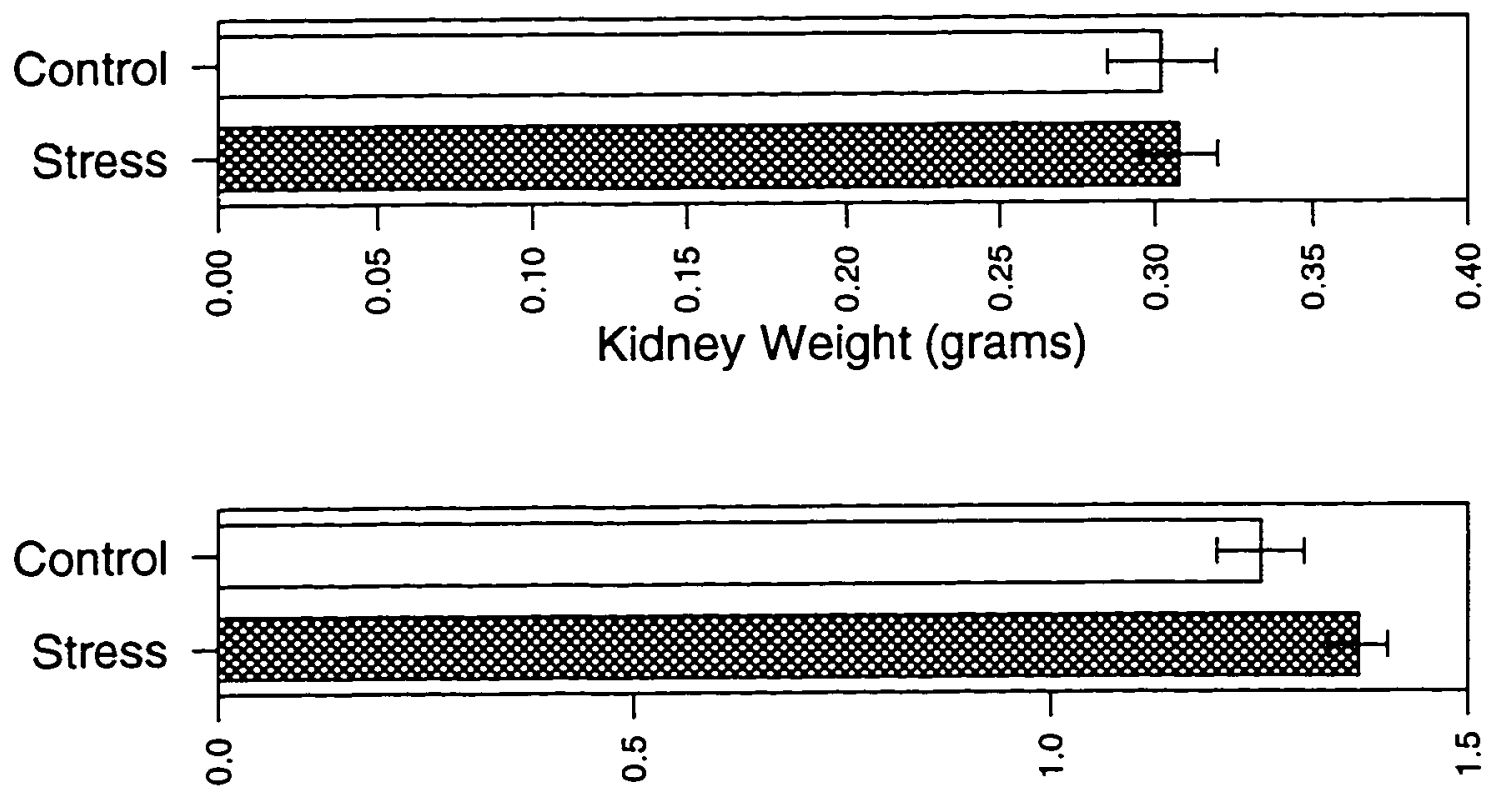

Kidney Weight (\% Final Weight) 
Figure 7 (S.A.B.). Mean \pm S.E.M. adrenal weight and adrenal weight as a percentage of final body weight (See also Tables 7and 8).
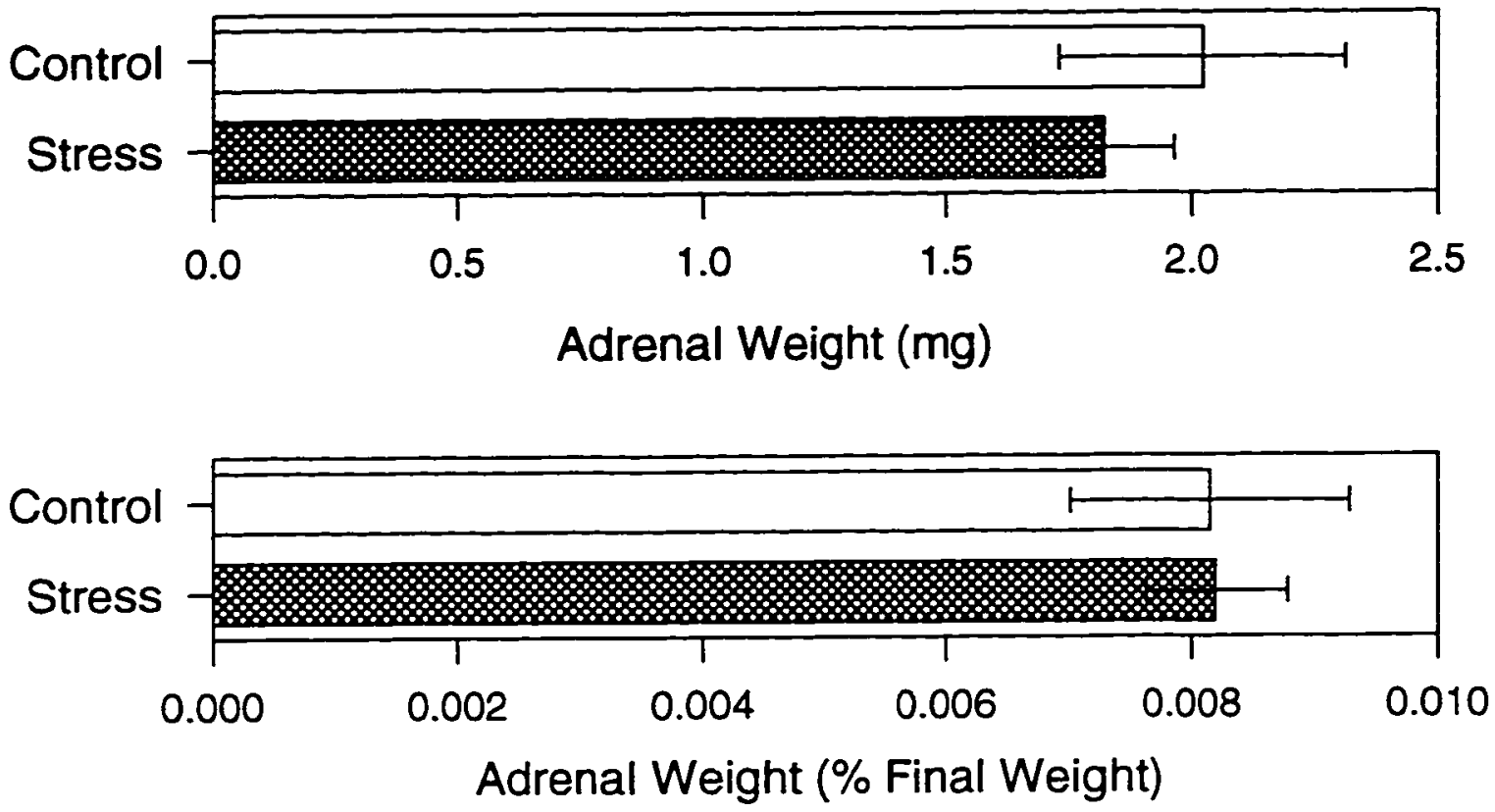

Figure 8 (S.A.B.). Mean \pm S.E.M. spleen weight and spleen weight as a percentage of final body weight (See also Tables 7 and 8 ).
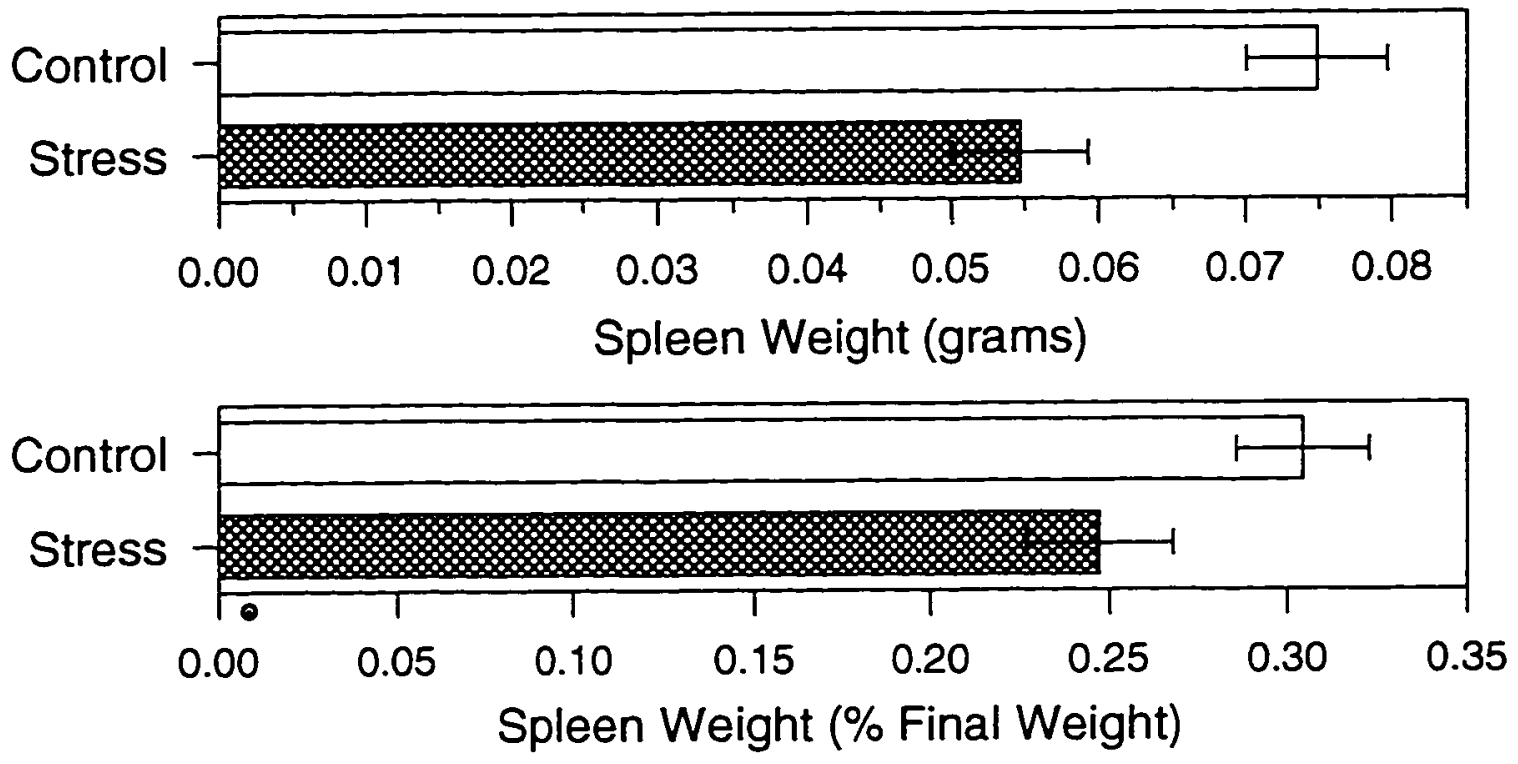
Figure 9 (S.A.B.). Mean \pm S.E.M. thymus weight and thymus weight as a percentage of final body weight (See also Tables 7 and 8 ).
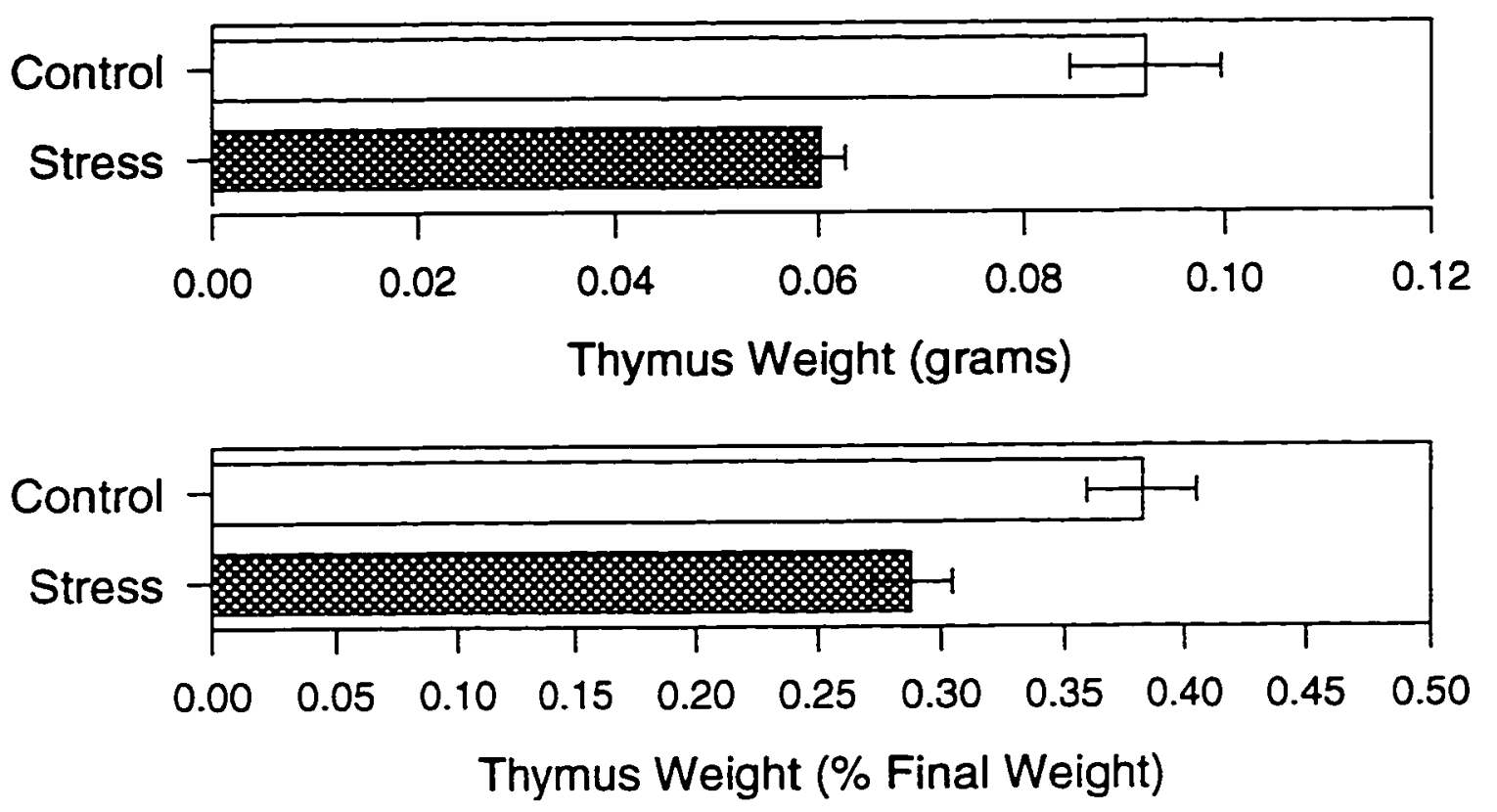
Table 9 (S.A.B.). Plasma corticosterone ( $\mu \mathrm{g} / \mathrm{dl})$, plasma protein, and total leukocyte count in the stressed and control groups.

\begin{tabular}{rlll}
$\begin{array}{c}\text { Mouse } \\
\#\end{array}$ & $\begin{array}{l}\text { Plasma } \\
\text { Corticosterone } \\
\mu \mathrm{g} / \mathrm{dl}\end{array}$ & $\begin{array}{l}\text { Plasma } \\
\text { Protein } \\
\text { mg/dl }\end{array}$ & $\begin{array}{l}\text { Total } \\
\text { WBC } \\
\text { cells/ml }\end{array}$ \\
\hline Stress 1 & 2.08 & 5.13 & 7500 \\
Group 2 & 6.10 & 6.36 & 6750 \\
3 & 9.05 & 4.10 & 2000 \\
4 & 3.86 & 3.67 & 8750 \\
5 & 3.18 & 6.36 & 7500 \\
6 & 13.71 & 6.41 & 2750 \\
7 & 7.20 & 5.86 & 5500 \\
8 & 41.52 & 4.24 & 4750 \\
9 & 9.77 & 5.55 & 8750 \\
10 & 4.50 & 4.41 & 5750 \\
\hline Mean & 10.10 & 5.21 & 6000 \\
SEM & 3.66 & 0.33 & 735.0 \\
\hline
\end{tabular}

\begin{tabular}{clll}
$\begin{array}{c}\text { Mouse } \\
\#\end{array}$ & $\begin{array}{l}\text { Plasma } \\
\text { Corticosterone } \\
\mu \mathrm{g} / \mathrm{dl}\end{array}$ & $\begin{array}{l}\text { Plasma } \\
\text { Protein } \\
\mathrm{mg} / \mathrm{dl}\end{array}$ & $\begin{array}{l}\text { Total } \\
\text { WBC } \\
\text { cells/ml }\end{array}$ \\
\hline Control11 & 1.68 & 3.23 & 2500 \\
Group 12 & 2.42 & 5.22 & 7000 \\
13 & 6.70 & 5.17 & 7250 \\
14 & 1.16 & 4.07 & 3500 \\
15 & 3.07 & 4.15 & $\mathrm{ND}$ \\
16 & 5.62 & 3.74 & 6250 \\
17 & 22.20 & 3.46 & 9250 \\
18 & 12.36 & 4.07 & 5750 \\
19 & 19.11 & 5.10 & 6250 \\
20 & 9.19 & 3.33 & 5250 \\
\hline Mean & 8.35 & 4.15 & 5889 \\
SEM & 2.34 & 0.24 & 669.4 \\
\hline
\end{tabular}

ND $=$ not determined 
Figure 10 (S.A.B.). Plasma corticosterone mean \pm S.E.M. in the stressed and control groups (See also Table 9).

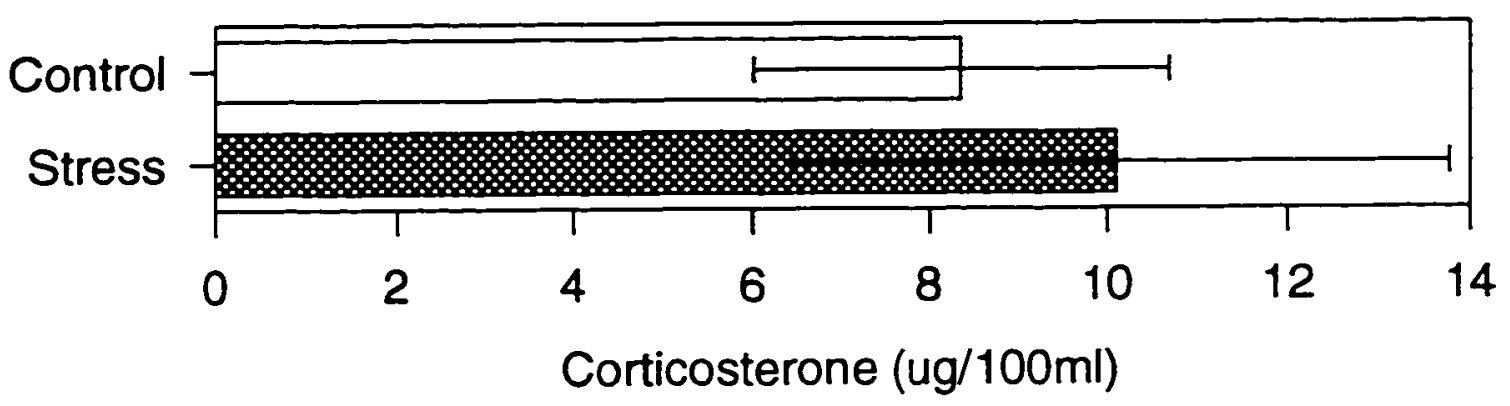

Figure 11 (S.A.B.). Plasma protein mean \pm S.E.M. (See also Table 9).

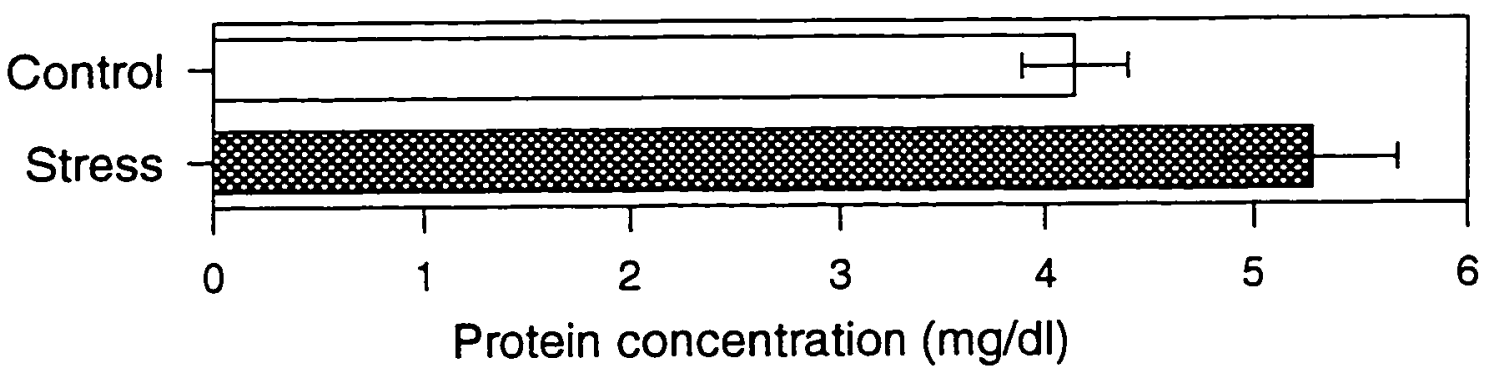


Table 10 (S.A.B.). Differential WBC count, total eosinophil count and neutrophil to lymphocyte ratio of the stress group.

\begin{tabular}{|c|c|c|c|c|c|c|c|}
\hline $\begin{array}{l}\text { Mouse } \\
\#\end{array}$ & $\begin{array}{c}\text { Eosinophil } \\
\% \text { WC }\end{array}$ & $\begin{array}{l}\text { Basophil } \\
\% W B C\end{array}$ & $\begin{array}{l}\text { Neutrophil } \\
\% \text { WBC }\end{array}$ & $\begin{array}{c}\text { Monocyte } \\
\% W B C\end{array}$ & $\begin{array}{c}\text { Lymphocyte } \\
\% W B C\end{array}$ & $\begin{array}{c}\text { Eosinophil } \\
\text { cells/ } \mu \mid\end{array}$ & N/L \\
\hline$\overline{1}$ & 0 & 0 & 5 & 3 & 92 & 0 & 0.05 \\
\hline 2 & 3 & 0 & 6 & 2 & 89 & 203 & 0.07 \\
\hline 3 & 0 & 0 & 8 & 5 & 87 & 0 & 0.09 \\
\hline 4 & 1 & 0 & 2 & 9 & 88 & 88 & 0.02 \\
\hline 5 & 2 & 0 & 8 & 5 & 85 & 150 & 0.09 \\
\hline 6 & 2 & 0 & 15 & 7 & 76 & 55 & 0.20 \\
\hline 7 & 0 & 0 & 14 & 9 & 77 & 0 & 0.18 \\
\hline 8 & 0 & 0 & 6 & 4 & 90 & 0 & 0.07 \\
\hline 9 & 1 & 0 & 12 & 5 & 82 & 88 & 0.15 \\
\hline 10 & 4 & 0 & 7 & 6 & 83 & 230 & 0.08 \\
\hline $\begin{array}{l}\text { Mean } \\
\text { SEM }\end{array}$ & $\begin{array}{l}1.3 \\
0.448\end{array}$ & & $\begin{array}{l}8.3 \\
1.309\end{array}$ & $\begin{array}{l}5.5 \\
0.734\end{array}$ & $\begin{array}{l}84.9 \\
1.703\end{array}$ & $\begin{array}{l}81 \\
27.7\end{array}$ & $\begin{array}{l}0.10 \\
0.018\end{array}$ \\
\hline
\end{tabular}

Table 11 (S.A.B.). Differential WBC count, total eosinophil count and neutrophil to lymphocyte ratio of the control group.

\begin{tabular}{|c|c|c|c|c|c|c|c|}
\hline $\begin{array}{l}\text { Mouse } \\
\#\end{array}$ & $\begin{array}{l}\text { Eosinophil } \\
\% W B C\end{array}$ & $\begin{array}{l}\text { Basophil } \\
\% W B C\end{array}$ & $\begin{array}{c}\text { Neutrophil } \\
\% W B C\end{array}$ & $\begin{array}{c}\text { Monocyte } \\
\% W B C\end{array}$ & $\begin{array}{l}\text { Lymphocyte } \\
\% W B C\end{array}$ & $\begin{array}{c}\text { Eosinophil } \\
\text { cells/ } \mu \mathrm{l}\end{array}$ & $N / L$ \\
\hline$\overline{11}$ & 4 & 0 & 13 & 4 & 79 & 100 & 0.16 \\
\hline 12 & ND & ND & ND & ND & ND & ND & ND \\
\hline 13 & 4 & 0 & 1 & 4 & 91 & 290 & 0.01 \\
\hline 14 & 0 & 0 & 8 & 2 & 90 & 0 & 0.09 \\
\hline 15 & ND & ND & ND & ND & ND & ND & ND \\
\hline 16 & 1 & 0 & 11 & 4 & 84 & 63 & 0.13 \\
\hline 17 & 3 & 0 & 10 & 6 & 81 & 288 & 0.12 \\
\hline 18 & 1 & 0 & 8 & 7 & 84 & 58 & 0.01 \\
\hline 19 & 4 & 0 & 2 & 7 & 87 & 250 & 0.02 \\
\hline$\underline{20}$ & 0 & 0 & 3 & 5 & 92 & 0 & 0.03 \\
\hline $\begin{array}{l}\text { Mean } \\
\text { SEM }\end{array}$ & $\begin{array}{l}2.1 \\
0.572\end{array}$ & & $\begin{array}{l}7 \\
1.414\end{array}$ & $\begin{array}{l}4.9 \\
0.546\end{array}$ & $\begin{array}{l}86 \\
1.512\end{array}$ & $\begin{array}{c}131 \\
44.2\end{array}$ & $\begin{array}{l}0.07 \\
0.022\end{array}$ \\
\hline
\end{tabular}

$N D=$ not determined 


\section{B. Experiment 1: Mouse Density Study}

Table 12 (Experiment 1). Daily total and per mouse water consumption for each cage (ml). Mean \pm S.E.M. were calculated using days $4-30$ only.

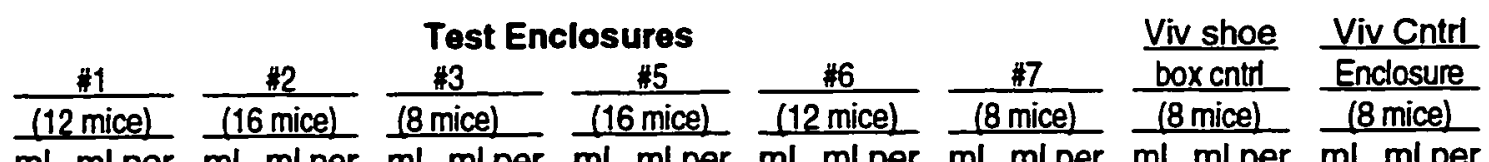

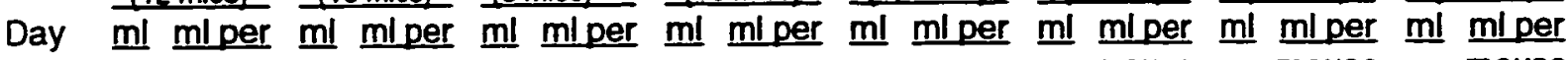

\begin{tabular}{|c|c|c|c|c|c|c|c|c|c|c|c|c|c|c|c|c|}
\hline & & mou & & ou & & $\mathrm{mou}$ & & mouse & & mouse & & mouse & & mouse & & mouse \\
\hline 1 & 16 & 1.3 & 22 & 1.4 & 15 & 1.9 & 16 & 1.0 & 20 & 1.7 & 14 & 1.8 & 41 & 5.1 & 38 & 4.8 \\
\hline 2 & 44 & 3.7 & 58 & 3.6 & 35 & 4.4 & 76 & 4.8 & 40 & 3.3 & 28 & 3.5 & & & 97 & 12.1 \\
\hline 3 & 44 & 3.7 & 62 & 3.9 & 70 & 8.8 & 71 & 4.4 & 52 & 4.3 & 29 & 3.6 & 31 & 3.9 & 30 & 3.8 \\
\hline 4 & 54 & 4.5 & 82 & 5.1 & 47 & 5.9 & 95 & 5.9 & 68 & 5.7 & 35 & 4.4 & 65 & 8.1 & 54 & 6.8 \\
\hline 5 & 56 & 4.7 & 83 & 5.2 & 50 & 6.3 & 103 & 6.4 & 67 & 5.6 & 43 & 5.4 & & & 70 & 8.8 \\
\hline 6 & 67 & 5.6 & 103 & 6.4 & 71 & 8.9 & 118 & 7.4 & 79 & 6.6 & 44 & 5.5 & 66 & 8.3 & 30 & 3.8 \\
\hline 7 & 60 & 5.0 & 96 & 6.0 & 56 & 7.0 & 102 & 6.4 & 76 & 6.3 & 40 & 5.0 & 41 & 5.1 & 30 & 3.8 \\
\hline 8 & 76 & 6.3 & 98 & 6.1 & 78 & 9.8 & 120 & 7.5 & 84 & 7.0 & 48 & 6.0 & 85 & 10.6 & 58 & 7.3 \\
\hline 9 & 75 & 6.3 & 91 & 5.7 & 64 & 8.0 & 107 & 6.7 & 82 & 6.8 & 48 & 6.0 & 84 & 10.5 & 74 & 9.3 \\
\hline 10 & 73 & 6.1 & 121 & 7.6 & 74 & 9.3 & 118 & 7.4 & 98 & 8.2 & 54 & 6.8 & 44 & 5.5 & 46 & 5.8 \\
\hline 11 & 59 & 4.9 & 112 & 7.0 & 74 & 9.3 & 116 & 7.3 & 74 & 6.2 & 60 & 7.5 & 75 & 9.4 & 56 & 7.0 \\
\hline 12 & 61 & 5.1 & 110 & 6.9 & 71 & 8.9 & 105 & 6.6 & 83 & 6.9 & 50 & 6.3 & 68 & 8.5 & 54 & 6.8 \\
\hline 13 & 84 & 7.0 & 128 & 8.0 & 88 & 11.0 & 148 & 9.3 & 94 & 7.8 & 57 & 7.1 & 70 & 8.8 & 64 & 8.0 \\
\hline 14 & 62 & 5.2 & 100 & 6.3 & 64 & 8.0 & 84 & 5.3 & 63 & 5.3 & 40 & 5.0 & 62 & 7.8 & 40 & 5.0 \\
\hline 15 & 76 & 6.3 & 120 & 7.5 & 81 & 10.1 & 122 & 7.6 & 77 & 6.4 & 104 & 13.0 & 68 & 8.5 & 62 & 7.8 \\
\hline 16 & 58 & 4.8 & 91 & 5.7 & 78 & 9.8 & 94 & 5.9 & 63 & 5.3 & 52 & 6.5 & 73 & 9.1 & 48 & 6.0 \\
\hline 17 & 78 & 6.5 & 97 & 6.1 & 28 & 3.5 & 92 & 5.8 & 73 & 6.1 & 101 & 12.6 & 49 & 6.1 & 54 & 6.8 \\
\hline 18 & 76 & 6.3 & 155 & 9.7 & 63 & 7.9 & 100 & 6.3 & 75 & 6.3 & 42 & 5.3 & 20 & 2.5 & 56 & 7.0 \\
\hline 19 & 74 & 6.2 & 53 & 3.3 & 71 & 8.9 & 84 & 5.3 & 76 & 6.3 & 48 & 6.0 & 63 & 7.9 & 64 & 8.0 \\
\hline 20 & 78 & 6.5 & 107 & 6.7 & 70 & 8.8 & 108 & 6.8 & 92 & 7.7 & 58 & 7.3 & 69 & 8.6 & 64 & 8.0 \\
\hline 21 & 66 & 5.5 & 96 & 6.0 & 45 & 5.6 & 88 & 5.5 & 79 & 6.6 & 39 & 4.9 & 64 & 8.0 & 58 & 7.3 \\
\hline 22 & 86 & 7.2 & 118 & 7.4 & 72 & 9.0 & 112 & 7.0 & 90 & 7.5 & 49 & 6.1 & 66 & 8.3 & 63 & 7.9 \\
\hline 23 & 74 & 6.2 & 107 & 6.7 & 80 & 10.0 & 98 & 6.1 & 74 & 6.2 & 51 & 6.4 & 74 & 9.3 & 57 & 7.1 \\
\hline 24 & 94 & 7.8 & 126 & 7.9 & 84 & 10.5 & 130 & 8.1 & 93 & 7.8 & 62 & 7.8 & 70 & 8.8 & 86 & 10.8 \\
\hline 25 & 82 & 6.8 & 110 & 6.9 & 58 & 7.3 & 108 & 6.8 & 81 & 6.8 & 53 & 6.6 & 68 & 8.5 & 69 & 8.6 \\
\hline 26 & 85 & 7.1 & 117 & 7.3 & 74 & 9.3 & 117 & 7.3 & 80 & 6.7 & 60 & 7.5 & 64 & 8.0 & 60 & 7.5 \\
\hline 27 & 77 & 6.4 & 118 & 7.4 & 69 & 8.6 & 121 & 7.6 & 78 & 6.5 & 55 & 6.9 & 48 & 6.0 & 62 & 7.8 \\
\hline 28 & 88 & 7.3 & 116 & 7.3 & 78 & 9.8 & 125 & 7.8 & 83 & 6.9 & 60 & 7.5 & 52 & 6.5 & 57 & 7.1 \\
\hline 29 & 80 & 6.7 & 136 & 8.5 & 75 & 9.4 & 128 & 8.0 & 93 & 7.8 & 58 & 7.3 & 20 & 2.5 & 54 & 6.8 \\
\hline 30 & 82 & 6.8 & 106 & 6.6 & 74 & 9.3 & 112 & 7.0 & 91 & 7.6 & 46 & 5.8 & 45 & 5.6 & 68 & 8.5 \\
\hline & & 6.1 & 107 & 6.7 & 68 & 8.5 & 109 & 6.8 & 80 & 6.7 & 54 & 6.7 & 60 & 7.6 & 58 & 7.2 \\
\hline & 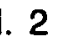 & 0.2 & 7 & 0.2 & 3 & 0.3 & 3 & 0.2 & 2 & 0.2 & 3 & 0.4 & 3 & 0.4 & 2 & 0.3 \\
\hline
\end{tabular}


Figure 12 (Experiment 1). Mean \pm S.E.M. daily water consumption per cage and per mouse (See Table 22).
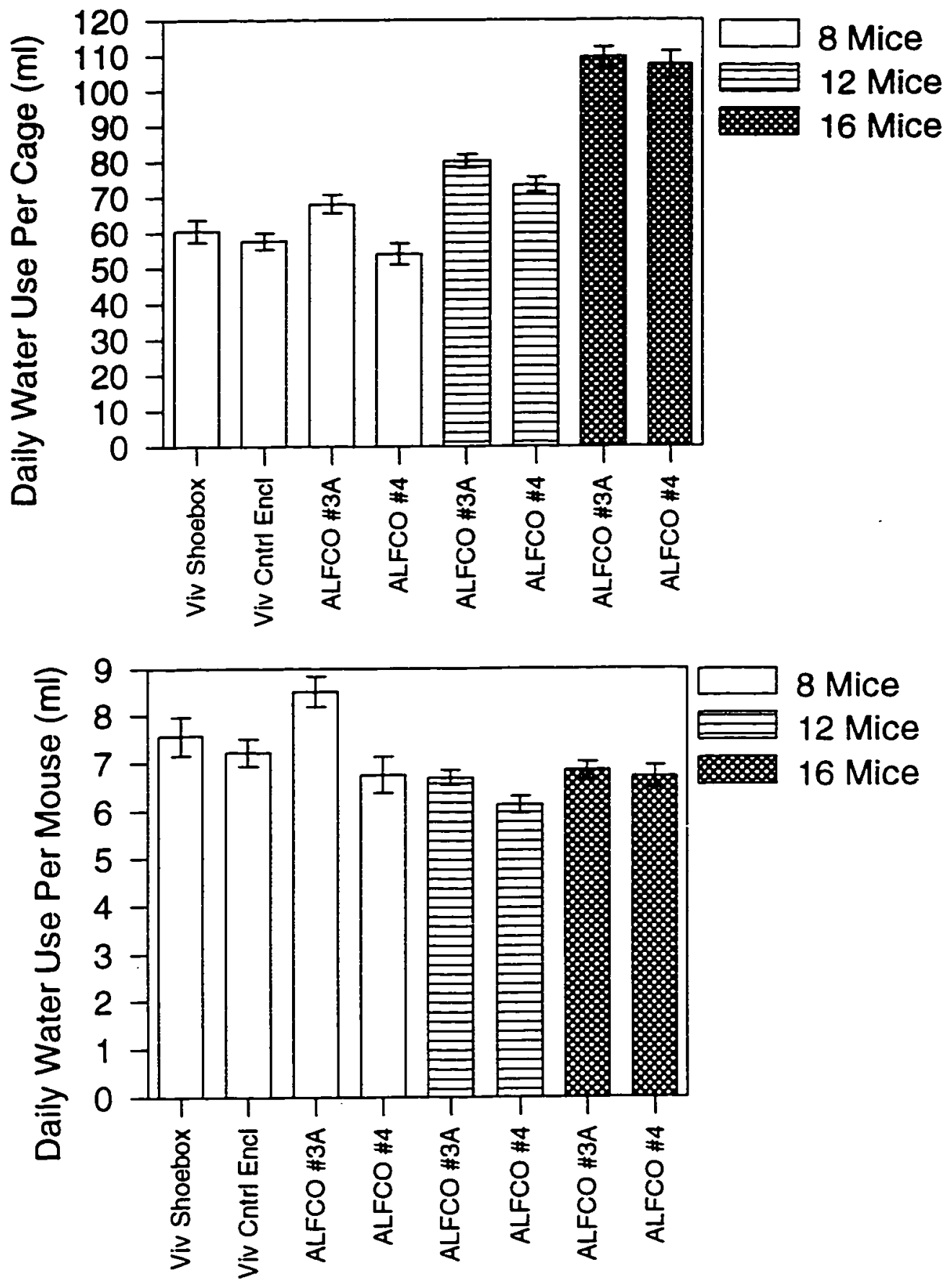
Table 13 (Experiment 1). Total food use (grams) over 30 days.

\begin{tabular}{lrrrrrr} 
& $\begin{array}{c}\text { Test } \\
\text { Encl \#3 }\end{array}$ & $\begin{array}{c}\text { Test } \\
\text { Encl \#6 } \\
(8 \text { mice })\end{array}$ & $\begin{array}{c}\text { Test } \\
\text { Encl \#5 }\end{array}$ & $\begin{array}{c}\text { Test } \\
\text { Encl \#7 } \\
(12 \text { mice })\end{array}$ & $\begin{array}{c}\text { Test } \\
\text { Encl \#1 }\end{array}$ & $\begin{array}{c}\text { Test } \\
\text { Encl \#2 }\end{array}$ \\
& $(8$ mice $)$ & $(12$ mice $)$ & \multicolumn{1}{c}{$(16$ mice $)$} \\
\hline Total & 690.83 & 665.17 & 666.11 & 724.75 & 1775.28 & 1218.38 \\
Totalmouse/day & 3.84 & 3.70 & 3.70 & 4.03 & 4.93 & 3.38
\end{tabular}

Table 14 (Experiment 1). Mouse initial (day 0 ) and final (day 30) body weight, weight gain in grams, and \% weight gain.

\begin{tabular}{lccccc} 
& $\begin{array}{c}\text { Mouse } \\
\#\end{array}$ & $\begin{array}{c}\text { Initial } \\
\text { Wt }(\mathrm{g})\end{array}$ & $\begin{array}{c}\text { Final } \\
\text { Wt }(\mathrm{g})\end{array}$ & $\begin{array}{c}\text { Gain } \\
(\mathrm{g})\end{array}$ & $\begin{array}{c}\text { Gain } \\
\text { (\% Initial Weight) }\end{array}$ \\
\hline Test enclosure \#3 & 1 & 21.59 & 35.09 & 13.50 & 62.53 \\
ALFCO \#3A Filter & 2 & 23.71 & 30.66 & 6.95 & 29.31 \\
8 mice & 3 & 21.61 & 28.17 & 6.56 & 30.36 \\
& 4 & 22.88 & 32.60 & 9.72 & 42.48 \\
& 5 & 21.60 & 30.32 & 8.72 & 40.37 \\
& 6 & 23.56 & 29.13 & 5.57 & 23.64 \\
& 7 & 21.54 & 27.53 & 5.99 & 27.81 \\
Mean & 8 & 21.43 & 25.76 & 4.33 & 20.21 \\
SEM & & 22.24 & 29.91 & 7.67 & 34.59 \\
& & 0.35 & 1.05 & 1.03 & 4.81 \\
\hline Test enclosure \#6 & & & & & \\
ALFCO \#3A Filter & Initial & Final & Gain & Gain \\
12 mice & 10 & 21.81 & 30.31 & 8.50 & 38.97 \\
& 11 & 21.46 & 26.24 & 4.78 & 22.27 \\
& 12 & 21.07 & 30.13 & 9.06 & 43.00 \\
& 13 & 21.69 & 26.53 & 4.84 & 22.31 \\
& 14 & 21.50 & 23.87 & 2.37 & 11.02 \\
& 15 & 23.28 & 33.08 & 9.80 & 42.10 \\
& 16 & 23.17 & 31.38 & 8.21 & 35.43 \\
& 17 & 21.04 & 31.43 & 10.39 & 49.38 \\
Mean & 18 & 20.22 & 27.70 & 7.48 & 36.99 \\
& 19 & 21.07 & 31.30 & 10.23 & 48.55 \\
& 20 & 22.24 & 29.60 & 7.36 & 33.09 \\
\hline & & 21.82 & 29.22 & 7.40 & 33.99 \\
& & 0.29 & 0.77 & 0.72 & 3.39 \\
\hline & & & & &
\end{tabular}




\begin{tabular}{lccccc} 
& $\begin{array}{c}\text { Mouse } \\
\#\end{array}$ & $\begin{array}{c}\text { Initial } \\
\text { Wt }(\mathrm{g})\end{array}$ & $\begin{array}{c}\text { Final } \\
\text { Wt }(\mathrm{g})\end{array}$ & $\begin{array}{c}\text { Gain } \\
(\mathrm{g})\end{array}$ & $\begin{array}{c}\text { Gain } \\
\text { (\% Initial Weight) }\end{array}$ \\
\hline Test enclosure $\$ 5$ & 21 & 21.21 & 25.42 & 4.21 & 19.85 \\
ALFCO \#3A Filter & 22 & 20.78 & 27.23 & 6.45 & 31.04 \\
16 mice & 23 & 21.79 & 32.28 & 10.49 & 48.14 \\
& 24 & 23.54 & 34.23 & 10.69 & 45.41 \\
& 25 & 23.66 & 31.37 & 7.71 & 32.59 \\
& 26 & 22.22 & 25.77 & 3.55 & 15.98 \\
& 27 & 22.91 & 30.50 & 7.59 & 33.13 \\
& 28 & 22.50 & 26.53 & 4.03 & 17.91 \\
& 29 & 22.12 & 28.00 & 5.88 & 26.58 \\
& 30 & 20.72 & 34.09 & 13.37 & 64.53 \\
& 31 & 21.15 & 24.84 & 3.69 & 17.45 \\
& 32 & 20.08 & 25.66 & 5.58 & 27.79 \\
& 33 & 21.35 & 24.52 & 3.17 & 14.85 \\
& 34 & 21.25 & 26.38 & 5.13 & 24.14 \\
& 35 & 21.14 & 29.67 & 8.53 & 40.35 \\
Mean & 36 & 24.01 & 29.84 & 5.83 & 24.28 \\
\hline SEM & & 21.90 & 28.52 & 6.62 & 30.25 \\
\hline
\end{tabular}

\begin{tabular}{lccccc} 
& $\begin{array}{c}\text { Mouse } \\
\#\end{array}$ & $\begin{array}{c}\text { Initial } \\
\text { Wt }(\mathrm{g})\end{array}$ & $\begin{array}{c}\text { Final } \\
\text { Wt }(\mathrm{g})\end{array}$ & $\begin{array}{c}\text { Gain } \\
(\mathrm{g})\end{array}$ & $\begin{array}{c}\text { Gain } \\
\text { (\% Initial Weight) }\end{array}$ \\
\hline Test enclosure \#7 & 37 & 19.80 & 25.49 & 5.69 & 28.74 \\
ALFCO \#4 Filter & 38 & 22.66 & 30.10 & 7.44 & 32.83 \\
8 mice & 39 & 20.98 & 29.04 & 8.06 & 38.42 \\
& 40 & 21.21 & 27.59 & 6.38 & 30.08 \\
& 41 & 20.77 & 26.22 & 5.45 & 26.24 \\
& 42 & 22.22 & 31.63 & 9.41 & 42.35 \\
& 43 & 23.56 & 30.45 & 6.89 & 29.24 \\
\hline Mean & 44 & 20.47 & 26.99 & 6.52 & 31.85 \\
SEM & & 21.46 & 28.44 & 6.98 & 32.47 \\
\hline
\end{tabular}




\begin{tabular}{lccccc} 
& $\begin{array}{c}\text { Mouse } \\
\#\end{array}$ & $\begin{array}{c}\text { Initial } \\
\text { Wt }(\mathrm{g})\end{array}$ & $\begin{array}{c}\text { Final } \\
\text { Wt }(\mathrm{g})\end{array}$ & $\begin{array}{c}\text { Gain } \\
(\mathrm{g})\end{array}$ & $\begin{array}{c}\text { Gain } \\
\text { (\% Initial Weight) }\end{array}$ \\
\hline Test enclosure \#1 & 45 & 20.19 & 27.19 & 7.00 & 34.67 \\
ALFCO \#4 Fitter & 46 & 21.24 & 30.80 & 9.56 & 45.01 \\
12 mice & 47 & 21.06 & 27.24 & 6.18 & 29.34 \\
& 48 & 22.00 & 27.62 & 5.62 & 25.55 \\
& 49 & 22.46 & 24.74 & 2.28 & 10.15 \\
& 50 & 22.55 & 28.77 & 6.22 & 27.58 \\
& 51 & 22.42 & 27.22 & 4.80 & 21.41 \\
& 52 & 20.54 & 31.24 & 10.70 & 52.09 \\
& 53 & 22.54 & 31.31 & 8.77 & 38.91 \\
& 54 & 21.01 & 26.75 & 5.74 & 27.32 \\
& 55 & 23.91 & 30.55 & 6.64 & 27.77 \\
Mean & 56 & 21.82 & 28.03 & 6.21 & 28.46 \\
\hline
\end{tabular}

\begin{tabular}{lccccc} 
& $\begin{array}{c}\text { Mouse } \\
\#\end{array}$ & $\begin{array}{c}\text { Initial } \\
\text { Wt }(g)\end{array}$ & $\begin{array}{c}\text { Final } \\
\text { Wt (g) }\end{array}$ & $\begin{array}{c}\text { Gain } \\
(\mathrm{g})\end{array}$ & $\begin{array}{c}\text { Gain } \\
\text { (\% Initial Weight) }\end{array}$ \\
\hline Test enclosure *2 & 57 & 21.09 & 27.08 & 5.99 & 28.40 \\
ALFCO \#4 Filter & 58 & 21.95 & 27.27 & 5.32 & 24.24 \\
16 mice & 59 & 20.55 & 26.79 & 6.24 & 30.36 \\
& 60 & 21.42 & 29.29 & 7.87 & 36.74 \\
& 61 & 20.83 & 29.36 & 8.53 & 40.95 \\
& 62 & 21.44 & 27.26 & 5.82 & 27.15 \\
& 63 & 21.56 & 27.65 & 6.09 & 28.25 \\
& 64 & 23.28 & 28.66 & 5.38 & 23.11 \\
& 65 & 20.14 & 32.89 & 12.75 & 63.31 \\
& 66 & 22.75 & 28.47 & 5.72 & 25.14 \\
& 67 & 20.38 & 26.33 & 5.95 & 29.20 \\
& 68 & 22.01 & 26.22 & 4.21 & 19.13 \\
& 69 & 21.60 & 28.11 & 6.51 & 30.14 \\
& 70 & 20.63 & 26.42 & 5.79 & 28.07 \\
& 71 & 20.46 & 26.93 & 6.47 & 31.62 \\
Mean & 72 & 22.10 & 31.26 & 9.16 & 41.45 \\
\hline
\end{tabular}




\begin{tabular}{lccccc} 
& $\begin{array}{c}\text { Mouse } \\
\#\end{array}$ & $\begin{array}{c}\text { Initial } \\
\text { Wt }(\mathrm{g})\end{array}$ & $\begin{array}{c}\text { Final } \\
\text { Wt }(\mathrm{g})\end{array}$ & $\begin{array}{c}\text { Gain } \\
(\mathrm{g})\end{array}$ & $\begin{array}{c}\text { Gain } \\
\text { (\% Initial Weight) }\end{array}$ \\
\hline Vivarium Shoe Box Control & 73 & 22.91 & 26.48 & 3.57 & 15.58 \\
8 mice & 74 & 20.34 & 24.09 & 3.75 & 18.44 \\
& 75 & 23.86 & 29.05 & 5.19 & 21.75 \\
& 76 & 22.56 & 28.34 & 5.78 & 25.62 \\
& 77 & 21.13 & 25.53 & 4.40 & 20.82 \\
& 78 & 20.83 & 28.27 & 7.44 & 35.72 \\
& 79 & 22.26 & 28.18 & 5.92 & 26.59 \\
\hline Mean & 80 & 22.50 & 29.65 & 7.15 & 31.78 \\
SEM & & 22.05 & 27.45 & 5.40 & 24.54 \\
\hline
\end{tabular}

\begin{tabular}{lccccc} 
& $\begin{array}{c}\text { Mouse } \\
\#\end{array}$ & $\begin{array}{c}\text { Initial } \\
\text { Wt }(g)\end{array}$ & $\begin{array}{c}\text { Final } \\
\text { Wt }(\mathrm{g})\end{array}$ & $\begin{array}{c}\text { Gain } \\
(\mathrm{g})\end{array}$ & $\begin{array}{c}\text { Gain } \\
\text { (\% Initial Weight) }\end{array}$ \\
\hline Vivarium Control Enclosure & 81 & 20.88 & 26.14 & 5.26 & 25.19 \\
8 mice & 82 & 21.71 & 27.98 & 6.27 & 28.88 \\
& 83 & 23.17 & 38.81 & 15.64 & 67.50 \\
& 84 & 22.51 & 29.21 & 6.70 & 29.76 \\
& 85 & 21.77 & 27.90 & 6.13 & 28.16 \\
& 86 & 22.35 & 29.62 & 7.27 & 32.53 \\
& 87 & 23.24 & 30.32 & 7.08 & 30.46 \\
\hline Mean & 88 & 20.39 & 27.70 & 7.31 & 35.85 \\
SEM & & 22.00 & 29.71 & 7.71 & 34.79 \\
\hline
\end{tabular}

Table 15 (Experiment 1). Cage mean \pm S.E.M for initial (day 0 ) and final (day 30 ) body weight, weight gain in grams, and \% weight gain.

\begin{tabular}{lcccc} 
& $\begin{array}{c}\text { Initial } \\
\text { Wt }(\mathrm{g})\end{array}$ & $\begin{array}{c}\text { Final } \\
\text { Wt }(\mathrm{g})\end{array}$ & $\begin{array}{c}\text { Gain } \\
(\mathrm{g})\end{array}$ & $\begin{array}{c}\text { Gain } \\
\text { (\% Initial Weight) }\end{array}$ \\
\hline Vivarium Shoe Box Control, 8 mice & $22.05 \pm 0.42$ & $27.45 \pm 0.67$ & $5.40 \pm 0.51$ & $24.54 \pm 2.40$ \\
Vivarium Control Enclosure, 8 mice & $22.00 \pm 0.36$ & $29.71 \pm 1.38$ & $7.71 \pm 1.16$ & $34.79 \pm 4.80$ \\
Test enclosure \#3, ALFCO \#3A Filter, 8 mice & $22.24 \pm 0.35$ & $29.91 \pm 1.05$ & $7.67 \pm 1.03$ & $34.59 \pm 4.81$ \\
Test enclosure \#7. ALFCO \#4 Filler, 8 mice & $21.46 \pm 0.44$ & $28.44 \pm 0.78$ & $6.98 \pm 0.46$ & $32.47 \pm 1.90$ \\
Test enclosure \#6. ALFCO \#3A Filter, 12 mice $21.82 \pm 0.29$ & $29.22 \pm 0.77$ & $7.40 \pm 0.72$ & $33.99 \pm 3.39$ \\
Test enclosure \#1, ALFCO \#4 Filler, 12 mice & $21.81 \pm 0.30$ & $28.46 \pm 0.60$ & $6.64 \pm 0.64$ & $30.69 \pm 3.16$ \\
Test enclosure \#5, ALFCO \#3A Filter, 16 mice $21.90 \pm 0.29$ & $28.52 \pm 0.81$ & $6.62 \pm 0.74$ & $30.25 \pm 3.42$ \\
Test enclosure \#2, ALFCO \#4 Filter, 16 mice $21.39 \pm 0.22$ & $28.12 \pm 0.46$ & $6.74 \pm 0.51$ & $31.70 \pm 2.58$
\end{tabular}


Figure 13 (Experiment 1). Mean \pm S.E.M. starting body weight (day 1) and mean \pm S.E.M. ending body weight (day 30 ). (See also table 15).
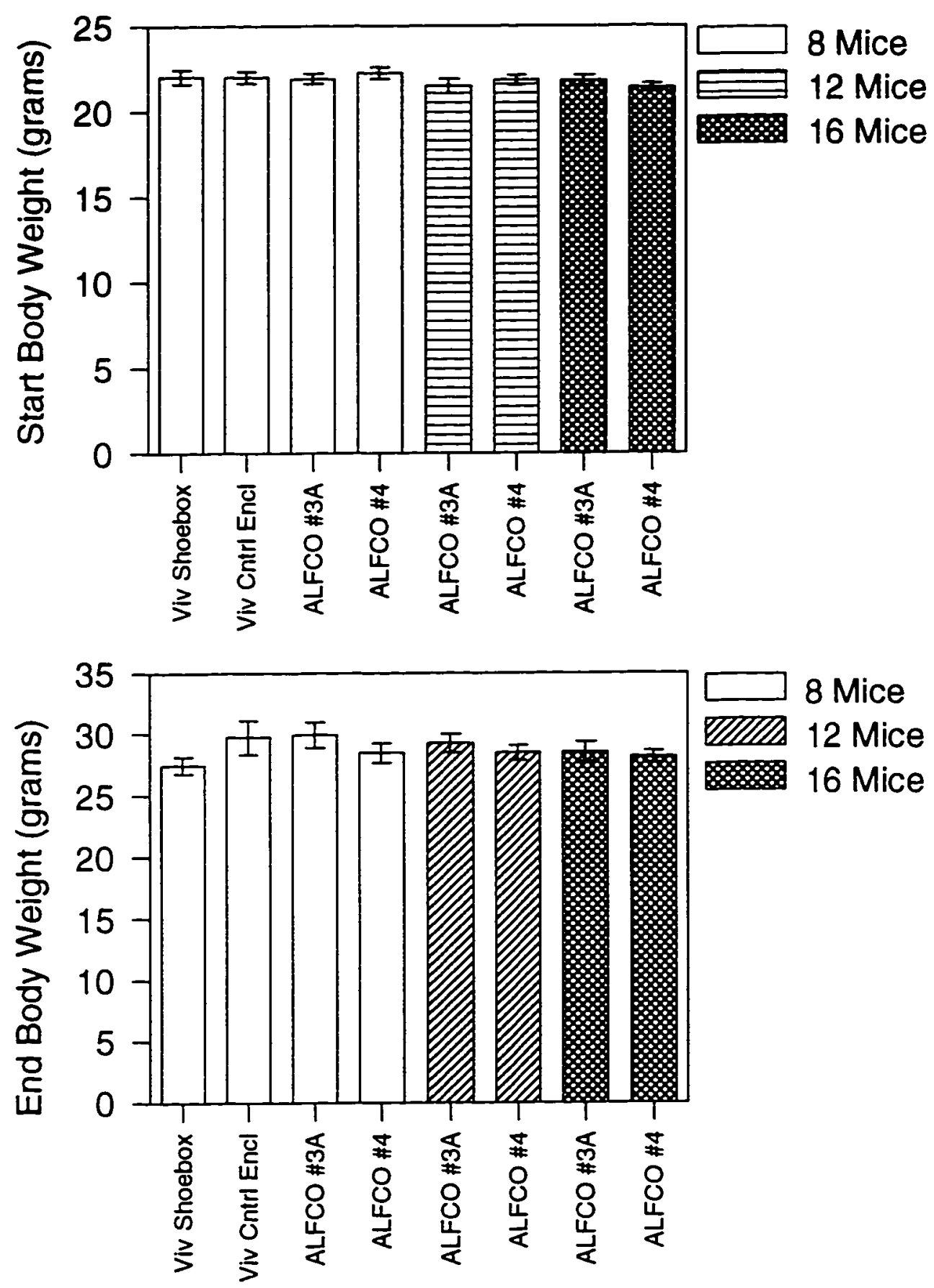
Figure 14 (Experiment 1). Mean \pm S.E.M. body weight gain and mean \pm S.E.M. per cent body weight gain. (See also table 15).
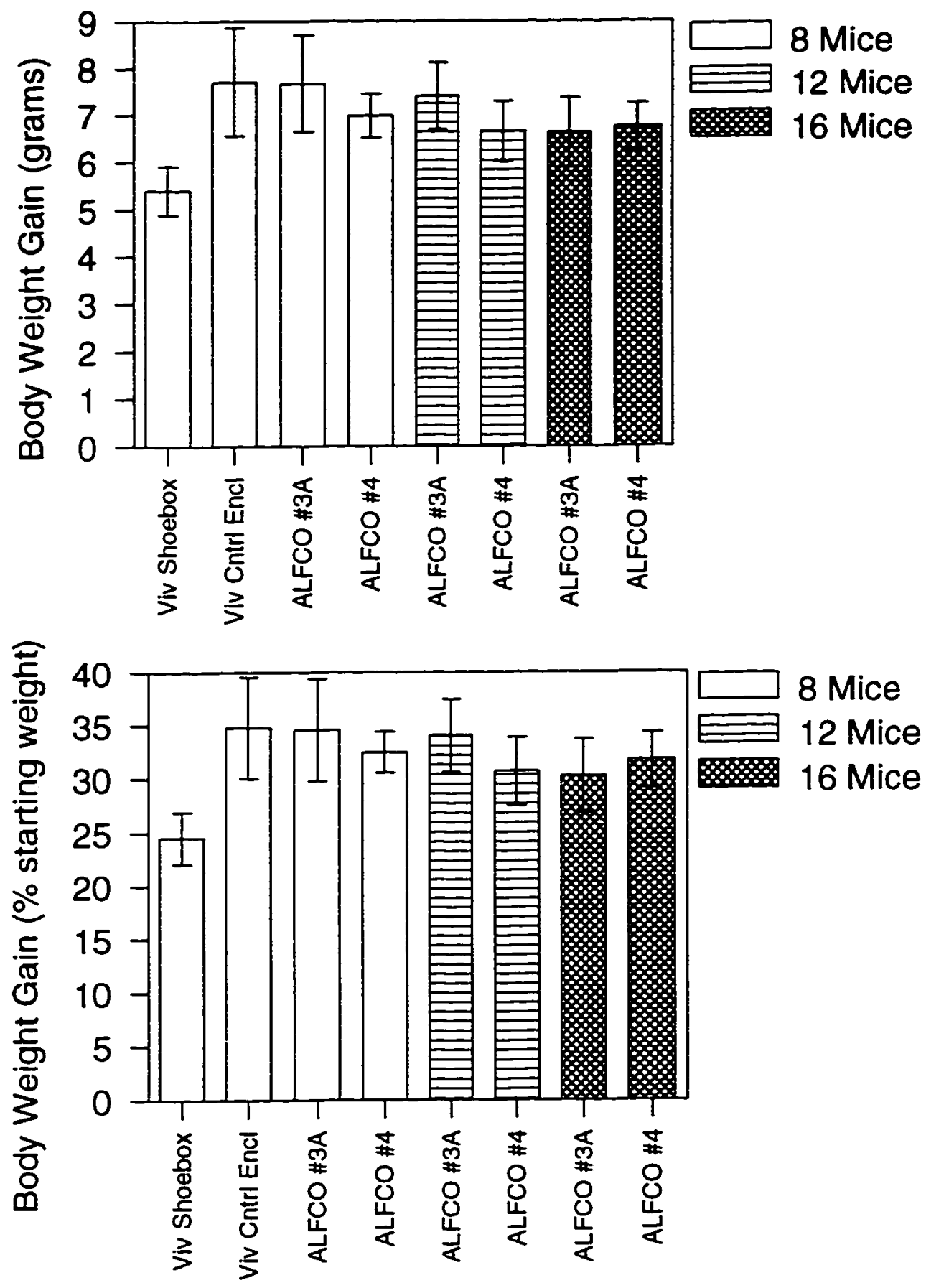
Table 16 (Experiment 1). Organ weights in grams and as a percentage of body weight (BW).

\begin{tabular}{|c|c|c|c|c|c|c|c|c|c|c|}
\hline $\begin{array}{l}\text { Test E } \\
\text { Mouse } \\
\text { id \# }\end{array}$ & $\begin{array}{l}\text { Enclosu } \\
\text { Heart } \\
\text { Wt (g) }\end{array}$ & $\begin{array}{c}\text { re } \% 3, A \\
\text { Heart } \\
\% \text { BW }\end{array}$ & $\begin{array}{l}\text { FO } \\
\text { Kidney } \\
\text { Wt (g) }\end{array}$ & $\begin{array}{l}\text { Filter, } 8 \\
\text { Kidney } \\
\% \text { BW }\end{array}$ & $\begin{array}{l}\text { nice } \\
\text { Adrenal } \\
\text { Wt (g) }\end{array}$ & $\begin{array}{c}\text { Adrenal } \\
\% \text { BW }\end{array}$ & $\begin{array}{l}\text { Spleen } \\
\text { Wt (g) }\end{array}$ & $\begin{array}{l}\text { Spleen } \\
\% \text { BW }\end{array}$ & $\begin{array}{l}\text { Thymus } \\
\text { Wt (g) }\end{array}$ & $\begin{array}{l}\text { Thymus } \\
\% \text { BW }\end{array}$ \\
\hline 1 & 0.121 & 0.345 & 0.358 & 1.020 & & & 0.062 & 0.177 & 0.052 & 0.148 \\
\hline 2 & 0.122 & 0.398 & 0.378 & 1.233 & 0.0056 & 0.0183 & 0.067 & 0.219 & 0.087 & 0.284 \\
\hline 3 & 0.116 & 0.412 & 0.384 & 1.363 & 0.0021 & 0.0075 & 0.126 & 0.447 & 0.054 & 0.192 \\
\hline 4 & 0.116 & 0.356 & 0.384 & 1.178 & 0.0028 & 0.0086 & 0.082 & 0.252 & 0.039 & 0.120 \\
\hline 5 & 0.134 & 0.442 & 0.462 & 1.524 & 0.0025 & 0.0082 & 0.112 & 0.369 & 0.125 & 0.412 \\
\hline 6 & 0.128 & 0.439 & 0.388 & 1.332 & 0.0014 & 0.0048 & 0.109 & 0.374 & 0.076 & 0.261 \\
\hline 7 & 0.120 & 0.436 & 0.417 & 1.515 & 0.0035 & 0.0127 & 0.086 & 0.312 & 0.108 & 0.392 \\
\hline 8 & 0.109 & 0.423 & 0.340 & 1.320 & 0.0038 & 0.0148 & 0.082 & 0.318 & 0.067 & 0.260 \\
\hline$\overline{\text { Mean }}$ & 0.121 & 0.406 & 0.389 & 1.311 & 0.0031 & 0.0107 & 0.091 & 0.309 & 0.076 & 0.259 \\
\hline SEM & 0.003 & 0.013 & 0.013 & 0.060 & 0.0005 & 0.0018 & 0.008 & 0.032 & 0.010 & 0.037 \\
\hline
\end{tabular}

\begin{tabular}{|c|c|c|c|c|c|c|c|c|c|c|}
\hline $\begin{array}{l}\text { Mouse } \\
\text { id \# }\end{array}$ & $\begin{array}{l}\text { Heart } \\
\text { Wt (g) }\end{array}$ & $\begin{array}{l}\text { Heart } \\
\% \text { BW }\end{array}$ & $\begin{array}{l}\text { Kidney } \\
\text { Wt (g) }\end{array}$ & $\begin{array}{l}\text { Kidney } \\
\% \text { BW }\end{array}$ & $\begin{array}{l}\text { Adrenal } \\
\text { Wt (g) }\end{array}$ & $\begin{array}{l}\text { Adrenal } \\
\% \text { BW }\end{array}$ & $\begin{array}{l}\text { Spleen } \\
\text { Wt (g) }\end{array}$ & $\begin{array}{l}\text { Spleen } \\
\% \text { BW }\end{array}$ & $\begin{array}{l}\text { Thymus } \\
\text { Wt (g) }\end{array}$ & $\begin{array}{l}\text { Thymus } \\
\% \text { BW }\end{array}$ \\
\hline 9 & 0.118 & 0.406 & 0.521 & 1.792 & 0.004 & 0.0138 & 0.121 & 0.416 & 0.090 & 0.310 \\
\hline 10 & 0.135 & 0.445 & 0.466 & 1.537 & 0.0022 & 0.0073 & 0.122 & 0.403 & 0.061 & 0.201 \\
\hline 11 & 0.108 & 0.412 & 0.323 & 1.231 & 0.002 & 0.0076 & 0.094 & 0.358 & 0.085 & 0.324 \\
\hline 12 & 0.124 & 0.412 & 0.439 & 1.457 & 0.0014 & 0.0046 & 0.110 & 0.365 & 0.087 & 0.289 \\
\hline 13 & 0.120 & 0.452 & 0.405 & 1.527 & 0.0053 & 0.0200 & 0.100 & 0.377 & 0.055 & 0.207 \\
\hline 14 & 0.125 & 0.524 & 0.386 & 1.617 & 0.0039 & 0.0163 & 0.070 & 0.293 & 0.069 & 0.289 \\
\hline 15 & 0.143 & 0.432 & 0.452 & 1.366 & 0.0023 & 0.0070 & 0.130 & 0.393 & 0.082 & 0.248 \\
\hline 16 & 0.131 & 0.417 & 0.492 & 1.568 & 0.0050 & 0.0159 & 0.109 & 0.347 & 0.100 & 0.319 \\
\hline 17 & 0.151 & 0.480 & 0.462 & 1.470 & 0.0069 & 0.0220 & 0.109 & 0.347 & 0.070 & 0.223 \\
\hline 18 & 0.131 & 0.473 & 0.302 & 1.090 & 0.0012 & 0.0043 & 0.087 & 0.314 & 0.096 & 0.347 \\
\hline 19 & 0.143 & 0.457 & 0.426 & 1.361 & 0.0047 & 0.0150 & 0.099 & 0.316 & 0.112 & 0.358 \\
\hline$\underline{20}$ & 0.146 & 0.493 & 0.412 & 1.392 & 0.0014 & 0.0047 & 0.095 & 0.321 & 0.063 & 0.213 \\
\hline $\bar{M}$ & 0.131 & 0.450 & 0.424 & 1.451 & 0.0034 & 0.0115 & 0.104 & 0.354 & 0.081 & 0.277 \\
\hline SEM & 0.004 & 0.011 & 0.019 & 0.053 & 0.0005 & 0.0018 & 0.005 & 0.011 & 0.005 & 0.016 \\
\hline
\end{tabular}




\begin{tabular}{|c|c|c|c|c|c|c|c|c|c|c|}
\hline $\begin{array}{l}\text { Mous } \\
\text { id \# }\end{array}$ & $\begin{array}{l}\text { Heart } \\
\text { Wt }(\mathrm{g})\end{array}$ & $\begin{array}{l}\text { Heart } \\
\% \text { BW }\end{array}$ & $\begin{array}{l}\text { Kidney } \\
\text { Wt (g) }\end{array}$ & $\begin{array}{l}\text { Kidney } \\
\% \text { BW }\end{array}$ & $\begin{array}{l}\text { Adrenal } \\
\text { Wt (g) }\end{array}$ & $\begin{array}{l}\text { Adrenal } \\
\% \text { BW }\end{array}$ & $\begin{array}{l}\text { Spleen } \\
\text { Wt (g) }\end{array}$ & $\begin{array}{l}\text { Spleen } \\
\% \text { BW }\end{array}$ & $\begin{array}{l}\text { Thymus } \\
\text { Wt (g) }\end{array}$ & $\begin{array}{l}\text { Thymus } \\
\% \mathrm{BW}\end{array}$ \\
\hline$\overline{21}$ & 0.116 & 0.456 & 0.362 & 1.424 & 0.0022 & 0.0087 & 0.082 & 0.323 & 0.086 & 0.338 \\
\hline 22 & 0.130 & 0.477 & 0.421 & 1.546 & 0.0023 & 0.0084 & 0.100 & 0.367 & 0.046 & 0.169 \\
\hline 23 & 0.147 & 0.455 & 0.551 & 1.707 & 0.0032 & 0.0099 & 0.089 & 0.276 & 0.060 & 0.186 \\
\hline 24 & 0.147 & 0.429 & 0.587 & 1.715 & 0.0048 & 0.0140 & 0.147 & 0.429 & 0.087 & 0.254 \\
\hline 25 & 0.126 & 0.402 & 0.393 & 1.253 & 0.0042 & 0.0134 & 0.105 & 0.335 & 0.090 & 0.287 \\
\hline 26 & 0.124 & 0.481 & 0.389 & 1.510 & 0.0034 & 0.0132 & 0.080 & 0.310 & 0.065 & 0.252 \\
\hline 27 & 0.132 & 0.433 & 0.441 & 1.446 & 0.0023 & 0.0075 & 0.125 & 0.410 & 0.062 & 0.203 \\
\hline 28 & 0.153 & 0.577 & 0.495 & 1.866 & 0.0048 & 0.0181 & 0.104 & 0.392 & 0.143 & 0.539 \\
\hline 29 & 0.113 & 0.404 & 0.406 & 1.450 & 0.0028 & 0.0100 & 0.098 & 0.350 & 0. & 0.246 \\
\hline 30 & 0.134 & 0.393 & 0.561 & 1.646 & 0.0056 & 0.0164 & 0.161 & 0.472 & 36 & 0.282 \\
\hline 31 & 0.108 & 0.435 & 0.333 & 1.341 & 0.0044 & 0.0177 & 0.086 & 0.346 & & 09 \\
\hline 32 & 0.121 & 0.472 & 0.369 & 1.438 & 0.0028 & 0.0109 & 0.0 & 35 & 59 & 0.230 \\
\hline 33 & 0.109 & 0.445 & 0.329 & 1.342 & 0.0030 & 0.0122 & 0. & 0.371 & 40 & 0.163 \\
\hline 34 & 0.123 & 0.466 & 0.424 & 1.607 & 174 & 0.0 & & 0.375 & 39 & 0.148 \\
\hline 35 & 0.120 & 0.404 & 0.392 & 1.321 & 0.0270 & 0.0 & 0.074 & 0.249 & 0.046 & 0.155 \\
\hline 36 & 0.128 & 0.429 & 0.410 & 1.374 & 0.0034 & 0.0114 & 0.095 & 0.318 & 0.056 & 0.188 \\
\hline & 0.127 & 0.44 & 0.42 & 1.499 & 0.0052 & 0.0182 & 0.1 & 0.354 & 0.067 & 0.234 \\
\hline SED & .003 & 0.011 & 0.020 & 0.042 & 0.0015 & 0.0050 & 0.006 & 0.014 & 0.007 & 0.025 \\
\hline
\end{tabular}

Test Enclosure \#7, ALFCO \#4 Filter, 8 mice

\begin{tabular}{|c|c|c|c|c|c|c|c|c|c|c|}
\hline Mou & $\begin{array}{r}\text { e Heart } \\
\text { Wt }(g)\end{array}$ & $\begin{array}{l}\text { Heart } \\
\% \text { BW }\end{array}$ & $\begin{array}{l}\text { Kidney } \\
\text { Wt (g) }\end{array}$ & $\begin{array}{l}\text { Kidney } \\
\% \text { BW }\end{array}$ & $\begin{array}{l}\text { Adrenal } \\
\text { Wt (g) }\end{array}$ & $\begin{array}{l}\text { Adrenal } \\
\% \text { BW }\end{array}$ & $\begin{array}{l}\text { Spleen } \\
\text { Wt (g) }\end{array}$ & $\begin{array}{l}\text { Spleen } \\
\% \text { BW }\end{array}$ & $\begin{array}{l}\text { Thymus } \\
\text { Wt (g) }\end{array}$ & $\begin{array}{c}\text { Thymus } \\
\% \text { BW }\end{array}$ \\
\hline 37 & 0.110 & 0.432 & 0.373 & 1.463 & 0.0038 & 0.0149 & 0.089 & 0.349 & 0.049 & 0.192 \\
\hline 38 & 0.111 & 0.369 & 0.426 & 1.415 & 0.0044 & 0.0146 & 0.091 & 0.302 & 0.089 & 0.296 \\
\hline 39 & 0.136 & 0.468 & 0.443 & 1.525 & 0.0016 & 0.0055 & 0.133 & 0.458 & 0.066 & 0.227 \\
\hline 40 & 0.136 & 0.493 & 0.431 & 1.562 & 0.0029 & 0.0105 & 0.078 & 0.283 & 0.055 & 0.199 \\
\hline 41 & 0.120 & 0.458 & 0.395 & 1.506 & 0.0020 & 0.0076 & 0.099 & 0.378 & 0.073 & 0.278 \\
\hline 42 & 0.140 & 0.443 & 0.390 & 1.233 & 0.0035 & 0.0111 & 0.086 & 0.272 & 0.056 & 0.177 \\
\hline 43 & 0.126 & 0.414 & 0.406 & 1.333 & 0.0036 & 0.0118 & 0.086 & 0.282 & 0.079 & 0.259 \\
\hline 44 & 0.121 & 0.448 & 0.394 & 1.460 & 0.0042 & 0.0156 & 0.084 & 0.311 & 0.062 & 0.230 \\
\hline e & 0.125 & 0.440 & 0.407 & 1.437 & 0.0033 & 0.0115 & 0.093 & 0.329 & 0.066 & 0.232 \\
\hline & 0.004 & 0.013 & 0.008 & 0.038 & 0.0004 & 0.0013 & 0.006 & 0.022 & 0.005 & 0.015 \\
\hline
\end{tabular}


Test Enclosure \#1, ALFCO \#4 Filter, 12 mice

\begin{tabular}{|c|c|c|c|c|c|c|c|c|c|c|}
\hline $\begin{array}{l}\text { Mouse } \\
\text { id \# }\end{array}$ & $\begin{array}{l}\text { Heart } \\
\text { Wt (g) }\end{array}$ & $\begin{array}{l}\text { Heart } \\
\% \text { BW }\end{array}$ & $\begin{array}{l}\text { Kidney } \\
\text { Wt (g) }\end{array}$ & $\begin{array}{l}\text { Kidney } \\
\% \text { BW }\end{array}$ & $\begin{array}{l}\text { Adrenal } \\
\text { Wt (g) }\end{array}$ & $\begin{array}{l}\text { Adrenal } \\
\% \text { BW }\end{array}$ & $\begin{array}{l}\text { Spleen } \\
\text { Wt (g) }\end{array}$ & $\begin{array}{l}\text { Spleen } \\
\% \text { BW }\end{array}$ & $\begin{array}{l}\text { Thymus } \\
\text { Wt (g) }\end{array}$ & $\begin{array}{l}\text { Thymus } \\
\% \text { BW }\end{array}$ \\
\hline 45 & 0.104 & 0.382 & 0.370 & 1.361 & 0.0050 & 0.0184 & 0.094 & 0.346 & 0.080 & 0.294 \\
\hline 46 & 0.133 & 0.432 & 0.416 & 1.351 & 0.0047 & 0.0153 & 0.114 & 0.370 & 0.069 & 0.224 \\
\hline 47 & 0.141 & 0.518 & 0.436 & 1.601 & 0.0031 & 0.0114 & 0.095 & 0.349 & 0.077 & 0.283 \\
\hline 48 & 0.113 & 0.409 & 0.430 & 1.557 & 0.0022 & 0.0080 & 0.142 & 0.514 & 0.059 & 0.214 \\
\hline 49 & 0.121 & 0.489 & 0.435 & 1.758 & 0.0013 & 0.0053 & 0.091 & 0.368 & 0.038 & 0.154 \\
\hline 50 & 0.120 & 0.417 & 0.420 & 1.460 & 0.0053 & 0.0184 & 0.080 & 0.278 & 0.040 & 0.139 \\
\hline 51 & 0.130 & 0.478 & 0.386 & 1.418 & 0.0066 & 0.0242 & 0.073 & 0.268 & 0.046 & 0.169 \\
\hline 52 & 0.128 & 0.410 & 0.482 & 1.543 & 0.0041 & 0.0131 & 0.146 & 0.467 & 0.098 & 0.314 \\
\hline 53 & 0.139 & 0.444 & 0.469 & 1.498 & 0.0077 & 0.0246 & 0.111 & 0.355 & 0.076 & 0.243 \\
\hline 54 & 0.120 & 0.449 & 0.420 & 1.570 & 0.0039 & 0.0146 & 0.110 & 0.411 & 0.070 & 0.262 \\
\hline 55 & 0.148 & 0.484 & 0.465 & 1.522 & 0.0070 & 0.0229 & 0.095 & 0.311 & 0.050 & 0.164 \\
\hline 56 & 0.100 & 0.357 & 0.400 & 1.427 & 0.0043 & 0.0153 & 0.080 & 0.285 & 0.060 & 0.214 \\
\hline$M$ & 0.125 & 0.439 & 0.427 & 1.505 & 0.0046 & 0.0160 & 0.103 & 0.360 & 0.064 & 0.223 \\
\hline SEM & 0.004 & 0.014 & 0.0010 & 0.033 & 0.0005 & 0.0018 & 0.007 & 0.022 & 0.005 & 0.017 \\
\hline
\end{tabular}

Test Enclosure *2, ALFCO \#4 Filter, 16 mice

\begin{tabular}{|c|c|c|c|c|c|c|c|c|c|c|}
\hline $\begin{array}{l}\text { Mouse } \\
\text { id \# }\end{array}$ & $\begin{array}{l}\text { Heart } \\
\text { Wt }(\mathrm{g})\end{array}$ & $\begin{array}{l}\text { Heart } \\
\% \text { BW }\end{array}$ & $\begin{array}{l}\text { Kidney } \\
\text { Wt (g) }\end{array}$ & $\begin{array}{l}\text { Kidney } \\
\% \text { BW }\end{array}$ & $\begin{array}{l}\text { Adrenal } \\
\text { Wt }(g)\end{array}$ & $\begin{array}{l}\text { Adrenal } \\
\% \mathrm{BW}\end{array}$ & $\begin{array}{l}\text { Spleen } \\
\text { Wt (g) }\end{array}$ & $\begin{array}{l}\text { Spleen } \\
\% \text { BW }\end{array}$ & $\begin{array}{l}\text { Thymus } \\
\text { Wt (g) }\end{array}$ & $\begin{array}{l}\text { Thymus } \\
\% \text { BW }\end{array}$ \\
\hline 57 & 0.128 & 0.473 & 0.435 & 1.606 & 0.0025 & 0.0092 & 0.094 & 0.347 & 0.095 & 0.351 \\
\hline 58 & 0.126 & 0.462 & 0.388 & 1.423 & 0.0020 & 0.0073 & 0.090 & 0.330 & 0.046 & 0.169 \\
\hline 59 & 0.124 & 0.463 & 0.429 & 1.601 & 0.0035 & 0.0131 & 0.099 & 0.370 & 0.070 & 0.261 \\
\hline 60 & 0.136 & 0.464 & 0.432 & 1.475 & 0.0071 & 0.0242 & 0.093 & 0.318 & 0.064 & 0.219 \\
\hline 61 & 0.138 & 0.470 & 0.482 & 1.642 & 0.0034 & 0.0116 & 0.121 & 0.412 & 0.086 & 0.293 \\
\hline 62 & 0.113 & 0.415 & 0.389 & 1.427 & 0.0040 & 0.0147 & 0.095 & 0.348 & 0.087 & 0.319 \\
\hline 63 & 0.118 & 0.427 & 0.447 & 1.617 & 0.0030 & 0.0108 & 0.091 & 0.329 & 0.057 & 0.206 \\
\hline 64 & 0.112 & 0.391 & 0.415 & 1.448 & & & 0.149 & 0.520 & 0.115 & 0.401 \\
\hline 65 & 0.121 & 0.368 & 0.498 & 1.514 & 0.0035 & 0.0106 & 0.094 & 0.286 & 0.072 & 0.219 \\
\hline 66 & 0.114 & 0.400 & 0.385 & 1.352 & 0.0023 & 0.0081 & 0.085 & 0.299 & 0.098 & 0.344 \\
\hline 67 & 0.114 & 0.433 & 0.474 & 1.800 & 0.0040 & 0.0152 & 0.087 & 0.330 & 0.052 & 0.197 \\
\hline 68 & 0.112 & 0.427 & 0.425 & 1.621 & 0.0016 & 0.0061 & 0.084 & 0.320 & 0.083 & 0.317 \\
\hline 69 & 0.128 & 0.455 & 0.455 & 1.619 & 0.0035 & 0.0125 & 0.106 & 0.377 & 0.145 & 0.516 \\
\hline 70 & 0.125 & 0.473 & 0.329 & 1.245 & 0.0015 & 0.0057 & 0.099 & 0.375 & 0.086 & 0.326 \\
\hline 71 & 0.115 & 0.427 & 0.381 & 1.415 & 0.0047 & 0.0175 & 0.112 & 0.416 & 0.091 & 0.338 \\
\hline 72 & 0.119 & 0.381 & 0.439 & 1.404 & 0.0040 & 0.0128 & 0.108 & 0.345 & 0.120 & 0.384 \\
\hline Mean & 0.121 & 0.433 & 0.425 & 1.513 & 0.0034 & 0.0120 & 0.100 & 0.358 & 0.085 & 0.304 \\
\hline SEM & 0.002 & 0.009 & 0.011 & 0.035 & 0.0004 & 0.0012 & 0.004 & 0.014 & 0.007 & 0.023 \\
\hline
\end{tabular}




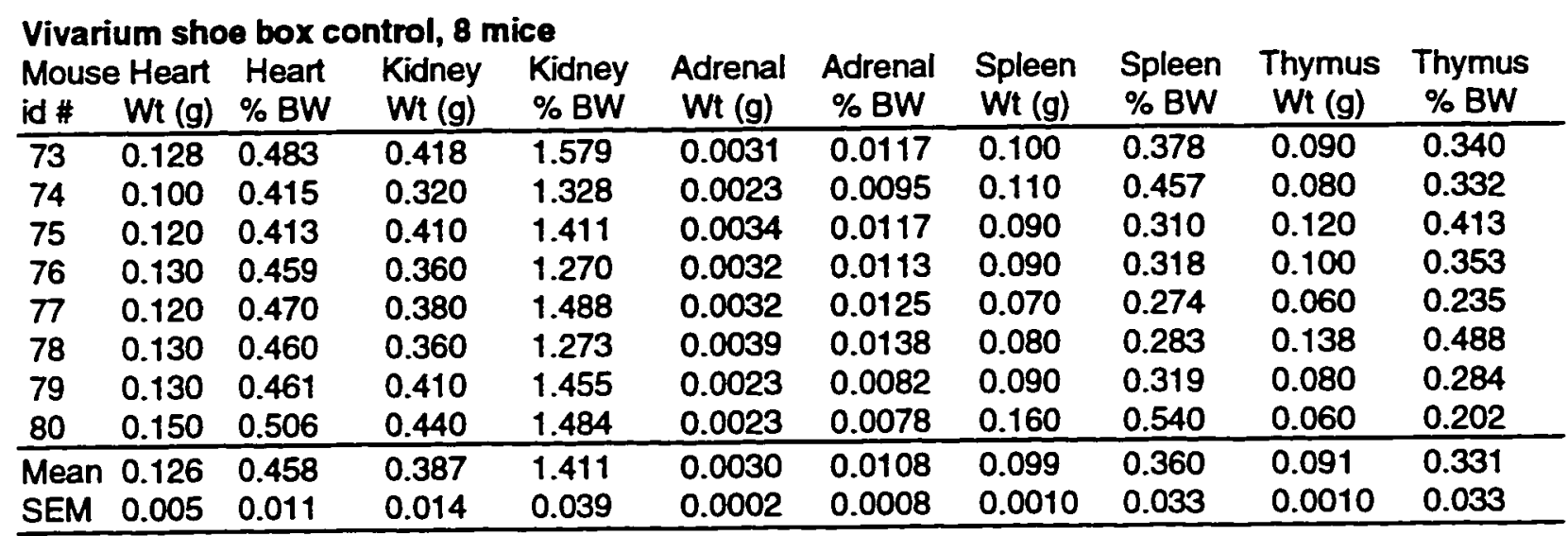

Vivarium Control Enclosure, 8 mice

\begin{tabular}{|c|c|c|c|c|c|c|c|c|c|c|}
\hline $\begin{array}{l}\text { Mouse } \\
\text { id \# }\end{array}$ & $\begin{array}{l}\text { Heart } \\
\text { Wt }(g)\end{array}$ & $\begin{array}{l}\text { Heart } \\
\% \text { BW }\end{array}$ & $\begin{array}{l}\text { Kidney } \\
\text { Wt (g) }\end{array}$ & $\begin{array}{l}\text { Kidney } \\
\% \text { BW }\end{array}$ & $\begin{array}{l}\text { Adrenal } \\
\text { Wt }(g)\end{array}$ & $\begin{array}{l}\text { Adrenal } \\
\% \text { BW }\end{array}$ & $\begin{array}{l}\text { Spleen } \\
\text { Wt (g) }\end{array}$ & $\begin{array}{l}\text { Spleen } \\
\% \text { BW }\end{array}$ & $\begin{array}{l}\text { Thymus } \\
\text { Wt (g) }\end{array}$ & $\begin{array}{l}\text { Thymus } \\
\% \text { BW }\end{array}$ \\
\hline 81 & 0.122 & 0.467 & 0.388 & 1.484 & 0.0025 & 0.0096 & 0.105 & 0.402 & 0.034 & 0.130 \\
\hline 82 & 0.140 & 0.500 & 0.420 & 1.501 & 0.0025 & 0.0089 & 0.097 & 0.347 & 0.050 & 0.179 \\
\hline 83 & 0.163 & 0.420 & 0.543 & 1.399 & 0.0009 & 0.0023 & 0.152 & 0.392 & 0.086 & 0.222 \\
\hline 84 & 0.153 & 0.524 & 0.426 & 1.458 & 0.0045 & 0.0154 & 0.075 & 0.257 & 0.073 & 0.250 \\
\hline 85 & 0.125 & 0.448 & 0.428 & 1.534 & 0.0050 & 0.0179 & 0.092 & 0.330 & 0.068 & 0.244 \\
\hline 86 & 0.148 & 0.500 & 0.375 & 1.266 & 0.0013 & 0.0044 & 0.090 & 0.304 & 0.068 & 0.230 \\
\hline 87 & 0.148 & 0.488 & 0.405 & 1.336 & 0.0047 & 0.0155 & 0.089 & 0.294 & 0.092 & 0.303 \\
\hline 88 & 0.125 & 0.451 & 0.399 & 1.440 & 0.0023 & 0.0083 & 0.125 & 0.451 & 0.070 & 0.253 \\
\hline & 0.141 & 0.475 & 0.423 & 1.427 & 0.0030 & 0.0103 & 0.103 & 0.347 & 0.068 & 0.226 \\
\hline$-a$ & 0.005 & 0.012 & 0.018 & 0.032 & 0.0006 & 0.0020 & 0.009 & 0.023 & 0.007 & 0.018 \\
\hline
\end{tabular}


Table 17 (Experiment 1). Cage mean \pm S.E.M for organ weights in grams and as a percentage of body weight (BW).

\begin{tabular}{|c|c|c|c|c|}
\hline & $\begin{array}{l}\text { Heart } \\
\text { Wt }(g)\end{array}$ & $\begin{array}{l}\text { Heart } \\
\% \text { BW }\end{array}$ & $\begin{array}{l}\text { Kidney } \\
\text { Wt (g) }\end{array}$ & $\begin{array}{l}\text { Kidney } \\
\% \text { BW } \\
\end{array}$ \\
\hline Vivarium Shoe Box Control, 8 mice & $0.126 \pm 0.005$ & $0.458 \pm 0.011$ & $0.387 \pm 0.014$ & $1.411 \pm 0.039$ \\
\hline Vivarium Control Enclosure, 8 mice & $0.141 \pm 0.005$ & $0.475 \pm 0.012$ & $0.423 \pm 0.018$ & $1.427 \pm 0.032$ \\
\hline Test enclosure \#3, ALFCO \#3A Filter, 8 mice & $0.121 \pm 0.003$ & $0.406 \pm 0.013$ & $0.389 \pm 0.013$ & $1.311 \pm 0.060$ \\
\hline Test enclosure $\# 7$, ALFCO \#4 Filter, 8 mice & $0.125 \pm 0.004$ & $0.440 \pm 0.013$ & $0.407 \pm 0.008$ & $1.437 \pm 0.038$ \\
\hline Test enclosure "6. ALFCO *3A Filter, 12 mice & $0.131 \pm 0.004$ & $0.450 \pm 0.011$ & $0.424 \pm 0.019$ & $1.451 \pm 0.053$ \\
\hline Test enclosure "1, ALFCO \#4 Filter, 12 mice & $0.125 \pm 0.004$ & $0.439 \pm 0.014$ & $0.427 \pm 0.001$ & $1.505 \pm 0.033$ \\
\hline Test enclosure "5, ALFCO "3A Filter. 16 mice & $0.127 \pm 0.003$ & $0.447 \pm 0.011$ & $0.429 \pm 0.020$ & $1.499 \pm 0.042$ \\
\hline Test enclosure *2. ALFCO \#4 Filter, 16 mice & $0.121 \pm 0.002$ & $0.433 \pm 0.009$ & $0.425 \pm 0.011$ & $1.513 \pm 0.035$ \\
\hline
\end{tabular}

\begin{tabular}{|c|c|c|c|c|}
\hline & $\begin{array}{l}\text { Adrenal } \\
\text { Wt (mg) }\end{array}$ & $\begin{array}{c}\text { Adrenal } \\
\% \mathrm{BW} \times 10^{-4} \\
\end{array}$ & $\begin{array}{l}\text { Spleen } \\
\text { Wt (g)\% BW }\end{array}$ & Spleen \\
\hline Vivarium Shoo Box Control, 8 mice & $3.0 \pm 0.2$ & $10.8 \pm 0.8$ & $0.099 \pm 0.001$ & $0.360 \pm 0.033$ \\
\hline Vivarium Control Enclosure, 8 mice & $3.0 \pm 0.6$ & $10.3 \pm 2.0$ & $0.103 \pm 0.009$ & $0.347 \pm 0.023$ \\
\hline Test enclosure \#3. ALFCO \#3A Filter, 8 mice & $3.1 \pm 0.5$ & $10.7 \pm 1.8$ & $0.091 \pm 0.008$ & $0.309 \pm 0.032$ \\
\hline Test enclosure "7, ALFCO \#4 Filter, 8 mice & $3.3 \pm 0.4$ & $11.5 \pm 1.3$ & $0.093 \pm 0.006$ & $0.329 \pm 0.022$ \\
\hline Test enclosure "6. ALFCO "3A Filter, 12 mice & $3.4 \pm 0.5$ & $11.5 \pm 1.8$ & $0.104 \pm 0.005$ & $0.354 \pm 0.011$ \\
\hline Test enclosure "1. ALFCO \#4 Filter. 12 mice & $4.6 \pm 0.5$ & $16.0 \pm 1.8$ & $0.103 \pm 0.007$ & $0.360 \pm 0.022$ \\
\hline Test enclosure \#5. ALFCO \#3A Filter, 16 mice & $5.2 \pm 1.5$ & $18.2 \pm 5.0$ & $0.101 \pm 0.006$ & $0.354 \pm 0.014$ \\
\hline Test enclosure \#2, ALFCO \#4 Filter. 16 mice & $3.4 \pm 0.4$ & $12.0 \pm 1.2$ & $0.100 \pm 0.004$ & $0.358 \pm 0.014$ \\
\hline
\end{tabular}

\begin{tabular}{lcc} 
& $\begin{array}{c}\text { Thymus } \\
\text { Wt }(\mathrm{g})\end{array}$ & $\begin{array}{c}\text { Thymus } \\
\% \mathrm{BW}\end{array}$ \\
\hline Vivarium Shoe Box Control, 8 mice & $0.091 \pm 0.001$ & $0.331 \pm 0.033$ \\
Vivarium Control Enclosure, 8 mice & $0.068 \pm 0.007$ & $0.226 \pm 0.018$ \\
Test enclosure \#3. ALFCO \#3A Filter, 8 mice & $0.076 \pm 0.010$ & $0.259 \pm 0.037$ \\
Test enclosure \#7. ALFCO \#4 Filter, 8 mice & $0.066 \pm 0.005$ & $0.232 \pm 0.015$ \\
Test enclosure \#6. ALFCO \#3A Filter, 12 mice & $0.081 \pm 0.005$ & $0.277 \pm 0.016$ \\
Test enclosure \#1. ALFCO \#4 Filter, 12 mice & $0.064 \pm 0.005$ & $0.223 \pm 0.017$ \\
Test enclosure \#5. ALFCO \#3A Filter. 16 mice & $0.067 \pm 0.007$ & $0.234 \pm 0.025$ \\
Test enclosure \#2. ALFCO \#4 Filter, 16 mice & $0.085 \pm 0.007$ & $0.304 \pm 0.023$
\end{tabular}


Figure 15 (Experiment 1). Mean \pm S.E.M. heart weight. and mean \pm S.E.M. heart weight as a percent of final body weight. (See also Table 17).
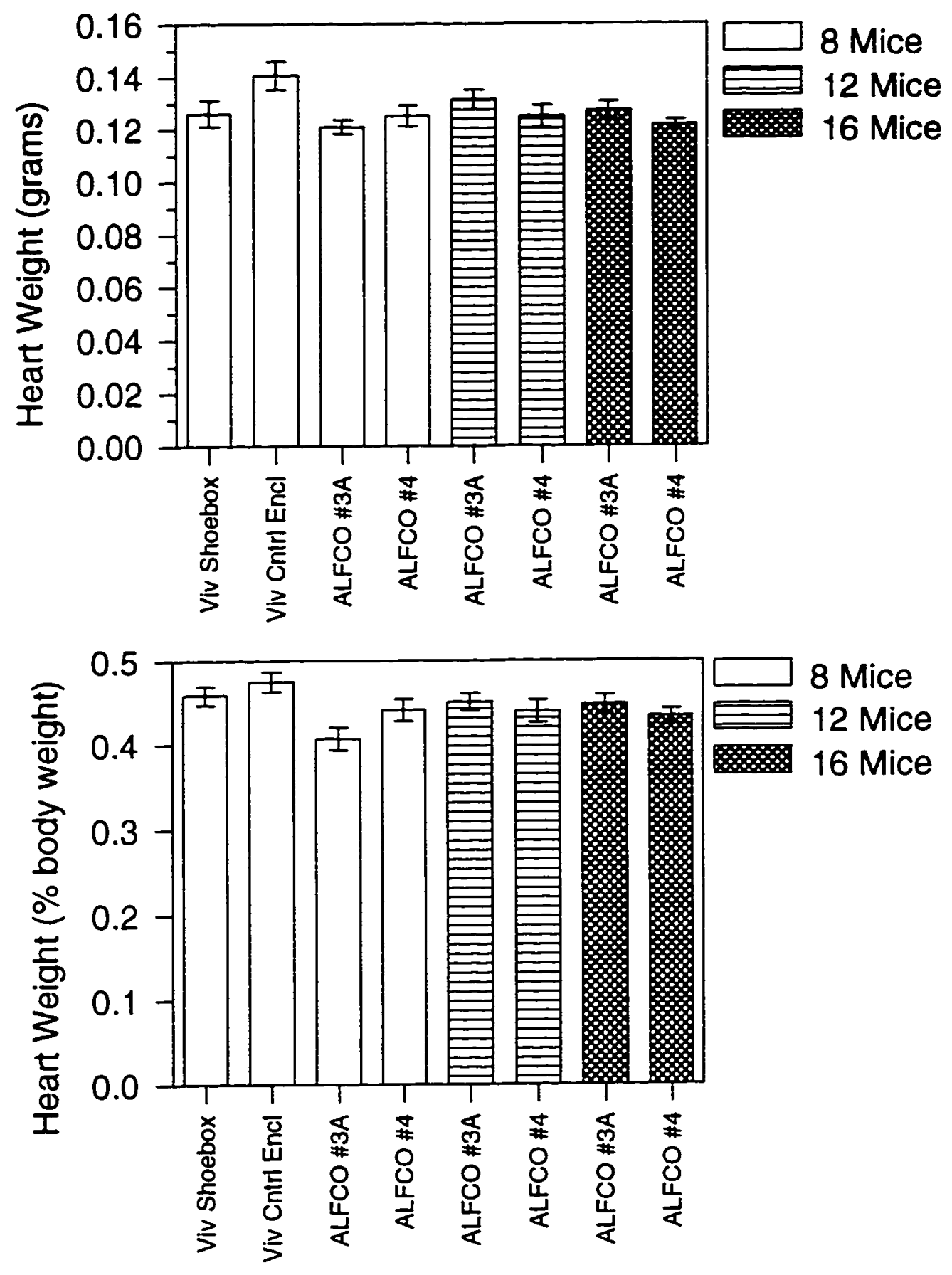
Figure 16 (Experiment 1). Mean \pm S.E.M. kidney weight. and mean \pm S.E.M. kidney weight as a percent of final body weight. (See also Table 17).
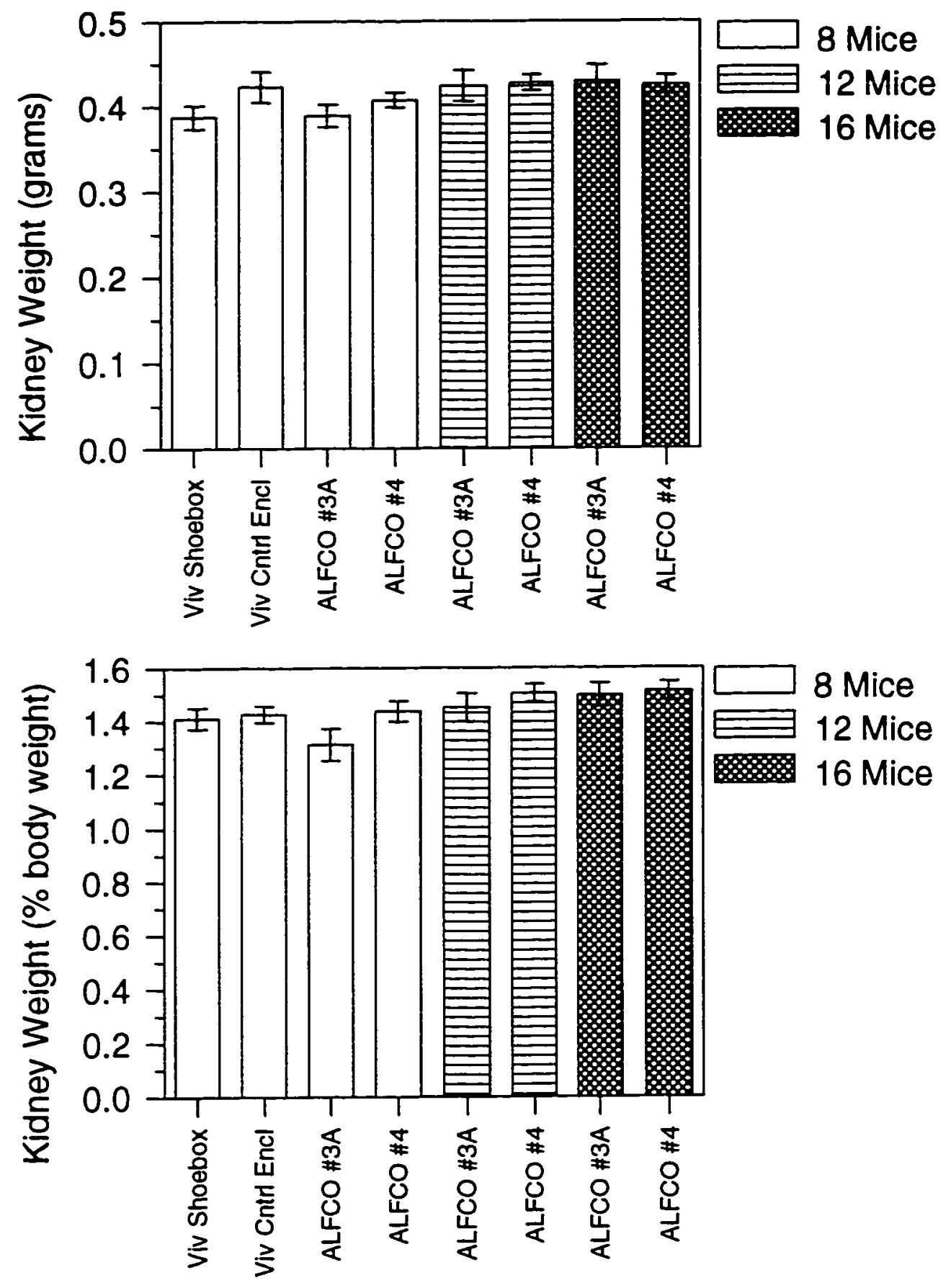
Figure 17 (Experiment 1). Mean \pm S.E.M. bilateral adrenal weight. and mean \pm S.E.M. bilateral adrenal weight as a percent of final body weight. (See also Table 17).
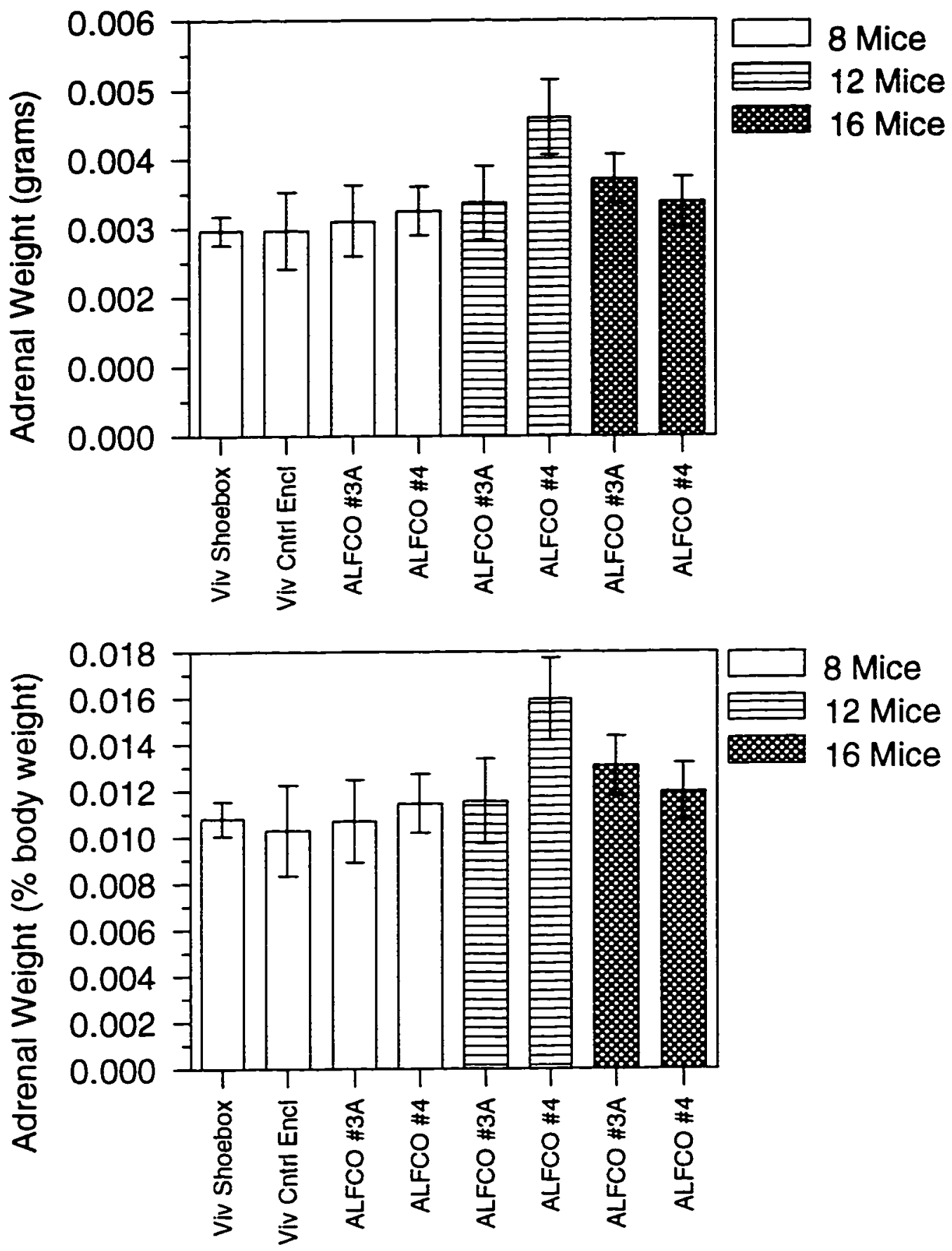
Figure 18 (Experiment 1). Mean \pm S.E.M. spleen weight. and mean \pm S.E.M. spleen weight as a percent of final body weight. (See also Table 17).
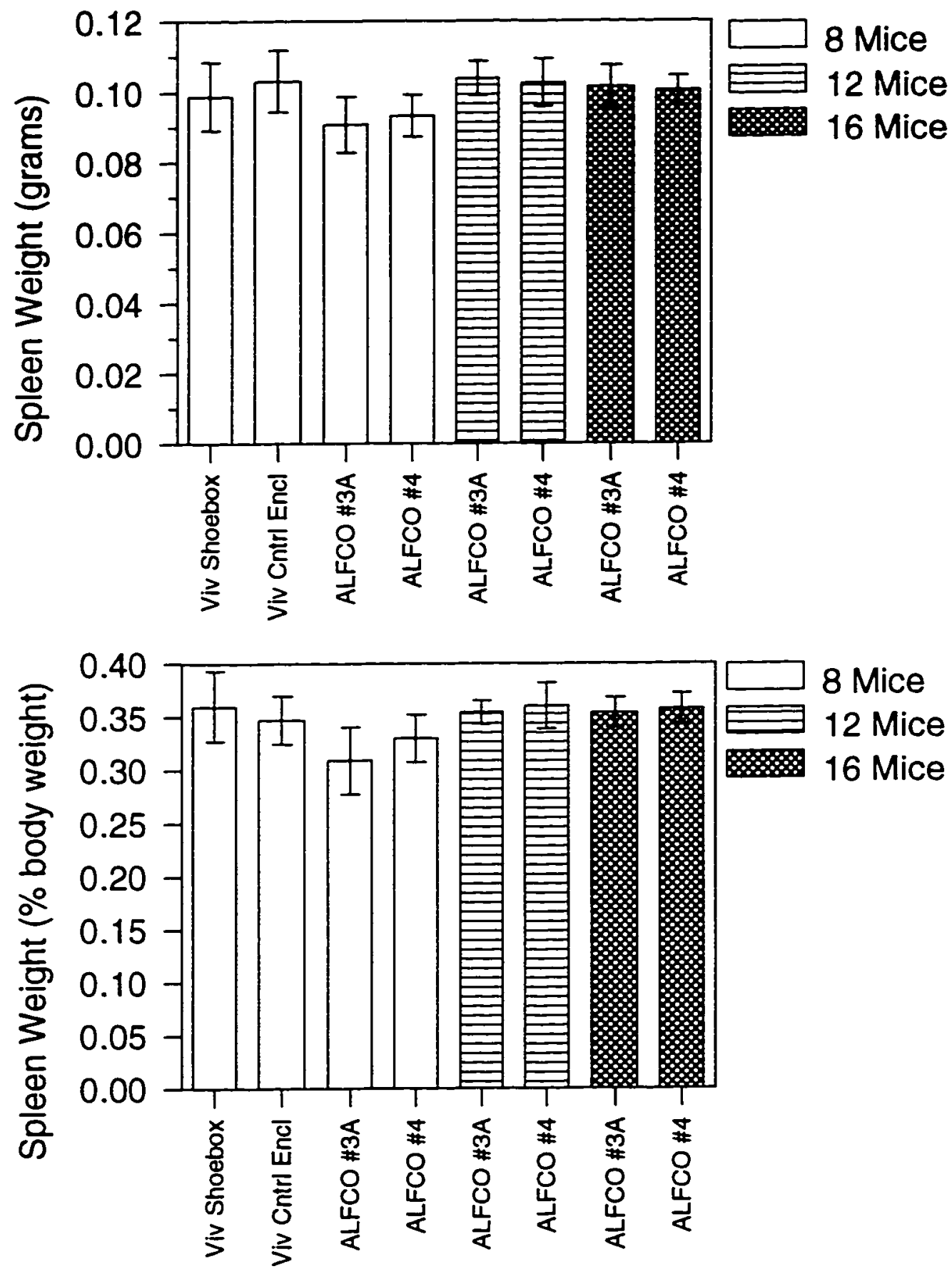
Figure 19 (Experiment 1). Mean \pm S.E.M. thymus weight, and mean \pm S.E.M. thymus weight as a percent of final body weight. (See also Table 17).
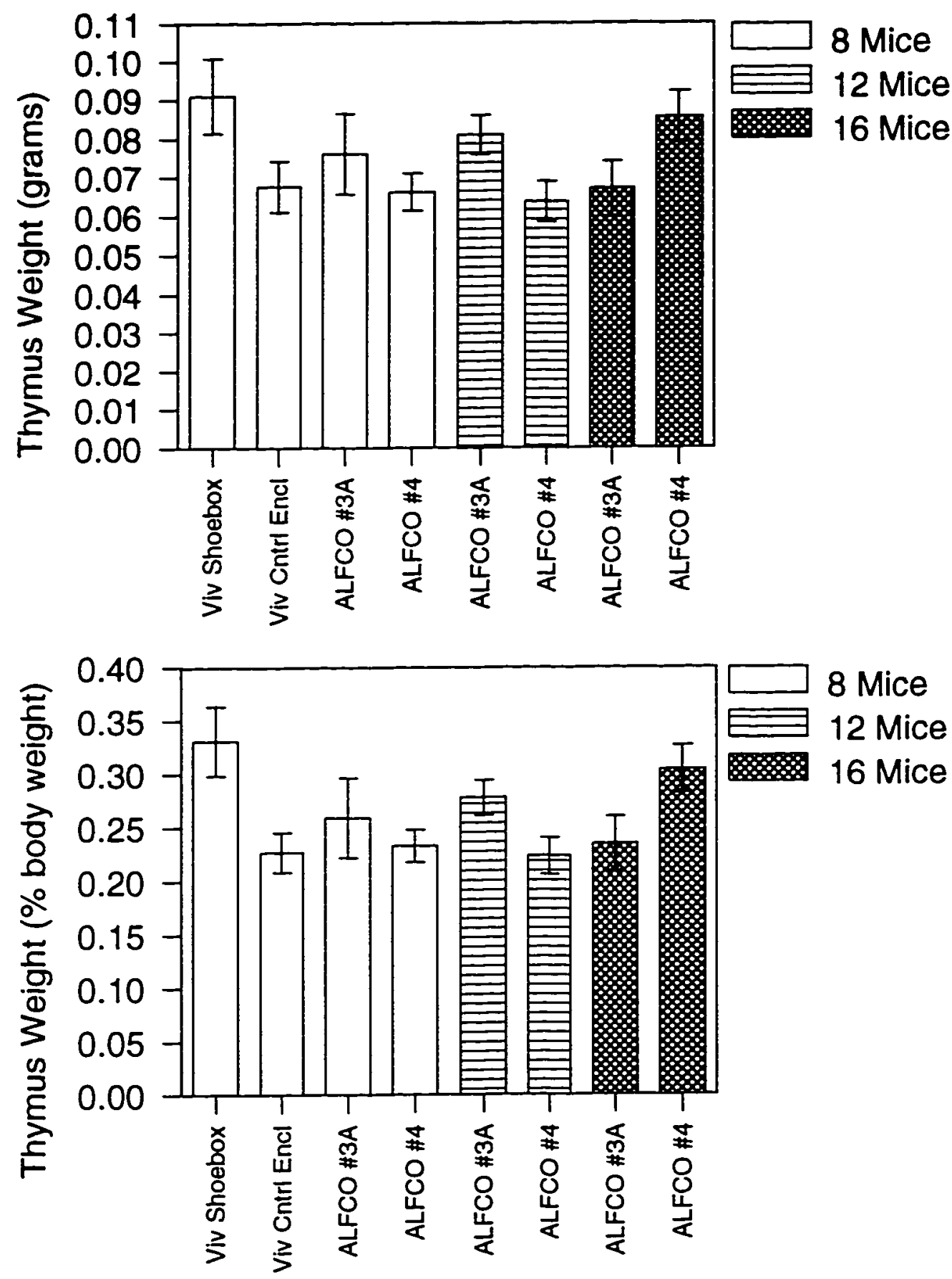
Table 18 (Experiment 1). Total white blood cell count (WBC), plasma corticosterone, plasma protein, eosinophil (EOS) count, basophil (BAS) count, neutrophil (NEU) count, monocyte (MON) count, lymphocyte (LYM) count, and neutrophil to lymphocyte ratio (N/L).

\begin{tabular}{|c|c|c|c|c|c|c|c|c|c|}
\hline $\begin{array}{l}\text { Mouse } \\
\text { id \# }\end{array}$ & $\begin{array}{c}\text { Plasma } \\
\text { Corticosterone } \\
\mu g / d l\end{array}$ & $\begin{array}{c}\text { Plasma } \\
\text { Protein } \\
\text { g/dl }\end{array}$ & $\begin{array}{c}\text { Total } \\
\text { WBC } \\
\text { cells } \times 10^{3} / \mathrm{ul}\end{array}$ & $\begin{array}{c}\text { EOS } \\
\text { count } \\
\% \text { WBC }\end{array}$ & $\begin{array}{c}\text { BAS } \\
\text { count } \\
\% \text { WBC }\end{array}$ & $\begin{array}{c}\text { NEU } \\
\text { count } \\
\% \text { WBC }\end{array}$ & $\begin{array}{c}\text { MON } \\
\text { count } \\
\% \text { WBC }\end{array}$ & $\begin{array}{c}\text { LYM } \\
\text { count } \\
\% \text { WBC }\end{array}$ & $\begin{array}{l}\mathrm{N} / \mathrm{L} \\
\text { ratio }\end{array}$ \\
\hline$\overline{1}$ & 4.36 & 4.07 & 10.38 & 0 & 0 & 2 & 3 & 95 & 0.021 \\
\hline 2 & 2.52 & 4.50 & & & & & & & \\
\hline 3 & 1.90 & 6.24 & 7.88 & 5 & 0 & 7 & 9 & 79 & 0.089 \\
\hline 4 & 10.48 & 5.25 & 5.00 & 4 & 0 & 9 & 3 & 84 & 0.107 \\
\hline 5 & 0.19 & 3.85 & 15.38 & 0 & 0 & 0 & 1 & 99 & 0.000 \\
\hline 6 & 1.88 & 3.50 & 10.63 & 0 & 0 & 3 & 3 & 94 & 0.032 \\
\hline 7 & 1.52 & 3.98 & 9.38 & 2 & 0 & 2 & 0 & 96 & 0.021 \\
\hline 8 & 2.52 & 3.85 & 14.25 & 0 & 0 & 9 & 9 & 82 & 0.110 \\
\hline$\overline{\text { Mean }}$ & 3.17 & 4.41 & 10.41 & 1.6 & 0.0 & 4.6 & 4.0 & 90 & 0.054 \\
\hline S.E.M & 1.12 & 0.32 & 1.35 & 0.8 & 0.0 & 1.4 & 1.4 & 3 & 0.017 \\
\hline
\end{tabular}

\begin{tabular}{|c|c|c|c|c|c|c|c|c|c|}
\hline $\begin{array}{l}\text { Mouse } \\
\text { id \# }\end{array}$ & $\begin{array}{c}\text { Plasma } \\
\text { Conticosterone } \\
\mu g / d l\end{array}$ & $\begin{array}{l}\text { Plasma } \\
\text { Protein } \\
g / d l\end{array}$ & $\begin{array}{c}\text { Total } \\
\text { WBC } \\
\text { cells } \times 10^{3} / \mathrm{ul}\end{array}$ & $\begin{array}{c}\text { EOS } \\
\text { count } \\
\% \text { WBC }\end{array}$ & $\begin{array}{c}\text { BAS } \\
\text { count } \\
\% \text { WBC }\end{array}$ & $\begin{array}{c}\text { NEU } \\
\text { count } \\
\% \text { WBC }\end{array}$ & $\begin{array}{c}\text { MON } \\
\text { count } \\
\% \text { WBC } \\
\end{array}$ & $\begin{array}{c}\text { LYM } \\
\text { count } \\
\% \text { WBC } \\
\end{array}$ & $\begin{array}{l}\mathrm{N} / \mathrm{L} \\
\text { ratio }\end{array}$ \\
\hline$\overline{9}$ & 12.22 & 2.99 & 11.38 & 1 & 0 & $\overline{0}$ & 2 & 97 & 0.000 \\
\hline 10 & 21.85 & 2.74 & 7.50 & 3 & 0 & 3 & 4 & 90 & 0.033 \\
\hline 11 & 19.55 & 3.16 & 10.25 & 0 & 0 & 4 & 0 & 96 & 0.042 \\
\hline 12 & 3.95 & 2.96 & 4.50 & 2 & 2 & 3 & 4 & 91 & 0.033 \\
\hline 13 & & & 2.00 & 6 & 0 & 9 & 2 & 83 & 0.108 \\
\hline 14 & 9.17 & 2.65 & 3.63 & 3 & 0 & 7 & 4 & 86 & 0.081 \\
\hline 15 & 15.74 & 3.98 & 9.13 & 2 & 0 & 9 & 3 & 86 & 0.105 \\
\hline 16 & 22.69 & 3.98 & 13.63 & 0 & 0 & 1 & 3 & 96 & 0.010 \\
\hline 17 & 12.12 & 2.54 & 4.38 & 1 & 0 & 3 & 1 & 95 & 0.032 \\
\hline 18 & 31.20 & 3.27 & 11.75 & 1 & 0 & 8 & 4 & 87 & 0.092 \\
\hline 19 & 3.41 & 0.04 & 15.75 & 2 & 0 & 4 & 2 & 92 & 0.043 \\
\hline 20 & 7.48 & 1.02 & 4.00 & 10 & 0 & 3 & 5 & 82 & 0.037 \\
\hline Mean & 14.49 & 2.67 & 8.16 & 2.6 & 0.2 & 4.5 & 2.8 & 90.1 & 0.051 \\
\hline S.E.M & 2.61 & 0.36 & 1.29 & 0.8 & 0.2 & 0.9 & 0.4 & 1.5 & 0.010 \\
\hline
\end{tabular}




\begin{tabular}{|c|c|c|c|c|c|c|c|c|c|}
\hline $\begin{array}{l}\text { Mouse } \\
\text { id \# }\end{array}$ & $\begin{array}{c}\text { Plasma } \\
\text { Corticosterone } \\
\mu g / d l\end{array}$ & $\begin{array}{c}\text { Plasma } \\
\text { Protein } \\
\text { g/dl } \\
\end{array}$ & $\begin{array}{c}\text { Total } \\
\text { WBC } \\
\text { cells } \times 10^{3} / \text { ul }\end{array}$ & $\begin{array}{c}\text { EOS } \\
\text { count } \\
\% \text { WBC } \\
\end{array}$ & $\begin{array}{c}\text { BAS } \\
\text { count } \\
\% \text { WBC } \\
\end{array}$ & $\begin{array}{c}\text { NEU } \\
\text { count } \\
\% \text { WBC } \\
\end{array}$ & $\begin{array}{r}\text { MON } \\
\text { count } \\
\% \text { WBC } \\
\end{array}$ & $\begin{array}{c}\text { LYM } \\
\text { count } \\
\% \text { WBC } \\
\end{array}$ & $\begin{array}{l}N / 2 \\
\text { ratio }\end{array}$ \\
\hline$\overline{21}$ & 26.65 & 4.34 & 4.88 & 2 & 0 & 5 & 4 & 89 & 0.056 \\
\hline 22 & 24.76 & & 5.00 & 1 & 0 & 5 & 2 & 92 & 0.054 \\
\hline 23 & 25.45 & & 5.13 & & & & & & \\
\hline 24 & 22.11 & & 16.38 & 2 & 0 & 1 & 2 & 95 & 0.011 \\
\hline 25 & 23.22 & & 11.63 & 3 & 0 & 0 & 1 & 96 & 0.000 \\
\hline 26 & 15.50 & 2.10 & 3.13 & 3 & 0 & 3 & 1 & 93 & 0.032 \\
\hline 27 & 5.49 & & 4.63 & 2 & 0 & 8 & 3 & 87 & 0.092 \\
\hline 28 & 30.85 & 4.08 & 5.75 & 5 & 0 & 5 & 1 & 89 & 0.056 \\
\hline 29 & 17.30 & & & & & & & & \\
\hline 30 & 38.06 & 2.99 & 24.38 & 1 & 0 & 3 & 1 & 95 & 0.032 \\
\hline 31 & 37.17 & 3.73 & 4.50 & 2 & 0 & 4 & 1 & 93 & 0.043 \\
\hline 32 & 8.79 & 3.78 & 9.63 & 2 & 0 & 0 & 1 & 97 & 0.000 \\
\hline 33 & 18.29 & 1.49 & 31.63 & 2 & 0 & 2 & 2 & 94 & 0.021 \\
\hline 34 & 31.04 & 4.69 & 5.13 & 4 & 0 & 12 & 3 & 81 & 0.148 \\
\hline 35 & 13.75 & 3.34 & 7.25 & 1 & 0 & 2 & 3 & 94 & 0.021 \\
\hline 36 & 11.94 & 4.30 & 5.25 & 2 & 0 & 12 & 4 & 82 & 0.146 \\
\hline$\overline{\text { Mean }}$ & 21.90 & 3.48 & 9.62 & 2.3 & 0.0 & 4.4 & 2.1 & 91.2 & 0.051 \\
\hline S.E.M & 2.40 & 0.33 & 2.15 & 0.3 & 0.0 & 1.0 & 0.3 & 1.3 & 0.013 \\
\hline
\end{tabular}

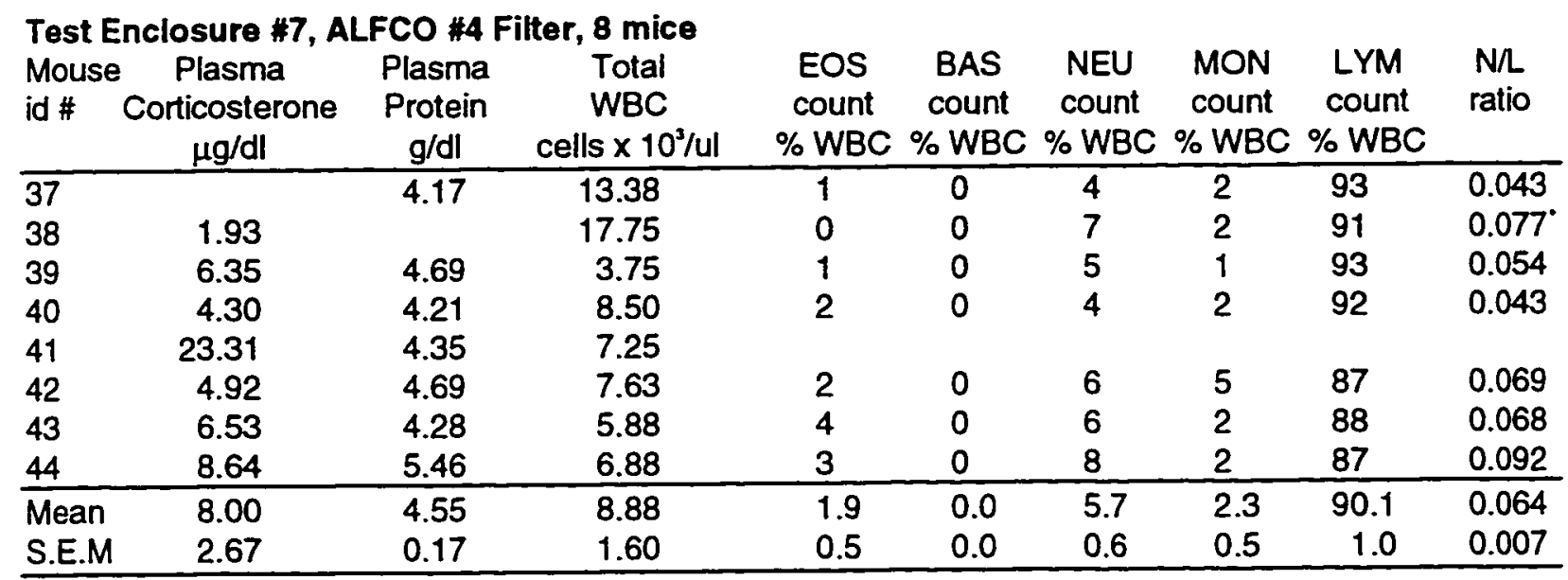




\begin{tabular}{|c|c|c|c|c|c|c|c|c|c|}
\hline $\begin{array}{l}\text { Mouse } \\
\text { id \# }\end{array}$ & $\begin{array}{c}\text { Plasma } \\
\text { Corticosterone } \\
\mu g / d l\end{array}$ & $\begin{array}{c}\text { Plasma } \\
\text { Protein } \\
g / d l\end{array}$ & $\begin{array}{c}\text { Total } \\
\text { WBC } \\
\text { cells } \times 10^{3} / \mathrm{ul}\end{array}$ & $\begin{array}{c}\text { EOS } \\
\text { count } \\
\% \text { WBC }\end{array}$ & $\begin{array}{c}\text { BAS } \\
\text { count } \\
\% \text { WBC }\end{array}$ & $\begin{array}{c}\text { NEU } \\
\text { count } \\
\% \text { WBC }\end{array}$ & $\begin{array}{c}\text { MON } \\
\text { count } \\
\% \text { WBC }\end{array}$ & $\begin{array}{c}\text { LYM } \\
\text { count } \\
\% \text { WBC }\end{array}$ & $\begin{array}{l}\mathrm{N} / \\
\text { ratio }\end{array}$ \\
\hline$\overline{45}$ & 14.82 & & 22.50 & 1 & 0 & 1 & 2 & 96 & 0.010 \\
\hline 46 & 3.52 & 4.99 & 8.88 & 2 & 0 & 10 & 3 & 85 & 0.118 \\
\hline 47 & 10.24 & 4.82 & 11.00 & 2 & 0 & 4 & 2 & 92 & 0.043 \\
\hline 48 & & 4.04 & 10.88 & 2 & 0 & 7 & 5 & 84 & 0.083 \\
\hline 49 & 8.34 & 7.42 & 9.75 & 3 & 0 & 0 & 3 & 94 & 0.000 \\
\hline 50 & 4.46 & 5.42 & 13.13 & 2 & 0 & 9 & 3 & 86 & 0.105 \\
\hline 51 & & & 7.38 & 4 & 0 & 9 & 3 & 84 & 0.107 \\
\hline 52 & 4.76 & 5.77 & 2.75 & 0 & 0 & 0 & 5 & 95 & 0.000 \\
\hline 53 & 4.33 & 2.99 & 6.63 & 2 & 0 & 5 & 7 & 86 & 0.058 \\
\hline 54 & 2.99 & 3.72 & 10.50 & 4 & 0 & 5 & 4 & 87 & 0.057 \\
\hline 55 & 11.75 & 4.50 & 9.13 & 0 & 0 & 7 & 6 & 87 & 0.080 \\
\hline 56 & 26.80 & 5.64 & 2.88 & 1 & 0 & 5 & 5 & 89 & 0.056 \\
\hline Mean & 9.20 & 4.93 & 9.61 & 1.9 & 0.0 & 5.2 & 4.0 & 88.8 & 0.060 \\
\hline S.E.M & 2.32 & 0.39 & 1.48 & 0.4 & 0.0 & 1.0 & 0.5 & 1.3 & 0.012 \\
\hline
\end{tabular}

\begin{tabular}{|c|c|c|c|c|c|c|c|c|c|}
\hline $\begin{array}{l}\text { Test E } \\
\text { Mouse } \\
\text { id \# }\end{array}$ & $\begin{array}{c}\text { Plasma } \\
\text { Corticosterone } \\
\mu \mathrm{g} / \mathrm{dl}\end{array}$ & $\begin{array}{c}\text { Plasma } \\
\text { Protein } \\
\mathrm{g} / \mathrm{dl}\end{array}$ & $\begin{array}{c}\text { Total } \\
\text { WBC } \\
\text { cells } \times 10^{3} / \mathrm{ul}\end{array}$ & $\begin{array}{c}\text { EOS } \\
\text { count } \\
\% \text { WBC }\end{array}$ & $\begin{array}{c}\text { BAS } \\
\text { count } \\
\% \text { WBC }\end{array}$ & $\begin{array}{c}\text { NEU } \\
\text { count } \\
\% \text { WBC } \\
\end{array}$ & $\begin{array}{c}\text { MON } \\
\text { count } \\
\% \text { WBC }\end{array}$ & $\begin{array}{c}\text { LYM } \\
\text { count } \\
\% \text { WBC } \\
\end{array}$ & $\begin{array}{l}N / L \\
\text { ratio }\end{array}$ \\
\hline$\overline{57}$ & 24.44 & 5.94 & 10.88 & 2 & 0 & 7 & 3 & 88 & 0.080 \\
\hline 58 & 9.32 & 5.65 & 17.50 & 0 & 0 & 2 & 2 & 96 & 0.021 \\
\hline 59 & 10.21 & 3.17 & 3.25 & 3 & 0 & 2 & 0 & 95 & 0.021 \\
\hline 60 & 15.82 & 2.24 & 7.00 & 0 & 0 & 3 & 1 & 96 & 0.031 \\
\hline 61 & 20.56 & 5.68 & 5.13 & 1 & 0 & 7 & 3 & 89 & 0.079 \\
\hline 62 & 31.41 & 4.34 & 7.63 & 0 & 0 & 4 & 2 & 94 & 0.043 \\
\hline 63 & 13.88 & 4.69 & 5.63 & 1 & 0 & 2 & 3 & 94 & 0.021 \\
\hline 64 & 8.74 & 5.51 & & & & & & & \\
\hline 65 & & & 7.63 & 0 & 0 & 2 & 5 & 93 & 0.022 \\
\hline 66 & 8.24 & 3.56 & 8.50 & 1 & 0 & 3 & 1 & 95 & 0.032 \\
\hline 67 & 14.14 & 3.86 & 10.00 & 2 & 0 & 5 & 0 & 93 & 0.054 \\
\hline 68 & 21.33 & 1.31 & 13.38 & 1 & 0 & 0 & 0 & 99 & 0.000 \\
\hline 69 & & & 31.75 & 3 & 0 & 2 & 4 & 91 & 0.022 \\
\hline 70 & 35.45 & 5.69 & 4.13 & 4 & 0 & 13 & 2 & 81 & 0.160 \\
\hline 71 & 17.12 & 4.91 & 8.25 & 5 & 0 & 10 & 2 & 83 & 0.120 \\
\hline 72 & 13.00 & 5.00 & 12.38 & 2 & 0 & 8 & 2 & 88 & 0.091 \\
\hline$\overline{\mathrm{Me}}$ & 17.40 & 4.40 & 10.20 & 1.7 & 0.0 & 4.7 & 2.0 & 91.7 & 0.053 \\
\hline S.E.I & 2.25 & 0.38 & 1.82 & 0.4 & 0.0 & 0.9 & 0.4 & 1.3 & 0.012 \\
\hline
\end{tabular}




\begin{tabular}{|c|c|c|c|c|c|c|c|c|c|}
\hline $\begin{array}{l}\text { Vivaric } \\
\text { Mouse } \\
\text { id \# }\end{array}$ & $\begin{array}{l}\text { um shoe box c } \\
\text { Plasma } \\
\text { Corticosterone } \\
\mu g / d l\end{array}$ & $\begin{array}{c}\text { ntrol, } 8 \text { n } \\
\text { Plasma } \\
\text { Protein } \\
\text { g/dl }\end{array}$ & $\begin{array}{c}\text { Total } \\
\text { WBC } \\
\text { cells } \times 10^{3} / \mathrm{ul}\end{array}$ & $\begin{array}{c}\text { EOS } \\
\text { count } \\
\% \text { WBC }\end{array}$ & $\begin{array}{c}\text { BAS } \\
\text { count } \\
\% \text { WBC }\end{array}$ & $\begin{array}{c}\text { NEU } \\
\text { count } \\
\% \text { WBC } \\
\end{array}$ & $\begin{array}{c}\text { MON } \\
\text { count } \\
\% \text { WBC } \\
\end{array}$ & $\begin{array}{c}\text { LYM } \\
\text { count } \\
\% \text { WBC } \\
\end{array}$ & $\begin{array}{c}N / L \\
\text { count }\end{array}$ \\
\hline$\overline{73}$ & 4.82 & 3.71 & 6.50 & 0 & $\overline{0}$ & 1 & 4 & 95 & 0.011 \\
\hline 74 & 3.33 & 3.62 & 4.88 & 1 & 0 & 1 & 1 & 97 & 0.010 \\
\hline 75 & 7.16 & 4.52 & 12.13 & 3 & 0 & 10 & 3 & 84 & 0.119 \\
\hline 76 & 3.35 & 4.73 & 11.00 & 2 & 0 & 7 & 0 & 91 & 0.077 \\
\hline 77 & 5.28 & 0.90 & 6.88 & 2 & 0 & 8 & 10 & 80 & 0.100 \\
\hline 78 & 25.31 & 2.11 & 5.25 & 0 & 0 & 1 & 1 & 98 & 0.010 \\
\hline 79 & 3.71 & 2.86 & 2.13 & 0 & 0 & 5 & 3 & 92 & 0.054 \\
\hline 80 & 14.02 & 3.49 & 2.13 & 1 & 0 & 1 & 1 & 97 & 0.010 \\
\hline Mea & 8.37 & 3.24 & 6.36 & 1.1 & 0.0 & 4.3 & 2.9 & 91.8 & 0.049 \\
\hline S.E.I & 2.72 & 0.45 & 1.30 & 0.4 & 0.0 & 1.3 & 1.1 & 2.3 & 0.016 \\
\hline
\end{tabular}

\begin{tabular}{|c|c|c|c|c|c|c|c|c|c|}
\hline $\begin{array}{l}\text { Vivariu } \\
\text { Mouse } \\
\text { id \# }\end{array}$ & $\begin{array}{c}\text { um Control Enc } \\
\text { Plasma } \\
\text { Corticosterone } \\
\mu \mathrm{g} / \mathrm{dl}\end{array}$ & $\begin{array}{c}\text { osure, } 8 \\
\text { Plasma } \\
\text { Protein } \\
\text { g/dl }\end{array}$ & $\begin{array}{l}\text { Total } \\
\text { WBC } \\
\text { cells } \times 10^{3} / \mathrm{ul}\end{array}$ & $\begin{array}{c}\text { EOS } \\
\text { count } \\
\% \text { WBC }\end{array}$ & $\begin{array}{c}\text { BAS } \\
\text { count } \\
\% \text { WBC }\end{array}$ & $\begin{array}{c}\text { NEU } \\
\text { count } \\
\% \text { WBC }\end{array}$ & $\begin{array}{c}\text { MON } \\
\text { count } \\
\% \text { WBC } \\
\end{array}$ & $\begin{array}{c}\text { LYM } \\
\text { count } \\
\% \text { WBC } \\
\end{array}$ & $\begin{array}{c}N / L \\
\text { count }\end{array}$ \\
\hline$\overline{81}$ & 17.86 & 1.82 & 4.75 & 3 & 0 & 4 & 0 & 93 & 0.043 \\
\hline 82 & 17.55 & 3.78 & 2.00 & 1 & 0 & 4 & 2 & 93 & 0.043 \\
\hline 83 & 12.12 & 3.65 & 2.63 & 0 & 0 & 3 & 3 & 94 & 0.032 \\
\hline 84 & 12.66 & 4.34 & 4.88 & 3 & 0 & 5 & 5 & 87 & 0.057 \\
\hline 85 & 11.16 & 3.69 & 3.88 & 2 & 0 & 3 & 5 & 90 & 0.033 \\
\hline 86 & 10.22 & 1.14 & 5.50 & 2 & 0 & 4 & 3 & 91 & 0.044 \\
\hline 87 & 10.43 & 1.82 & & & & & & & \\
\hline 88 & & 3.78 & 5.00 & & & & & & \\
\hline$\overline{\text { Mean }}$ & 13.14 & 3.00 & 4.09 & 1.8 & 0.0 & 3.8 & 3.0 & 91.3 & 0.042 \\
\hline S.E.M & 1.22 & 0.43 & 0.50 & 0.5 & 0.0 & 0.3 & 0.8 & 1.1 & 0.004 \\
\hline
\end{tabular}


Table 19 (Experiment 1). Mean \pm S.E.M. white blood cell count (WBC), plasma corticosterone, plasma protein, eosinophil (EOS) count, basophil (BAS) count, neutrophil (NEU) count, monocyte (MON) count, lymphocyte (LYM) count, and neutrophil to lymphocyte ratio (N/L).

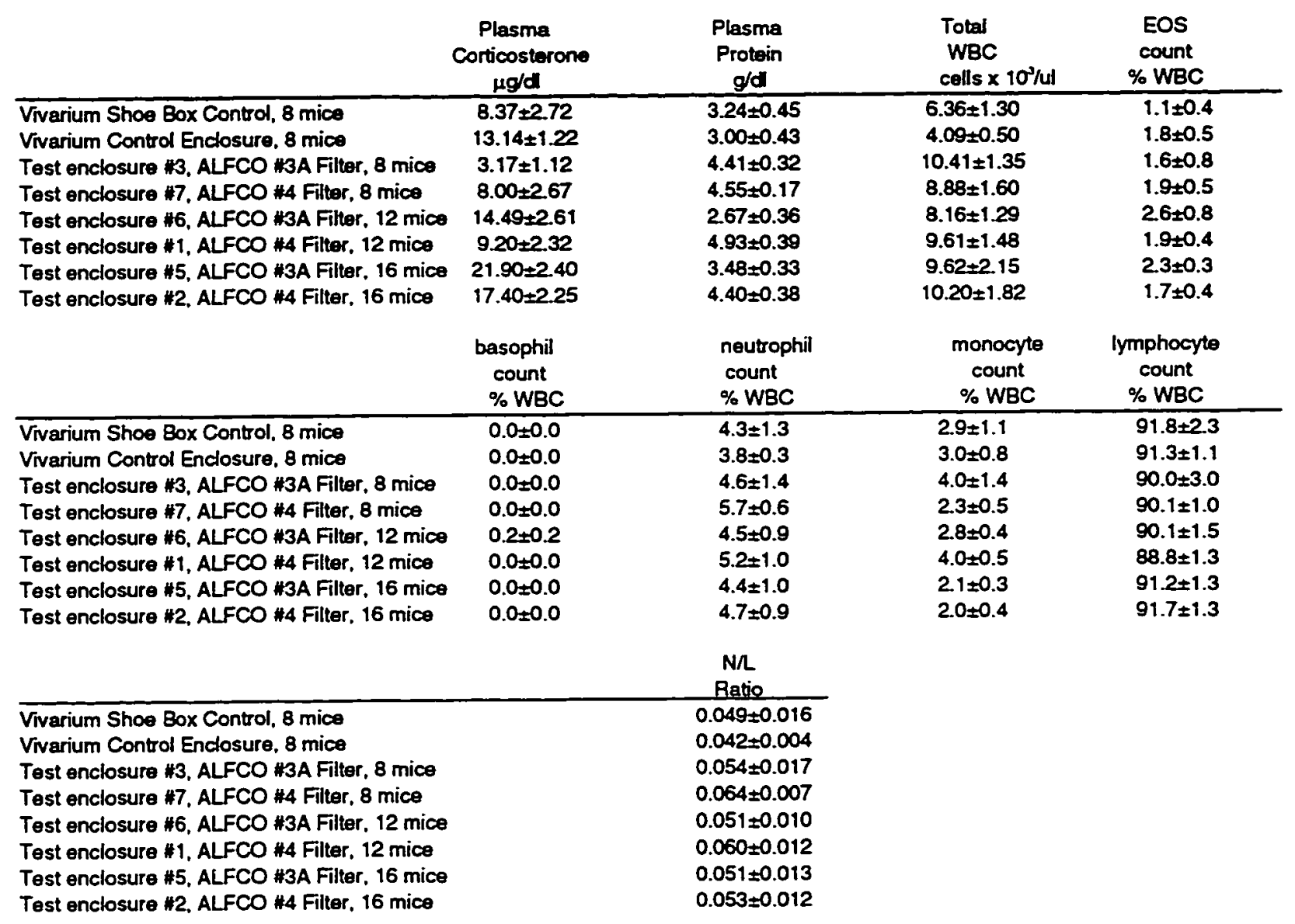


Figure 20 (Experiment 1). Mean \pm S.E.M. plasma corticosterone. and mean \pm S.E.M. plasma protein. (See also Table 19).
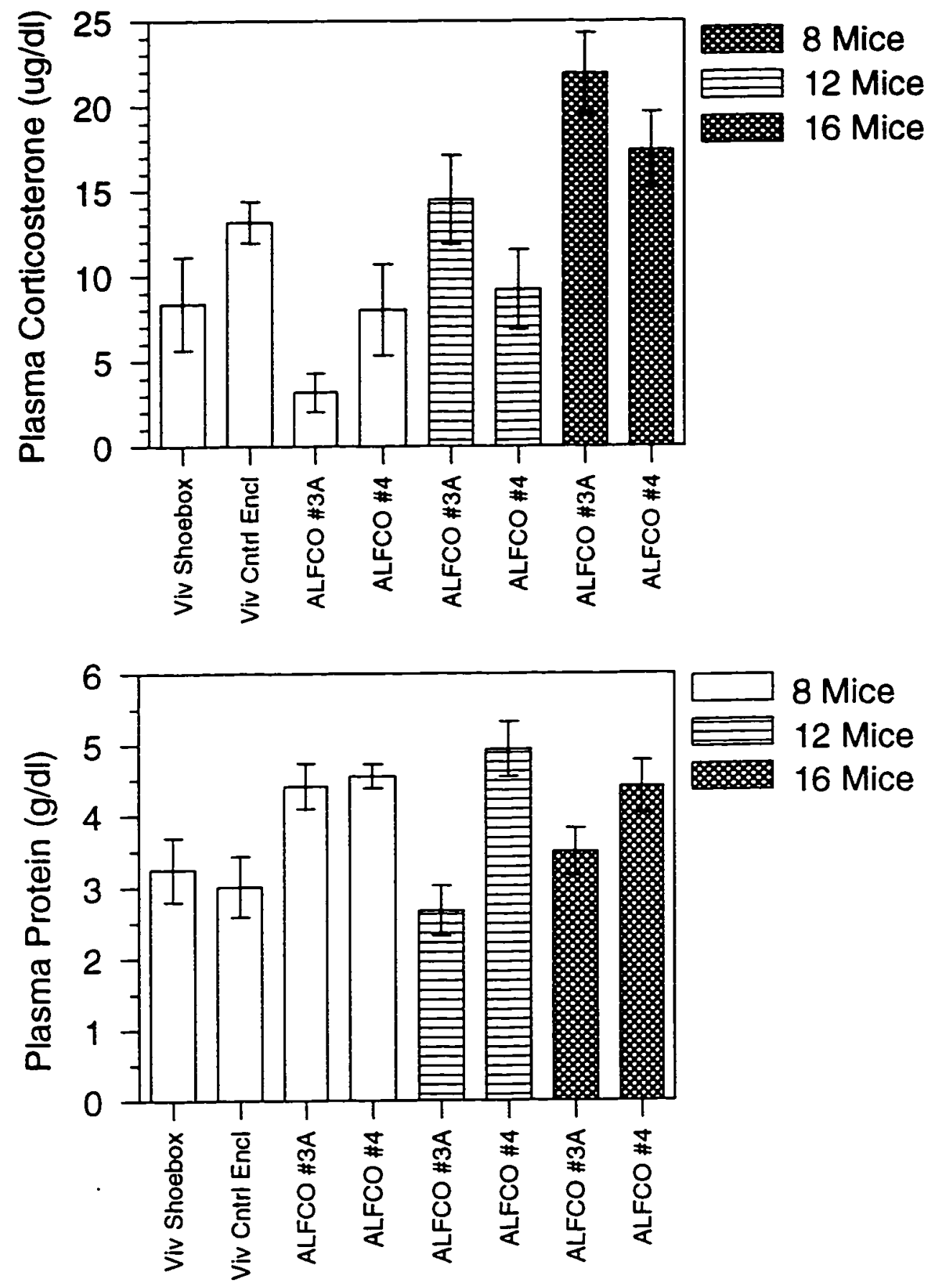
Figure 21 (Experiment 1). Mean \pm S.E.M. total leukocyte count and mean \pm S.E.M. eosinophil count. (See also Table 19).
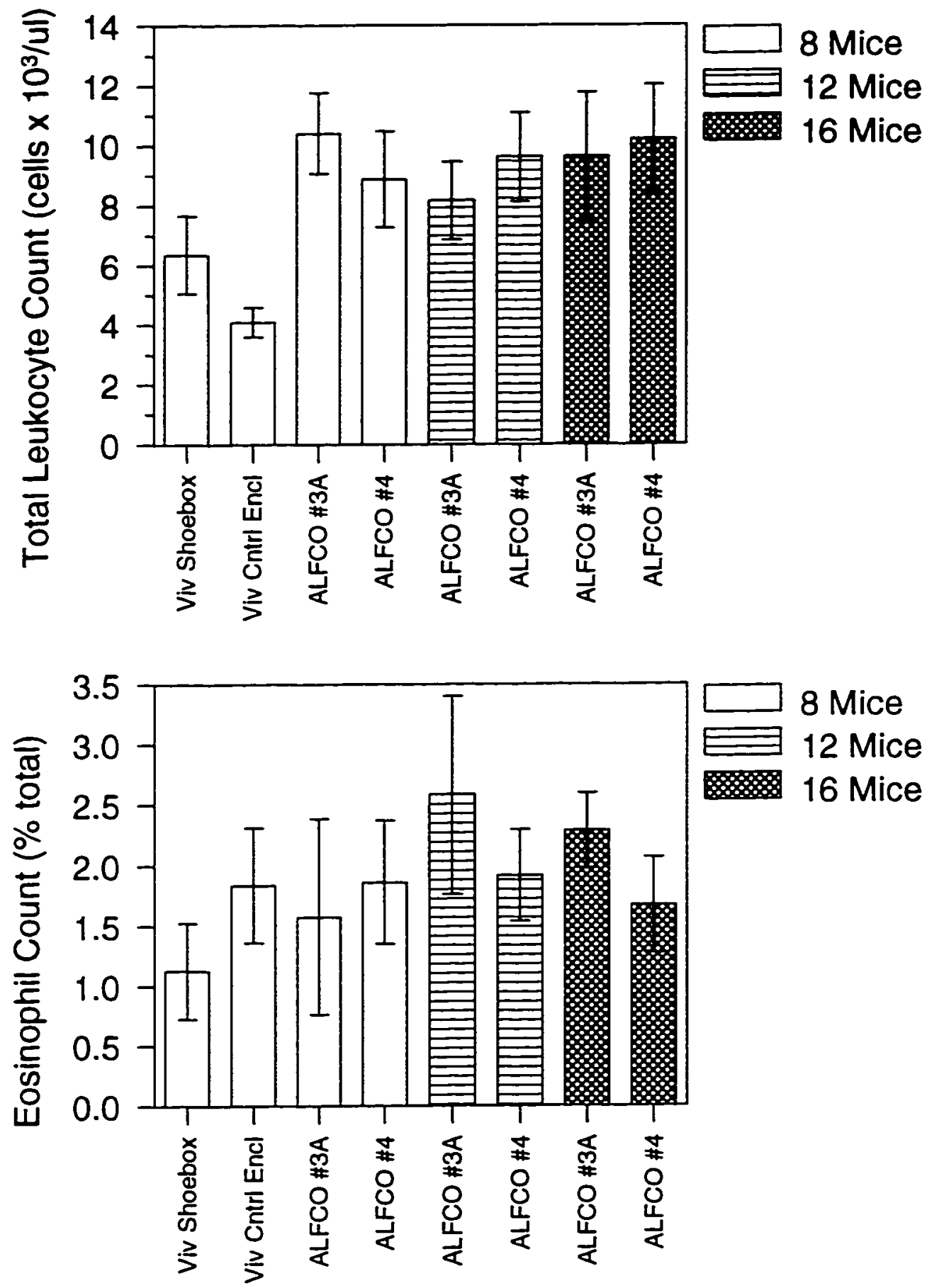
Figure 22 (Experiment 1). Mean \pm S.E.M. basophil count and mean \pm S.E.M. neutrophil count. (See also Table 19).
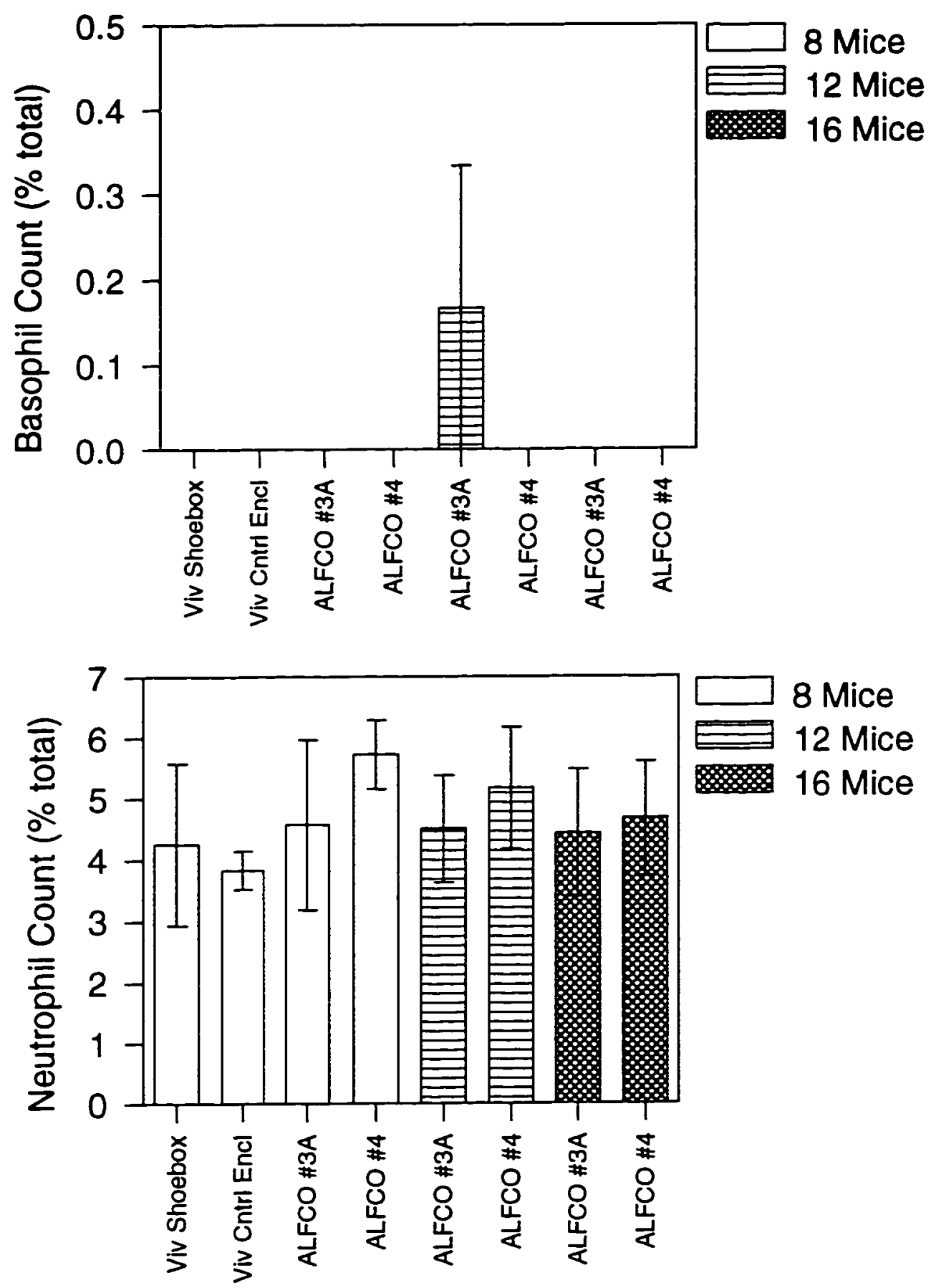
Figure 23 (Experiment 1). Mean \pm S.E.M. monocyte count and mean \pm S.E.M. lymphocyte count. (See also Table 19).
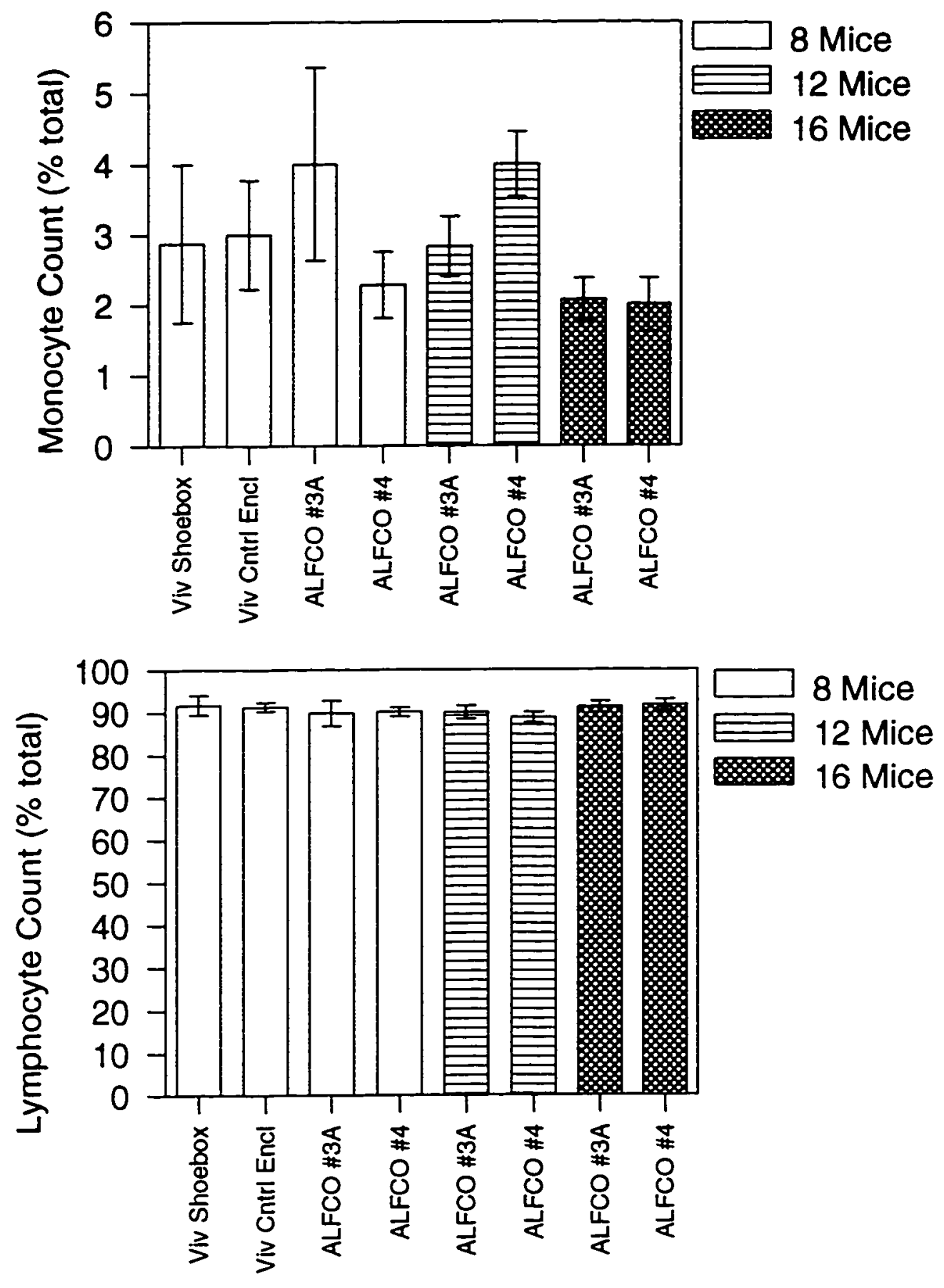
Figure 24 (Experiment 1). Mean \pm S.E.M. neutrophil to lymphocyte ratio. (See also Table 19).

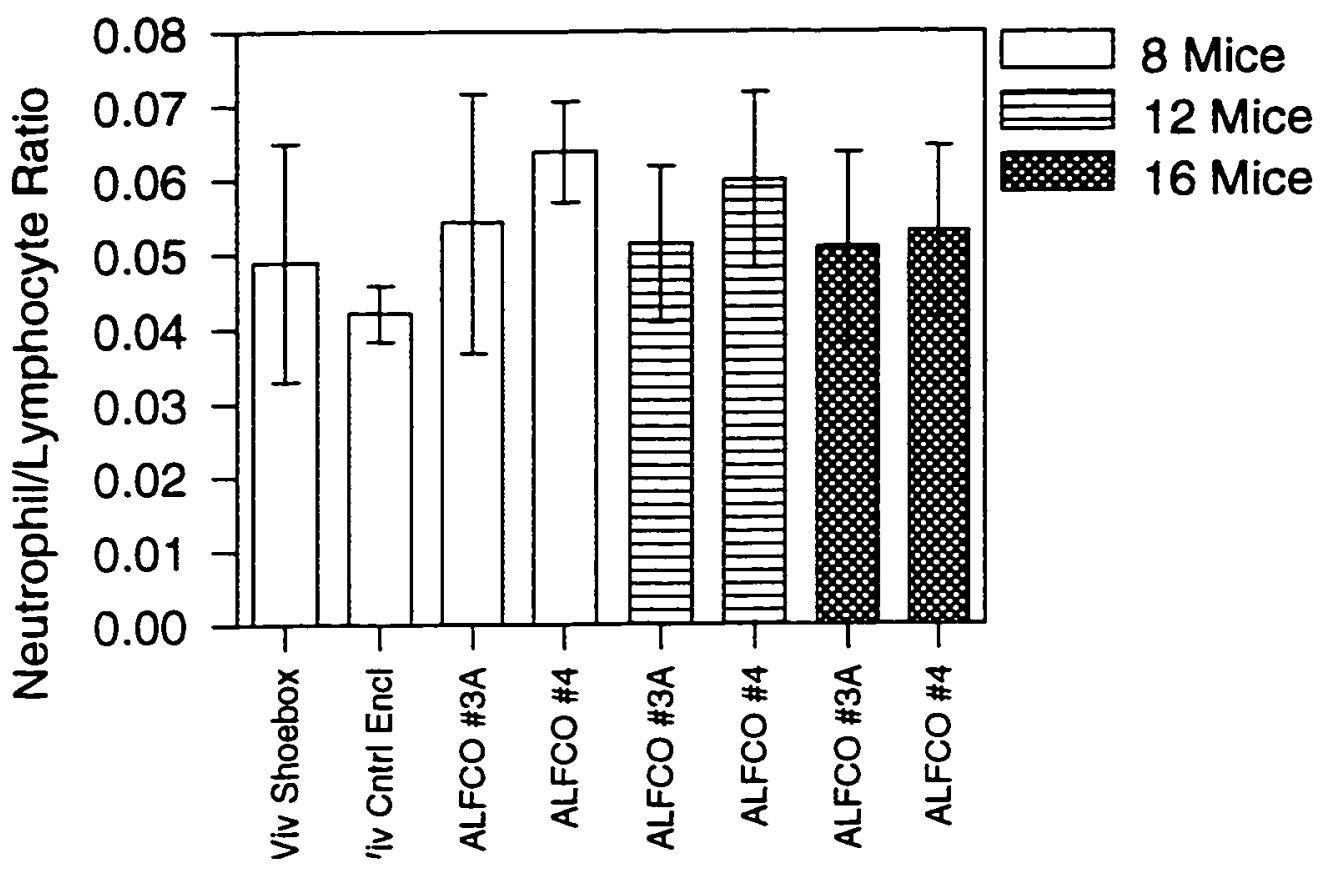


Table 20 (Experiment 1). Video scoring data used for behavioral evaluation. The observer scored ten minutes of video per hour for four hours. Scores represent the total number of times any mouse engaged in the particular activity summed over all quadrants.

Behavioral Score Category

\begin{tabular}{lcccccc}
\hline Number of mice & $\mathbf{0}$ & $\mathbf{1}$ & $\mathbf{2}$ & $\mathbf{3}$ & $\mathbf{4}$ & $\mathbf{5}$ \\
\hline Test Enclosure \#6, ALFCO \#3A, 12 mice & 198 & 7 & 544 & 0 & 0 & 9 \\
Test Enclosure \#5, ALFCO \#3A, 16 mice & 229 & 39 & 929 & 0 & 0 & 20 \\
Test Enclosure \#1, ALFCO \#4, 12 mice & 200 & 37 & 567 & 16 & 0 & 30 \\
Test Enclosure \#2, ALFCO \#4, 16 mice & 157 & 49 & 701 & 15 & 0 & 79
\end{tabular}

Behavioral Score Category Definitions

0 - sleeping, still, sniffing

1 - eating, drinking

2 - grooming, moving

3 - social interaction

4 - aggressive behavior

5 - running 
Table 21 (Experiment 1). Cage temperatures $\left({ }^{\circ} \mathrm{C}\right)$ for each test enclosure and the daily vivarium room temperature at the time of animal maintenance.

\begin{tabular}{|c|c|c|c|c|c|c|c|}
\hline $\begin{array}{c}\text { Day } \\
\text { Number }\end{array}$ & $\begin{array}{c}\text { Test Encl } \\
\# 3 \\
\text { (8 mice) }\end{array}$ & $\begin{array}{c}\text { Test Encl } \\
\# 6 \\
\text { (12 mice) }\end{array}$ & $\begin{array}{c}\text { Test Encl } \\
\# 5 \\
(16 \text { mice) }\end{array}$ & $\begin{array}{c}\text { Test Encl } \\
\# 7 \\
(8 \text { mice) }\end{array}$ & $\begin{array}{c}\text { Test Encl } \\
\# 1 \\
\text { (12 mice) }\end{array}$ & $\begin{array}{c}\text { Test Encl } \\
\# 2 \\
(16 \text { mice) }\end{array}$ & $\begin{array}{l}\text { Vivarium } \\
\text { (8 mice) }\end{array}$ \\
\hline 1 & 22.5 & 23.0 & 23.0 & 23.0 & 23.0 & 23.5 & \\
\hline 2 & 22.0 & 23.5 & 23.0 & 24.0 & 23.0 & 23.0 & \\
\hline 3 & 24.0 & 24.5 & 24.5 & 24.5 & 24.5 & 24.5 & 21.0 \\
\hline 4 & 24.5 & 24.0 & 25.0 & 25.0 & 26.0 & 25.5 & 19.0 \\
\hline 5 & & & & & & & \\
\hline 6 & 26.0 & 26.0 & 26.0 & 26.0 & 26.0 & 26.0 & \\
\hline 7 & 24.0 & 23.5 & 23.5 & 24.0 & 23.5 & 24.0 & \\
\hline 8 & 24.0 & 24.0 & 24.0 & 24.0 & 24.0 & 25.0 & \\
\hline 9 & 24.5 & 24.0 & 25.0 & 24.5 & 24.5 & 25.0 & 24.5 \\
\hline 10 & 25.0 & 24.5 & 25.0 & 25.0 & 24.5 & 25.0 & 24.5 \\
\hline 11 & 24.5 & 25.0 & 25.0 & 25.0 & 24.5 & 25.0 & 24.5 \\
\hline 12 & 25.0 & 25.0 & 24.5 & 25.0 & 24.5 & 25.0 & 24.5 \\
\hline 13 & 23.0 & 23.0 & 23.0 & 23.0 & 23.0 & 24.0 & 24.5 \\
\hline 14 & 23.0 & 23.0 & 23.0 & 23.0 & 23.0 & 23.5 & 25.0 \\
\hline 15 & 24.0 & 24.0 & 24.0 & 24.0 & 24.0 & 24.0 & 24.0 \\
\hline 16 & 25.0 & 25.0 & 25.0 & 25.0 & 24.0 & 25.0 & 24.0 \\
\hline 17 & & 24.0 & 24.0 & 24.0 & 23.5 & 24.0 & 24.0 \\
\hline 18 & 24.0 & 24.0 & 24.0 & 24.0 & 24.0 & 24.0 & 24.5 \\
\hline 19 & 21.5 & 22.0 & 22.0 & 22.0 & 22.0 & 22.0 & 24.5 \\
\hline 20 & 22.0 & 22.0 & 22.0 & 22.0 & 22.0 & 22.0 & 24.5 \\
\hline 21 & & 21.5 & 21.5 & 21.5 & 21.5 & 21.5 & 23.5 \\
\hline 22 & & 23.0 & 23.0 & 23.0 & 23.0 & 23.0 & 23.5 \\
\hline 23 & 24.5 & 24.0 & 24.0 & 24.0 & 24.0 & 24.0 & 25.5 \\
\hline 24 & 24.0 & 24.0 & 24.0 & 24.0 & 23.5 & 24.0 & 24.0 \\
\hline 25 & 23.5 & 23.5 & 23.5 & 24.0 & 23.5 & 24.0 & 24.5 \\
\hline 26 & 25.0 & 25.0 & 25.0 & 25.0 & 25.0 & 25.0 & 22.0 \\
\hline 27 & 23.0 & 23.0 & 23.0 & 23.0 & 23.0 & 23.0 & 20.0 \\
\hline 28 & 22.0 & 22.0 & 22.0 & 22.0 & 22.0 & 22.0 & 16.0 \\
\hline 29 & 25.0 & 25.0 & 25.0 & 25.0 & 25.0 & 25.0 & 30.0 \\
\hline 30 & & 24.0 & 26.0 & 26.0 & 25.0 & 25.0 & \\
\hline
\end{tabular}


Table 22 (Experiment 1). Results of testing for the effects of the number of mice per cage and the filter type used. Column two shows adjusted $G$ from the test for normally distributed data. A single asterisk indicates data that is not normally distributed $\left(G_{a d j}>\chi^{2}{ }_{.05}(\eta)\right.$. Column five shows Burr-Foster q-test results for homogeneity of variances. Two asterisks indicates data where the variances are

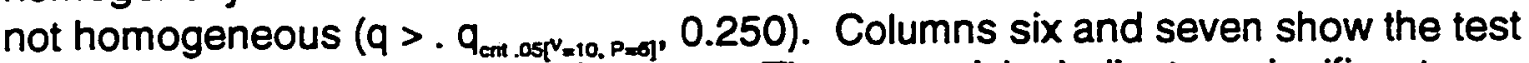
used and significance results of the test. Three asterisks indicate a significant difference found.

\begin{tabular}{|c|c|c|c|c|c|c|}
\hline & $G_{\text {adj }}$ & $\underline{v}$ & $x^{2}-0.05[v]$ & $q$ & Test Used & Significanc \\
\hline Initial Wt (grams) & 15.795 & 10 & 18.307 & 0.175 & ANOVA & \\
\hline Final Wt (grams) & 5.421 & 11 & 19.675 & 0.196 & ANOVA & \\
\hline Gain (grams) & 17.513 & 12 & 21.026 & 0.192 & ANOVA & \\
\hline $\arcsin ($ sqrt(Gain (\%body)))) & 8.712 & 11 & 19.675 & 0.188 & ANOVA & \\
\hline Heart Wt (grams) & 5.814 & 10 & 18.307 & 0.194 & ANOVA & \\
\hline $\arcsin ($ sqrt(Heart Wt (\%body))) & 11.210 & 10 & 18.307 & 0.175 & ANOVA & \\
\hline Kidney Wt (grams) *" & 11.165 & 9 & 16.919 & 0.253 & N/A & \\
\hline Sqrt (Kidney Wt) & 12.376 & 11 & 19.675 & 0.180 & ANOVA & \\
\hline $\arcsin ($ sqrt(Kidney Wt (\%body))) & 3.416 & 12 & 21.026 & 0.189 & ANOVA & $* * \#$ \\
\hline Adrenal Wt (grams) & 10.250 & 11 & 19.675 & 0.194 & ANOVA & \\
\hline $\arcsin ($ sqrt(Adrenal Wt (\%body))) & 14.867 & 12 & 21.026 & 0.192 & ANOVA & \\
\hline Spleen Wt (grams) & 21.792 & 11 & 19.675 & 0.185 & Kruskal-Wallis & \\
\hline Sqrt (Spleen Wt) : & 23.901 & 12 & 21.026 & 0.139 & N/A & \\
\hline $\arcsin ($ sqrt(Spleen Wt (\%body))) & 10.508 & 10 & 18.307 & 0.221 & ANOVA & \\
\hline Thymus Wt (grams) & 10.537 & 15 & 24.996 & 0.201 & ANOVA & $* * F$ \\
\hline $\arcsin ($ sqrt(Thymus Wt (\%body))) & 10.871 & 12 & 21.026 & 0.208 & ANOVA & $\cdots F$ \\
\hline Plasma Corticosterone & 32.661 & 11 & 19.675 & 0.209 & N/A & \\
\hline sqrt(Plasma Corticostero & 7.100 & 12 & 21.026 & 0.191 & ANOVA & $\cdots \# F$ \\
\hline Plasma Protein & 8.714 & 11 & 19.675 & 0.239 & ANOVA & $\cdots \#$ \\
\hline Total Leukocyte & 30.974 & 13 & 22.362 & 0.226 & N/A & \\
\hline squt(Total Leukocyte) & 10.958 & 10 & 18.307 & 0.199 & ANOVA & \\
\hline
\end{tabular}

\# - Significant result associated with number of mice per cage.

F - Significant result associated with filter type. 
Table 23 (Experiment 1). Results of testing for the effects of the cage type used. Column two shows adjusted $G$ from the test for normally distributed data. A single asterisk indicates data that is not normally distributed $\left(G_{a d j}>\chi^{2}{ }_{.05}(y)\right.$. Column five shows Burr-Foster $q$-test results for homogeneity of variances. Two asterisks

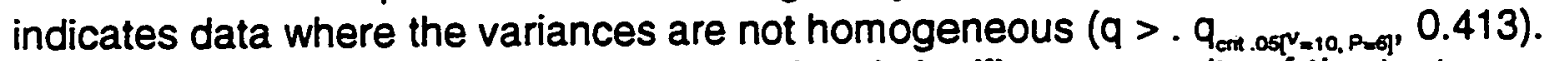
Columns six and seven show the test used and significance results of the test. Three asterisks indicate a significant difference found.

\begin{tabular}{|c|c|c|c|c|c|c|}
\hline & $\underline{G}_{\text {adj- }}$ & $\underline{v}$ & $x^{2} \underline{0.05[v]}$ & $q$ & Test Used & Results \\
\hline Initial Wt (grams) & 3.211 & 7 & 14.067 & 0.260 & ANOVA & \\
\hline Final Wt (grams) & 7.382 & 10 & 18.307 & 0.328 & ANOVA & \\
\hline Gain (grams) & 9.844 & 6 & 12.592 & 0.367 & ANOVA & \\
\hline $\arcsin (\operatorname{sqr}($ Gain $(\%$ body $))))^{*}$ & 20.025 & 9 & 16.919 & 0.347 & Kruskal Wallis & \\
\hline Heart Wt (grams) & 7.603 & 10 & 18.307 & 0.294 & ANOVA & $* *$ \\
\hline $\arcsin (\operatorname{sgrt}($ Heart Wt (\%body))) & 5.031 & 7 & 14.067 & 0.260 & ANOVA & $\cdots$ \\
\hline Kidney Wt (grams) & 8.680 & 9 & 16.919 & 0.310 & ANOVA & \\
\hline $\arcsin ($ sqrt(Kidney Wt (\%body))) & 6.742 & 9 & 16.919 & 0.330 & ANOVA & \\
\hline Adrenal Wt (grams) & 3.315 & 7 & 14.067 & 0.346 & ANOVA & \\
\hline $\arcsin ($ sqrt(Adrenal Wt (\%body))) & 8.968 & 10 & 18.307 & 0.366 & ANOVA & \\
\hline Spleen Wt (grams) & 18.055 & 12 & 21.026 & 0.274 & ANOVA & \\
\hline $\arcsin ($ sqrt(Spleen Wt (\%body))) & 5.989 & 10 & 18.307 & 0.285 & ANOVA & \\
\hline Thymus Wt (grams) & 7.204 & 11 & 19.675 & 0.319 & ANOVA & \\
\hline $\arcsin ($ sqrt(Thymus Wt (\%body))) & 9.548 & 10 & 18.307 & 0.346 & ANOVA & $\cdots$ \\
\hline Plasma Corticosterone & 21.349 & 10 & 18.307 & 0.398 & N/A & \\
\hline sqrt(Plasma Corticosterone) & 11.692 & 9 & 16.919 & 0.342 & ANOVA & $*$ \\
\hline Plasma Protein & 6.742 & 9 & 16.919 & 0.327 & ANOVA & *** \\
\hline Total Leukocyte & 12.535 & 12 & 21.026 & 0.345 & ANOVA & *** \\
\hline
\end{tabular}




\section{Experiment 2: Mouse Density Study}

Table 24 (Experiment 2). Daily total and per mouse water consumption for each cage $(\mathrm{ml})$. Mean \pm S.E.M. were calculated using test days 4 through 30 only (See also Figure 25).

\begin{tabular}{|c|c|c|c|c|c|c|c|c|c|c|}
\hline \multirow[t]{2}{*}{$\begin{array}{l}\text { Test Day } \\
\text { Number }\end{array}$} & \multicolumn{2}{|c|}{$\frac{\text { Test Encl \#2 }}{(8 \text { mice) }}$} & \multicolumn{2}{|c|}{$\frac{\text { Test Encl \#4 }}{(12 \text { mice })}$} & \multicolumn{2}{|c|}{$\frac{\text { Test Encl \#1 }}{(16 \text { mice })}$} & \multicolumn{2}{|c|}{$\frac{\text { VivShoeBox }}{(8 \text { mice })}$} & \multicolumn{2}{|c|}{$\frac{\text { VivCntrlEncl }}{(8 \text { mice) }}$} \\
\hline & $\mathrm{ml}$ & $\begin{array}{l}\text { ml per } \\
\text { mouse }\end{array}$ & $\mathrm{ml}$ & $\begin{array}{l}\text { ml per } \\
\text { mouse }\end{array}$ & $\mathrm{ml}$ & $\begin{array}{l}\mathrm{ml} \text { per } \\
\text { mouse }\end{array}$ & $\mathrm{ml}$ & $\begin{array}{l}\text { ml per } \\
\text { mouse }\end{array}$ & $\mathrm{ml}$ & $\begin{array}{l}\text { ml per } \\
\text { mouse }\end{array}$ \\
\hline 1 & 28 & 3.5 & 40 & 3.3 & 48 & 3.0 & 50 & 6.3 & 48 & 6.0 \\
\hline 2 & 40 & 5.0 & 52 & 4.3 & 67 & 4.2 & 52 & 6.5 & 64 & 8.0 \\
\hline 3 & 60 & 7.5 & 74 & 6.2 & 90 & 5.6 & 45 & 5.6 & 40 & 5.0 \\
\hline 4 & 52 & 6.5 & 70 & 5.8 & 85 & 5.3 & 41 & 5.1 & 50 & 6.3 \\
\hline 5 & 45 & 5.6 & 80 & 6.7 & 78 & 4.9 & 21 & 2.6 & 19 & 2.4 \\
\hline 6 & 50 & 6.3 & 88 & 7.3 & 90 & 5.6 & 47 & 5.9 & 56 & 7.0 \\
\hline 7 & 50 & 6.3 & 56 & 4.7 & 93 & 5.8 & 60 & 7.5 & 94 & 11.8 \\
\hline 8 & 56 & 7.0 & 72 & 6.0 & 102 & 6.4 & 58 & 7.3 & 52 & 6.5 \\
\hline 9 & 76 & 9.5 & 81 & 6.8 & 108 & 6.8 & 70 & 8.8 & 35 & 4.4 \\
\hline 10 & 63 & 7.9 & 84 & 7.0 & 109 & 6.8 & 42 & 5.3 & 30 & 3.8 \\
\hline 11 & 62 & 7.8 & 80 & 6.7 & 102 & 6.4 & 54 & 6.8 & 52 & 6.5 \\
\hline 12 & 62 & 7.8 & 76 & 6.3 & 108 & 6.8 & n.d. & n.d. & n.d. & n.d. \\
\hline 13 & 78 & 9.8 & 100 & 8.3 & 150 & 9.4 & 52 & 6.5 & 54 & 6.8 \\
\hline 14 & 65 & 8.1 & 73 & 6.1 & 148 & 9.3 & 50 & 6.3 & 58 & 7.3 \\
\hline 15 & 69 & 8.6 & 95 & 7.9 & 100 & 6.3 & 45 & 5.6 & n.d. & n.d. \\
\hline 16 & 65 & 8.1 & 116 & 9.7 & 125 & 7.8 & 58 & 7.3 & 55 & 6.9 \\
\hline 17 & 75 & 9.4 & 119 & 9.9 & 123 & 7.7 & 51 & 6.4 & 60 & 7.5 \\
\hline 18 & 57 & 7.1 & 92 & 7.7 & 140 & 8.8 & 52 & 6.5 & 61 & 7.6 \\
\hline 19 & 85 & 10.6 & 112 & 9.3 & 158 & 9.9 & 56 & 7.0 & 72 & 9.0 \\
\hline 20 & 82 & 10.3 & 115 & 9.6 & 156 & 9.8 & 63 & 7.9 & 70 & 8.8 \\
\hline 21 & 80 & 10.0 & 98 & 8.2 & 132 & 8.3 & 72 & 9.0 & 76 & 9.5 \\
\hline 22 & 56 & 7.0 & 90 & 7.5 & 141 & 8.8 & 54 & 6.8 & 60 & 7.5 \\
\hline 23 & 56 & 7.0 & 70 & 5.8 & 97 & 6.1 & 67 & 8.4 & 64 & 8.0 \\
\hline 24 & 79 & 9.9 & 83 & 6.9 & 90 & 5.6 & 61 & 7.6 & 72 & 9.0 \\
\hline 25 & 72 & 9.0 & 78 & 6.5 & 95 & 5.9 & 60 & 7.5 & 68 & 8.5 \\
\hline 26 & 87 & 10.9 & 100 & 8.3 & 108 & 6.8 & 50 & 6.3 & 56 & 7.0 \\
\hline 27 & 86 & 10.8 & 94 & 7.8 & 126 & 7.9 & 58 & 7.3 & 63 & 7.9 \\
\hline 28 & 80 & 10.0 & 92 & 7.7 & 125 & 7.8 & 70 & 8.8 & 78 & 9.8 \\
\hline 29 & 79 & 9.9 & 85 & 7.1 & 115 & 7.2 & 64 & 8.0 & 44 & 5.5 \\
\hline 30 & 41 & 5.1 & 82 & 6.8 & 82 & 5.1 & n.d. & n.d. & & n.d. \\
\hline Mean & & 8.4 & & 7.4 & & 7.1 & & 6.9 & & 7.3 \\
\hline SEM & & 0.32 & & 0.25 & & 0.28 & & 0.28 & & 0.41 \\
\hline
\end{tabular}


Figure 25 (Experiment 2): Daily water consumption per mouse for each cage.

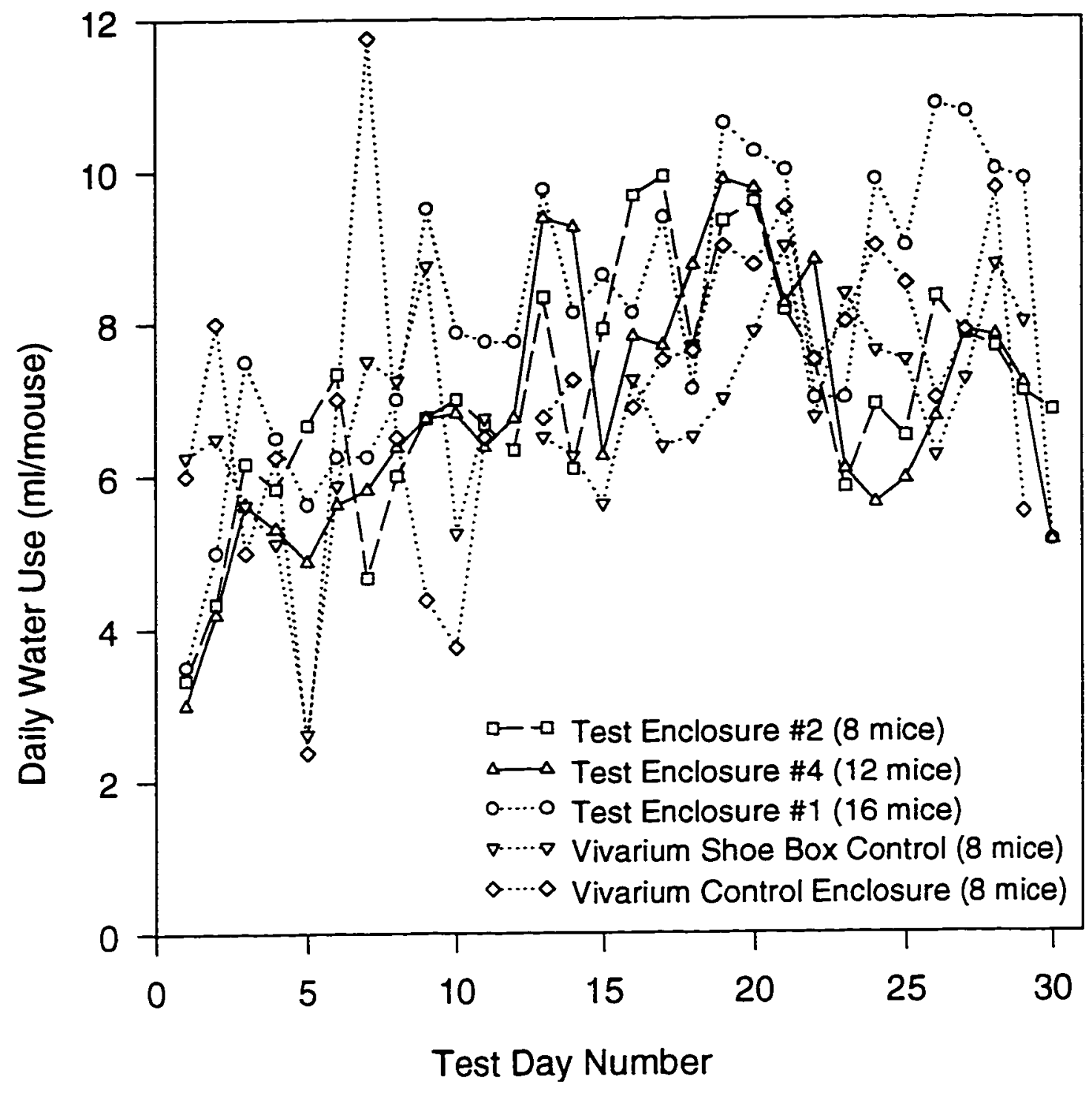


Table 25 (Experiment 2). Food consumption (grams) over 30 days.

\begin{tabular}{lccccc} 
& $\begin{array}{c}\text { Test Enclosure } \\
\# 2,8 \text { mice }\end{array}$ & $\begin{array}{c}\text { Test Enclosure } \\
\# 4,12 \text { mice }\end{array}$ & $\begin{array}{c}\text { Test Enclosure } \\
\# 1,16 \text { mice }\end{array}$ & $\begin{array}{c}\text { Vivarium Shoe } \\
\text { Box Control, 8 }\end{array}$ & $\begin{array}{c}\text { Vivarium Cntrl } \\
\text { Enclosure, 8 }\end{array}$ \\
\hline Total & 1234.3 & 1660.6 & 1461.5 & 977.0 & 1212.9 \\
TotaVmouse/day & 154.3 & 138.4 & 91.3 & 122.1 & 151.6
\end{tabular}

Table 26 (Experiment 2). Mouse initial (test day 0 ) and final (test day 30 ) body weight, weight gain in grams, and $\%$ weight gain.

\begin{tabular}{lccccc} 
& $\begin{array}{c}\text { Mouse } \\
\#\end{array}$ & $\begin{array}{c}\text { Initial } \\
\text { Wt }(\mathrm{g})\end{array}$ & $\begin{array}{c}\text { Final } \\
\text { Wt }(\mathrm{g})\end{array}$ & $\begin{array}{c}\text { Gain } \\
(\mathrm{g})\end{array}$ & $\begin{array}{c}\text { Gain } \\
\text { (\% Initial Weight) }\end{array}$ \\
\hline Test Enclosure \#2 & 1 & 14.91 & 18.19 & 3.28 & 22.00 \\
ALFCO \#3A Filter & 2 & 18.72 & 26.19 & 7.47 & 39.90 \\
8 mice & 3 & 17.35 & 29.53 & 12.18 & 70.20 \\
& 4 & 16.80 & 26.94 & 10.14 & 60.36 \\
& 5 & 18.98 & 26.83 & 7.85 & 41.36 \\
& 6 & 16.11 & 27.60 & 11.49 & 71.32 \\
& 7 & 16.50 & 33.15 & 16.65 & 100.91 \\
\hline Mean & 8 & 18.65 & 30.38 & 11.73 & 62.90 \\
S.E.M. & & 17.25 & 27.35 & 10.10 & 58.62 \\
& & 0.51 & 1.54 & 1.40 & 8.55 \\
\hline & & & & & \\
& & & & & \\
& Mouse & 1 Initial & Final & Gain & Gain \\
\hline Test Enclosure \#4 & $\#$ & Wt (g) & Wt (g) & $(\mathrm{g})$ & (\% Initial Weight) \\
ALFCO \#3A Filter & 9 & 17.20 & 27.73 & 10.53 & 61.22 \\
12 mice & 10 & 16.33 & 26.84 & 10.51 & 64.36 \\
& 11 & 18.10 & 27.97 & 9.87 & 54.53 \\
& 12 & 17.58 & 30.49 & 12.91 & 73.44 \\
& 13 & 18.56 & 31.32 & 12.76 & 68.75 \\
& 14 & 18.12 & 26.98 & 8.86 & 48.90 \\
& 15 & 17.35 & 30.16 & 12.81 & 73.83 \\
& 16 & 17.13 & 31.99 & 14.86 & 86.75 \\
& 17 & 17.97 & 26.83 & 8.86 & 49.30 \\
Sean & 18 & 17.91 & 30.81 & 12.90 & 72.03 \\
& 19 & 15.82 & 26.93 & 11.11 & 70.23 \\
& 20 & 17.62 & 27.66 & 10.04 & 56.98 \\
\hline & & 17.47 & 28.81 & 11.34 & 65.03 \\
& & 0.23 & 0.57 & 0.54 & 3.27 \\
\hline & & & & &
\end{tabular}




\begin{tabular}{|c|c|c|c|c|c|}
\hline & $\begin{array}{l}\text { Mouse } \\
\#\end{array}$ & $\begin{array}{l}\text { Initial } \\
\text { Wt (g) }\end{array}$ & $\begin{array}{l}\text { Final } \\
\text { Wt (g) }\end{array}$ & $\begin{array}{c}\text { Gain } \\
\text { (g) }\end{array}$ & $\begin{array}{l}\text { Gain } \\
\text { (\% Initial Weight) }\end{array}$ \\
\hline $\begin{array}{l}\text { Test Enclosure \#1 } \\
\text { ALFCO \#3A Filter } \\
16 \text { mice }\end{array}$ & $\begin{array}{l}21 \\
22 \\
23 \\
24 \\
25 \\
26 \\
27 \\
28 \\
29 \\
30 \\
31 \\
32 \\
33 \\
34 \\
35 \\
36\end{array}$ & $\begin{array}{l}16.68 \\
16.61 \\
17.82 \\
16.99 \\
17.58 \\
17.08 \\
16.79 \\
18.92 \\
17.19 \\
17.49 \\
16.83 \\
16.70 \\
18.35 \\
15.22 \\
16.68 \\
17.72\end{array}$ & $\begin{array}{l}25.67 \\
29.00 \\
28.37 \\
28.85 \\
27.52 \\
26.78 \\
31.00 \\
25.50 \\
28.36 \\
26.11 \\
28.52 \\
29.48 \\
30.99 \\
30.49 \\
28.93 \\
28.18\end{array}$ & $\begin{array}{r}8.99 \\
12.39 \\
10.55 \\
11.86 \\
9.94 \\
9.70 \\
14.21 \\
6.58 \\
11.17 \\
8.62 \\
11.69 \\
12.78 \\
12.64 \\
15.27 \\
12.25 \\
10.46\end{array}$ & $\begin{array}{r}53.90 \\
74.59 \\
59.20 \\
69.81 \\
56.54 \\
56.79 \\
84.63 \\
34.78 \\
64.98 \\
49.29 \\
69.46 \\
76.53 \\
68.88 \\
100.33 \\
73.44 \\
59.03\end{array}$ \\
\hline \multirow[t]{2}{*}{$\begin{array}{l}\text { Mean } \\
\text { S.E.M. }\end{array}$} & & $\begin{array}{r}17.17 \\
0.21 \\
\end{array}$ & $\begin{array}{r}28.36 \\
0.43 \\
\end{array}$ & $\begin{array}{r}11.19 \\
0.54 \\
\end{array}$ & $\begin{array}{r}65.76 \\
3.80 \\
\end{array}$ \\
\hline & $\begin{array}{c}\text { Mouse } \\
\#\end{array}$ & $\begin{array}{l}\text { Initial } \\
\text { Wt (g) }\end{array}$ & $\begin{array}{l}\text { Final } \\
\text { Wt (g) }\end{array}$ & $\begin{array}{l}\text { Gain } \\
(\mathrm{g})\end{array}$ & $\begin{array}{c}\text { Gain } \\
\text { (\% Initial Weight) }\end{array}$ \\
\hline $\begin{array}{l}\text { Vivarium Shoe Box Control } \\
8 \text { mice }\end{array}$ & $\begin{array}{l}37 \\
38 \\
39 \\
40 \\
41 \\
42 \\
43 \\
44\end{array}$ & $\begin{array}{l}17.70 \\
16.94 \\
17.02 \\
16.89 \\
17.73 \\
18.19 \\
16.62 \\
17.71 \\
\end{array}$ & $\begin{array}{l}29.93 \\
30.19 \\
29.82 \\
25.92 \\
30.05 \\
28.42 \\
29.35 \\
28.52 \\
\end{array}$ & $\begin{array}{r}12.23 \\
13.25 \\
12.80 \\
9.03 \\
12.32 \\
10.23 \\
12.73 \\
10.81 \\
\end{array}$ & $\begin{array}{l}69.10 \\
78.22 \\
75.21 \\
53.46 \\
69.49 \\
56.24 \\
76.59 \\
61.04 \\
\end{array}$ \\
\hline $\begin{array}{l}\text { Mean } \\
\text { S.E.M. }\end{array}$ & & $\begin{array}{r}17.35 \\
0.19\end{array}$ & $\begin{array}{r}29.03 \\
0.50 \\
\end{array}$ & $\begin{array}{r}11.67 \\
0.52 \\
\end{array}$ & $\begin{array}{r}67.42 \\
3.35 \\
\end{array}$ \\
\hline
\end{tabular}




\begin{tabular}{lccccc} 
& $\begin{array}{c}\text { Mouse } \\
\#\end{array}$ & $\begin{array}{c}\text { Initial } \\
\text { Wt }(\mathrm{g})\end{array}$ & $\begin{array}{c}\text { Final } \\
\text { Wt }(\mathrm{g})\end{array}$ & $\begin{array}{c}\text { Gain } \\
(\mathrm{g})\end{array}$ & $\begin{array}{c}\text { Gain } \\
\text { (\% Initial Weight) }\end{array}$ \\
\hline Vivarium Control Enclosure & 45 & 17.92 & 30.20 & 12.28 & 68.53 \\
8 mice & 46 & 18.32 & 28.96 & 10.64 & 58.08 \\
& 47 & 17.02 & 31.10 & 14.08 & 82.73 \\
& 48 & 19.63 & 32.52 & 12.89 & 65.66 \\
& 49 & 16.62 & 30.16 & 13.54 & 81.47 \\
& 50 & 17.15 & 26.50 & 9.35 & 54.52 \\
& 51 & 16.41 & 23.90 & 7.49 & 45.64 \\
\hline Mean & 52 & 17.40 & 30.62 & 13.22 & 75.98 \\
S.E.M. & & 17.56 & 29.24 & 11.69 & 66.58 \\
\hline
\end{tabular}

Table 27 (Experiment 2). Cage mean \pm S.E.M. for initial (test day 0 ) and final (test day 30 ) body weight, weight gain in grams, and \% weight gain.

\begin{tabular}{lcccc} 
& $\begin{array}{c}\text { Initial } \\
\text { Wt }(\mathrm{g})\end{array}$ & $\begin{array}{c}\text { Final } \\
\text { Wt }(\mathrm{g})\end{array}$ & $\begin{array}{c}\text { Gain } \\
(\mathrm{g})\end{array}$ & $\begin{array}{c}\text { Gain } \\
\text { (\% Initial Weight) }\end{array}$ \\
\hline Test Enclosure \#2, 8 mice & $17.25 \pm 0.51$ & $27.35 \pm 1.54$ & $10.10 \pm 1.40$ & $58.62 \pm 8.55$ \\
Test Enclosure \#4, 12 mice & $17.47 \pm 0.23$ & $28.81 \pm 0.57$ & $11.34 \pm 0.54$ & $65.03 \pm 3.27$ \\
Test Enclosure \#1, 16 mice & $17.17 \pm 0.21$ & $28.36 \pm 0.43$ & $11.19 \pm 0.54$ & $65.76 \pm 3.80$ \\
Vivarium Shoe Box Cntrl, 8 mice & $17.35 \pm 0.19$ & $29.03 \pm 0.50$ & $11.67 \pm 0.52$ & $67.42 \pm 3.35$ \\
Vivarium Control Encl, 8 mice & $17.56 \pm 0.37$ & $29.24 \pm 0.98$ & $11.69 \pm 0.82$ & $66.58 \pm 4.69$
\end{tabular}


Figure 26 (Experiment 2): Mean \pm S.E.M. starting body weight (day 1 ) and mean \pm S.E.M. ending body weight (day 30) (See also Table 27).
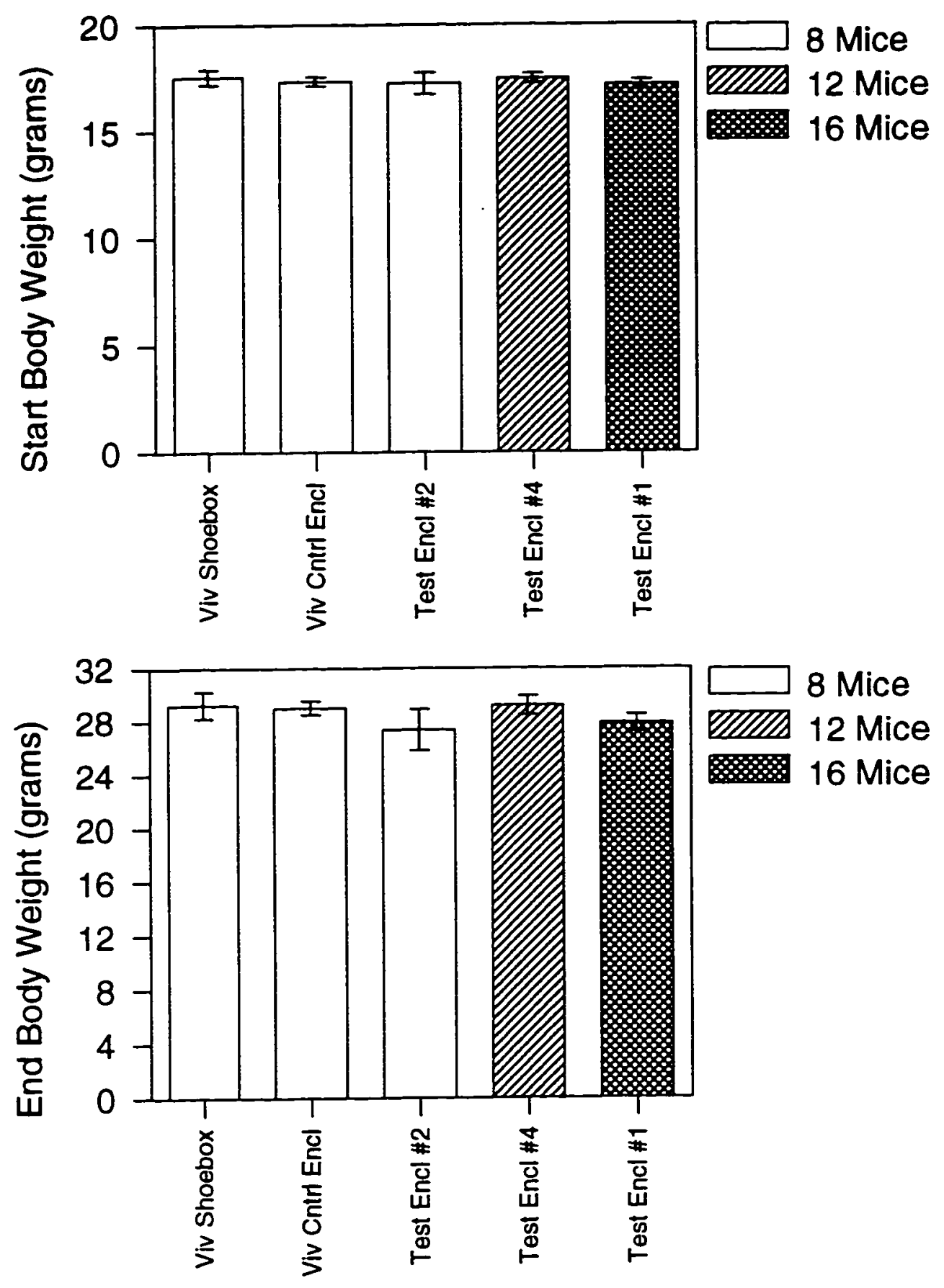
Figure 27 (Experiment 2): Mean \pm S.E.M. body weight gain and mean \pm S.E.M. per cent body weight gain (See also Table 27).
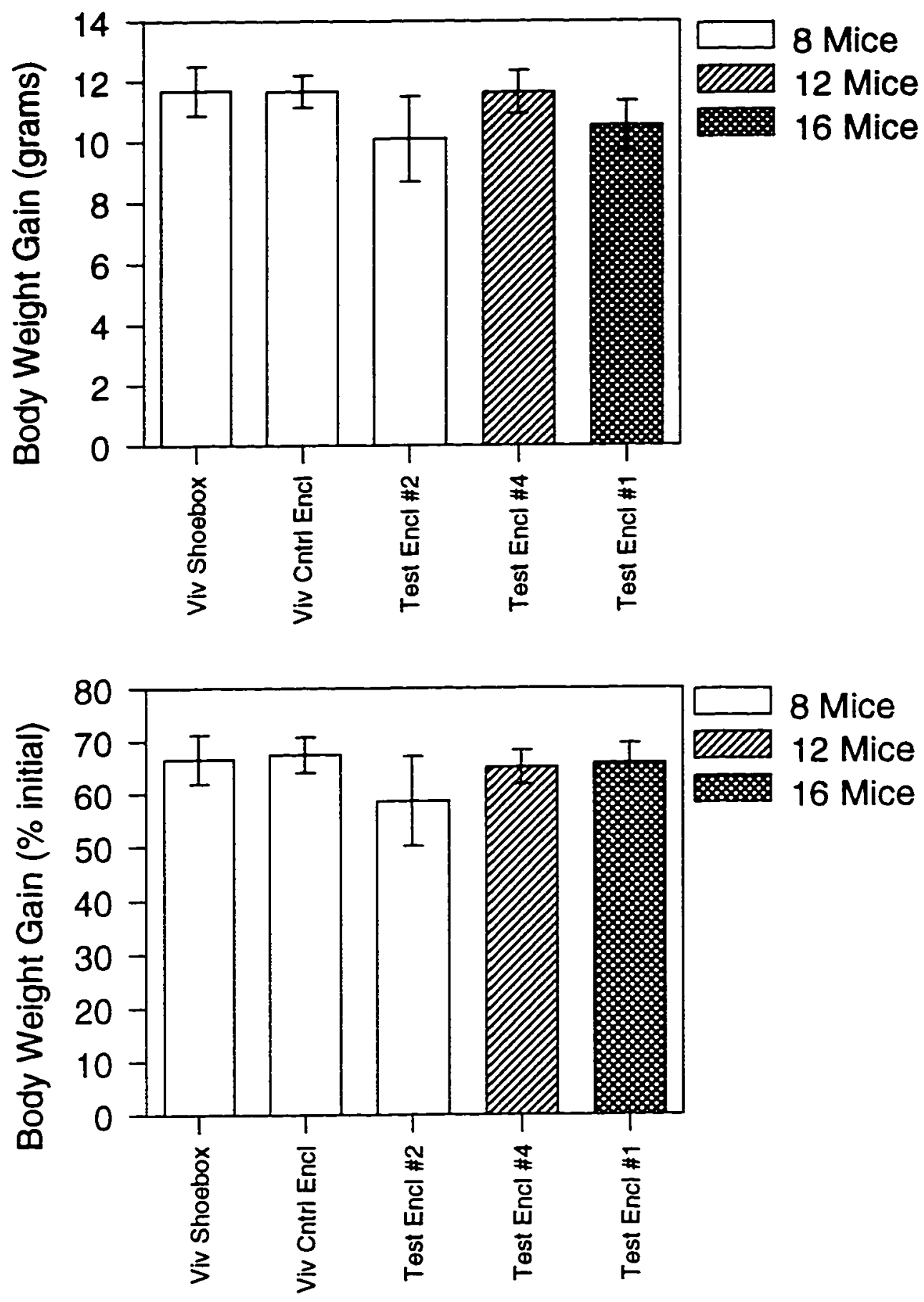
Table 28 (Experiment 2). Organ weights in grams and as a percentage of final body weight (BW)

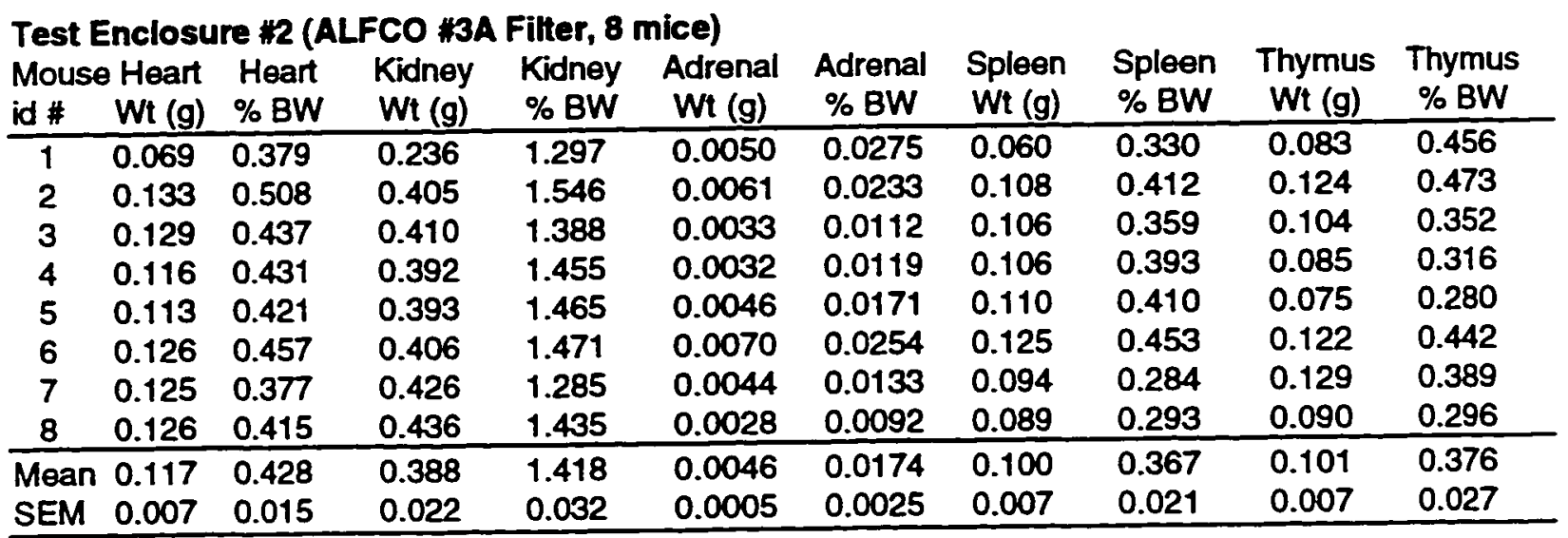

Test Enclosure \#4 (ALFCO \#3A Filter, 12 mice)

\begin{tabular}{|c|c|c|c|c|c|c|c|c|c|c|}
\hline $\begin{array}{l}\text { Mouse } \\
\text { id \# }\end{array}$ & $\begin{array}{l}\text { Heart } \\
\text { Wt (g) }\end{array}$ & $\begin{array}{l}\text { Heart } \\
\% \text { BW }\end{array}$ & $\begin{array}{l}\text { Kidney } \\
\text { Wt }(g)\end{array}$ & $\begin{array}{l}\text { Kidney } \\
\% \text { BW }\end{array}$ & $\begin{array}{c}\text { Adrenal } \\
\text { Wt (g) }\end{array}$ & $\begin{array}{l}\text { Adrenal } \\
\% \text { BW }\end{array}$ & $\begin{array}{l}\text { Spleen } \\
\text { Wt (g) }\end{array}$ & $\begin{array}{l}\text { Spleen } \\
\% \text { BW }\end{array}$ & $\begin{array}{l}\text { Thymus } \\
\text { Wt (g) }\end{array}$ & $\begin{array}{l}\text { Thymus } \\
\% \text { BW }\end{array}$ \\
\hline 9 & 0.117 & 0.422 & 0.386 & 1.392 & 0.0054 & 0.0195 & 0.088 & 0.317 & 0.079 & 0.285 \\
\hline 10 & 0.132 & 0.492 & 0.496 & 1.848 & 0.0005 & 0.0019 & 0.110 & 0.410 & 0.131 & 0.488 \\
\hline 11 & 0.143 & 0.511 & 0.470 & 1.680 & 0.0047 & 0.0168 & 0.101 & 0.361 & 0.108 & 0.386 \\
\hline 12 & 0.136 & 0.446 & 0.488 & 1.601 & 0.0068 & 0.0223 & 0.148 & 0.485 & 0.099 & 0.325 \\
\hline 13 & 0.151 & 0.482 & 0.393 & 1.255 & 0.0067 & 0.0214 & 0.161 & 0.514 & 0.086 & 0.275 \\
\hline 14 & 0.108 & 0.400 & 0.406 & 1.505 & 0.0065 & 0.0241 & 0.082 & 0.304 & 0.080 & 0.297 \\
\hline 15 & 0.142 & 0.471 & 0.480 & 1.592 & 0.0051 & 0.0169 & 0.118 & 0.391 & 0.088 & 0.292 \\
\hline 16 & 0.148 & 0.463 & 0.502 & 1.569 & 0.0052 & 0.0163 & 0.106 & 0.331 & 0.104 & 0.325 \\
\hline 17 & 0.120 & 0.447 & 0.374 & 1.394 & 0.0071 & 0.0265 & 0.083 & 0.309 & 0.089 & 0.332 \\
\hline 18 & 0.135 & 0.438 & 0.468 & 1.519 & 0.0042 & 0.0136 & 0.130 & 0.422 & 0.101 & 0.328 \\
\hline 19 & 0.114 & 0.423 & 0.403 & 1.496 & 0.0034 & 0.0126 & 0.105 & 0.390 & 0.088 & 0.327 \\
\hline 20 & 0.128 & 0.463 & 0.346 & 1.251 & 0.0048 & 0.0174 & 0.108 & 0.390 & 0.059 & 0.213 \\
\hline Mean & 0.131 & 0.455 & 0.434 & 1.508 & 0.0050 & 0.0174 & 0.112 & 0.385 & 0.093 & 0.323 \\
\hline SEM & 0.004 & 0.009 & 0.016 & 0.049 & 0.0005 & 0.0018 & 0.007 & 0.019 & 0.005 & 0.019 \\
\hline
\end{tabular}




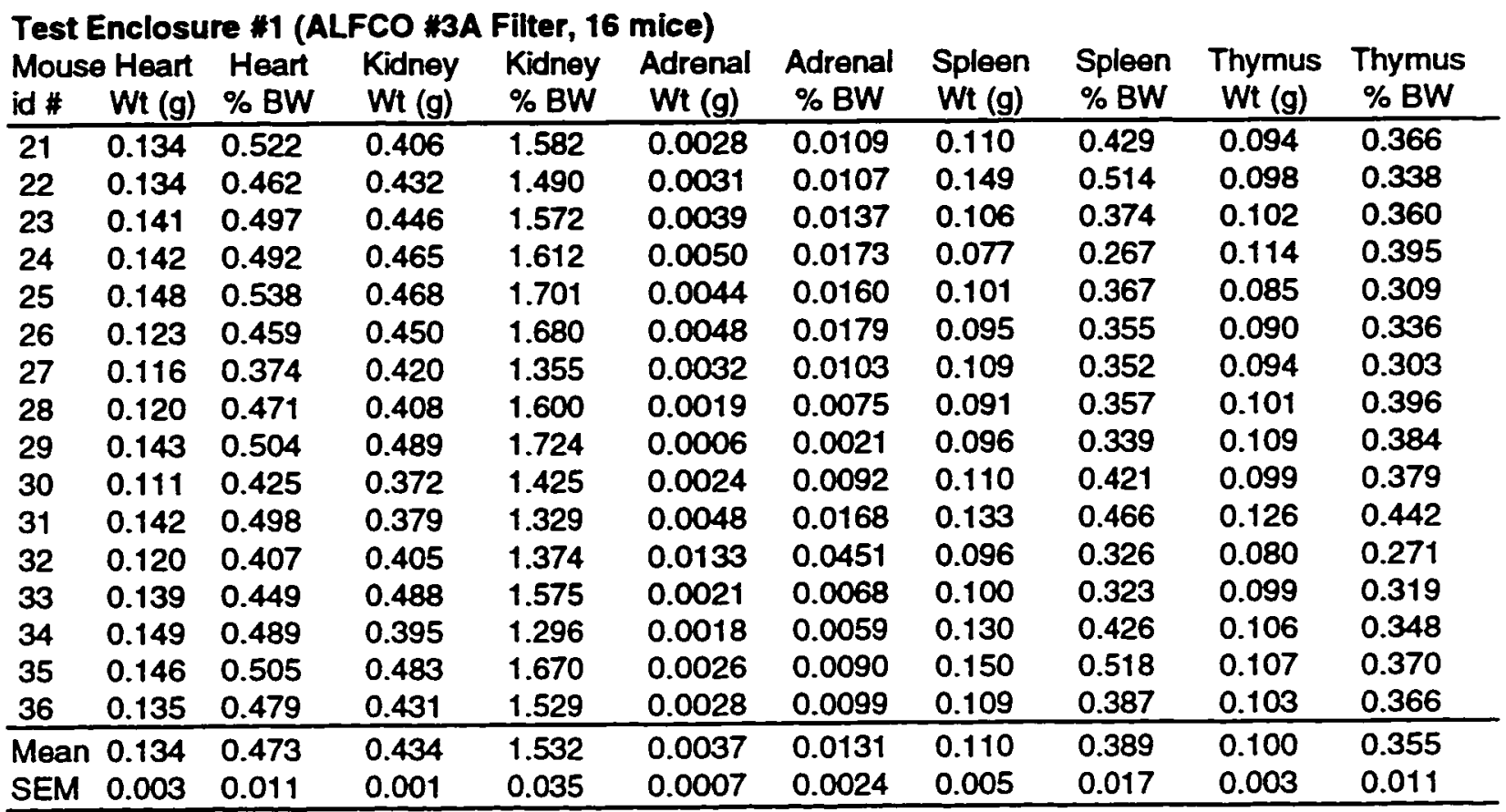

\begin{tabular}{|c|c|c|c|c|c|c|c|c|c|c|}
\hline \multicolumn{11}{|c|}{ Vivarium Control Enclosure ( 8 mice) } \\
\hline $\begin{array}{l}\text { Mouse } \\
\text { id \# }\end{array}$ & $\begin{array}{l}\text { Heart } \\
\text { Wt (g) }\end{array}$ & $\begin{array}{l}\text { Heart } \\
\% \text { BW }\end{array}$ & $\begin{array}{l}\text { Kidney } \\
\text { Wt (g) }\end{array}$ & $\begin{array}{l}\text { Kidney } \\
\% \text { BW }\end{array}$ & $\begin{array}{l}\text { Adrenal } \\
\text { Wt (g) }\end{array}$ & $\begin{array}{c}\text { Adrenal } \\
\% \text { BW }\end{array}$ & $\begin{array}{l}\text { Spleen } \\
\text { Wt (g) }\end{array}$ & $\begin{array}{l}\text { Spleen } \\
\% \text { BW }\end{array}$ & $\begin{array}{l}\text { Thymus } \\
\text { Wt (g) }\end{array}$ & $\begin{array}{l}\text { Thymus } \\
\% \text { BW }\end{array}$ \\
\hline 37 & 0.146 & 0.488 & 0.424 & 1.417 & 0.0041 & 0.0137 & 0.106 & 0.354 & 0.089 & 0.297 \\
\hline 38 & 0.140 & 0.464 & 0.496 & 1.643 & 0.0024 & 0.0079 & 0.111 & 0.368 & 0.090 & 0.298 \\
\hline 39 & 0.143 & 0.480 & 0.485 & 1.626 & 0.0036 & 0.0121 & 0.120 & 0.402 & 0.162 & 0.543 \\
\hline 40 & 0.146 & 0.563 & 0.386 & 1.489 & 0.0069 & 0.0266 & 0.108 & 0.417 & 0.124 & 0.478 \\
\hline 41 & 0.152 & 0.506 & 0.467 & 1.554 & 0.0047 & 0.0156 & 0.142 & 0.473 & 0.060 & 0.200 \\
\hline 42 & 0.120 & 0.422 & 0.382 & 1.344 & 0.0054 & 0.0190 & 0.096 & 0.338 & 0.151 & 0.531 \\
\hline 43 & 0.160 & 0.545 & 0.441 & 1.503 & 0.0030 & 0.0102 & 0.112 & 0.382 & 0.110 & 0.375 \\
\hline 44 & 0.130 & 0.456 & 0.464 & 1.627 & 0.0035 & 0.0123 & 0.109 & 0.382 & 0.101 & 0.354 \\
\hline Mean & 0.142 & 0.490 & 0.443 & 1.525 & 0.0042 & 0.0147 & 0.113 & 0.389 & 0.111 & 0.385 \\
\hline SEM & 0.004 & 0.016 & 0.015 & 0.038 & 0.0005 & 0.0021 & 0.005 & 0.015 & 0.012 & 0.043 \\
\hline
\end{tabular}




\begin{tabular}{|c|c|c|c|c|c|c|c|c|c|c|}
\hline $\begin{array}{l}\text { Vivari } \\
\text { Mouse } \\
\text { id \# }\end{array}$ & $\begin{array}{l}\text { um She } \\
\text { o Heart } \\
\text { Wt (g) }\end{array}$ & $\begin{array}{l}\text { Box C } \\
\text { Heart } \\
\% \text { BW }\end{array}$ & $\begin{array}{l}\text { ntrol, } 8 \\
\text { Kidney } \\
\text { Wt (g) }\end{array}$ & $\begin{array}{l}\text { Kidney } \\
\% \text { BW }\end{array}$ & $\begin{array}{l}\text { Adrenal } \\
\text { Wt (g) }\end{array}$ & $\begin{array}{l}\text { Adrenal } \\
\% \text { BW }\end{array}$ & $\begin{array}{l}\text { Spleen } \\
\text { Wt (g) }\end{array}$ & $\begin{array}{l}\text { Spleen } \\
\% \text { BW }\end{array}$ & $\begin{array}{l}\text { Thymus } \\
\text { Wt (g) }\end{array}$ & $\begin{array}{l}\text { Thymus } \\
\% \text { BW }\end{array}$ \\
\hline$\overline{45}$ & 0.156 & 0.517 & 0.404 & 1.338 & 0.0052 & 0.0172 & 0.086 & 0.285 & 0.109 & 0.361 \\
\hline 46 & 0.132 & 0.456 & 0.483 & 1.668 & 0.0035 & 0.0121 & 0.116 & 0.401 & 0.166 & 0.573 \\
\hline 47 & 0.148 & 0.476 & 0.446 & 1.434 & 0.0047 & 0.0151 & 0.085 & 0.273 & 0.134 & 0.431 \\
\hline 48 & 0.142 & 0.437 & 0.381 & 1.172 & 0.0032 & 0.0098 & 0.099 & 0.304 & 0.135 & 0.415 \\
\hline 49 & 0.180 & 0.597 & 0.464 & 1.538 & 0.0033 & 0.0109 & 0.080 & 0.265 & 0.127 & 0.421 \\
\hline 50 & 0.155 & 0.585 & 0.474 & 1.789 & 0.0045 & 0.0170 & 0.079 & 0.298 & 0.088 & 0.332 \\
\hline 51 & 0.126 & 0.527 & 0.333 & 1.393 & 0.0039 & 0.0163 & 0.069 & 0.289 & 0.122 & 0.510 \\
\hline 52 & 0.139 & 0.454 & 0.439 & 1.434 & 0.0030 & 0.0098 & 0.113 & 0.369 & 0.126 & 0.411 \\
\hline$\overline{\mathrm{Mea}}$ & 0.147 & 0.506 & 0.428 & 1.471 & 0.0039 & 0.0135 & 0.091 & 0.311 & 0.126 & 0.432 \\
\hline SEM & 0.006 & 0.022 & 0.018 & 0.068 & 0.0003 & 0.0011 & 0.006 & 0.017 & 0.008 & 0.027 \\
\hline
\end{tabular}

Table 29 (Experiment 2). Mean \pm S.E.M organ weights by cage.

\begin{tabular}{lcccc} 
& $\begin{array}{c}\text { Heart } \\
\text { Wt }(\mathrm{g})\end{array}$ & $\begin{array}{c}\text { Heart } \\
\text { Wt (\% Final Wt) }\end{array}$ & $\begin{array}{c}\text { Kidney } \\
\text { Wt }(\mathrm{g})\end{array}$ & $\begin{array}{c}\text { Kidney } \\
\text { Wt (\% Final Wt) }\end{array}$ \\
\hline Test Enclosure \#2, 8 mice & $0.117 \pm 0.0073$ & $0.428 \pm 0.0149$ & $0.388 \pm 0.0224$ & $1.418 \pm 0.0317$ \\
Test Enclosure \#4, 12 mice & $0.131 \pm 0.0040$ & $0.455 \pm 0.0092$ & $0.434 \pm 0.0158$ & $1.508 \pm 0.0494$ \\
Test Enclosure \#1, 16 mice & $0.134 \pm 0.0030$ & $0.473 \pm 0.0108$ & $0.434 \pm 0.0095$ & $1.532 \pm 0.0348$ \\
Vivarium Shoe Box Cntrl, 8 mice & $0.147 \pm 0.0060$ & $0.506 \pm 0.0215$ & $0.428 \pm 0.0183$ & $1.471 \pm 0.0682$ \\
Vivarium Control Encl, 8 mice & $0.142 \pm 0.0044$ & $0.490 \pm 0.0165$ & $0.443 \pm 0.0152$ & $1.525 \pm 0.0382$
\end{tabular}

\begin{tabular}{lcccc} 
& $\begin{array}{c}\text { Adrenal } \\
\text { Wt }(\mathrm{g})\end{array}$ & $\begin{array}{c}\text { Adrenal } \\
\text { Wt }(\% \text { Final Wt) }\end{array}$ & $\begin{array}{c}\text { Spleen } \\
\text { Wt }(\mathrm{g})\end{array}$ & $\begin{array}{c}\text { Spleen } \\
\text { Wt }(\% \text { Final Wt) }\end{array}$ \\
\hline Test Enclosure \#2, 8 mice & $0.0046 \pm 0.00052$ & $0.017 \pm 0.0025$ & $0.100 \pm 0.0068$ & $0.367 \pm 0.0215$ \\
Test Enclosure \#4, 12 mice & $0.0050 \pm 0.00053$ & $0.017 \pm 0.0018$ & $0.112 \pm 0.0071$ & $0.386 \pm 0.0193$ \\
Test Enclosure \#1, 16 mice & $0.0037 \pm 0.00071$ & $0.013 \pm 0.0024$ & $0.110 \pm 0.0051$ & $0.389 \pm 0.0174$ \\
Vivarium Shoe Box Cntrl, 8 mice & $0.0039 \pm 0.00028$ & $0.014 \pm 0.0011$ & $0.091 \pm 0.0060$ & $0.310 \pm 0.0171$ \\
Vivarium Control Encl, 8 mice & $0.0042 \pm 0.00051$ & $0.015 \pm 0.0021$ & $0.113 \pm 0.0048$ & $0.389 \pm 0.0148$
\end{tabular}

\begin{tabular}{lcc} 
& $\begin{array}{c}\text { Thymus } \\
\text { Wt }(\mathrm{g})\end{array}$ & $\begin{array}{c}\text { Thymus } \\
\text { Wt }(\% \text { Final Wt })\end{array}$ \\
\hline Test Enclosure \#2, 8 mice & $0.102 \pm 0.0075$ & $0.376 \pm 0.0268$ \\
Test Enclosure \#4, 12 mice & $0.093 \pm 0.0052$ & $0.323 \pm 0.0192$ \\
Test Enclosure \#1, 16 mice & $0.100 \pm 0.0028$ & $0.355 \pm 0.0105$ \\
Vivarium Shoe Box Cntrl, 8 mice & $0.126 \pm 0.0079$ & $0.432 \pm 0.0274$ \\
Vivarium Control Encl, 8 mice & $0.111 \pm 0.0119$ & $0.385 \pm 0.0435$
\end{tabular}


Figure 28 (Experiment 2). Mean \pm S.E.M. heart weight. and mean \pm S.E.M. heart weight as a percent of final body weight. (See also Table 29)
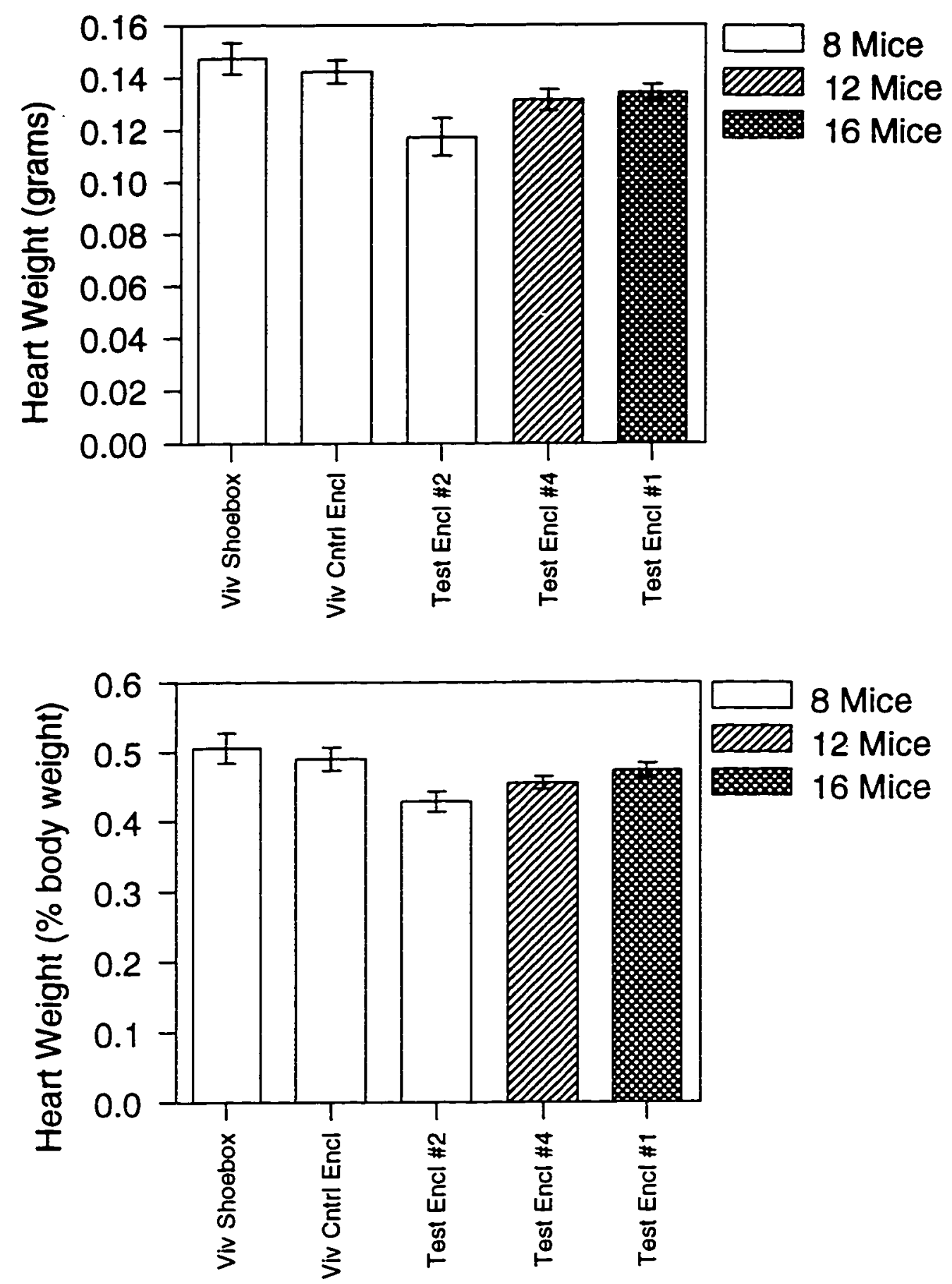
Figure 29 (Experiment 2). Mean \pm S.E.M. kidney weight. and mean \pm S.E.M. kidney weight as a percent of final body weight (See also Table 29).
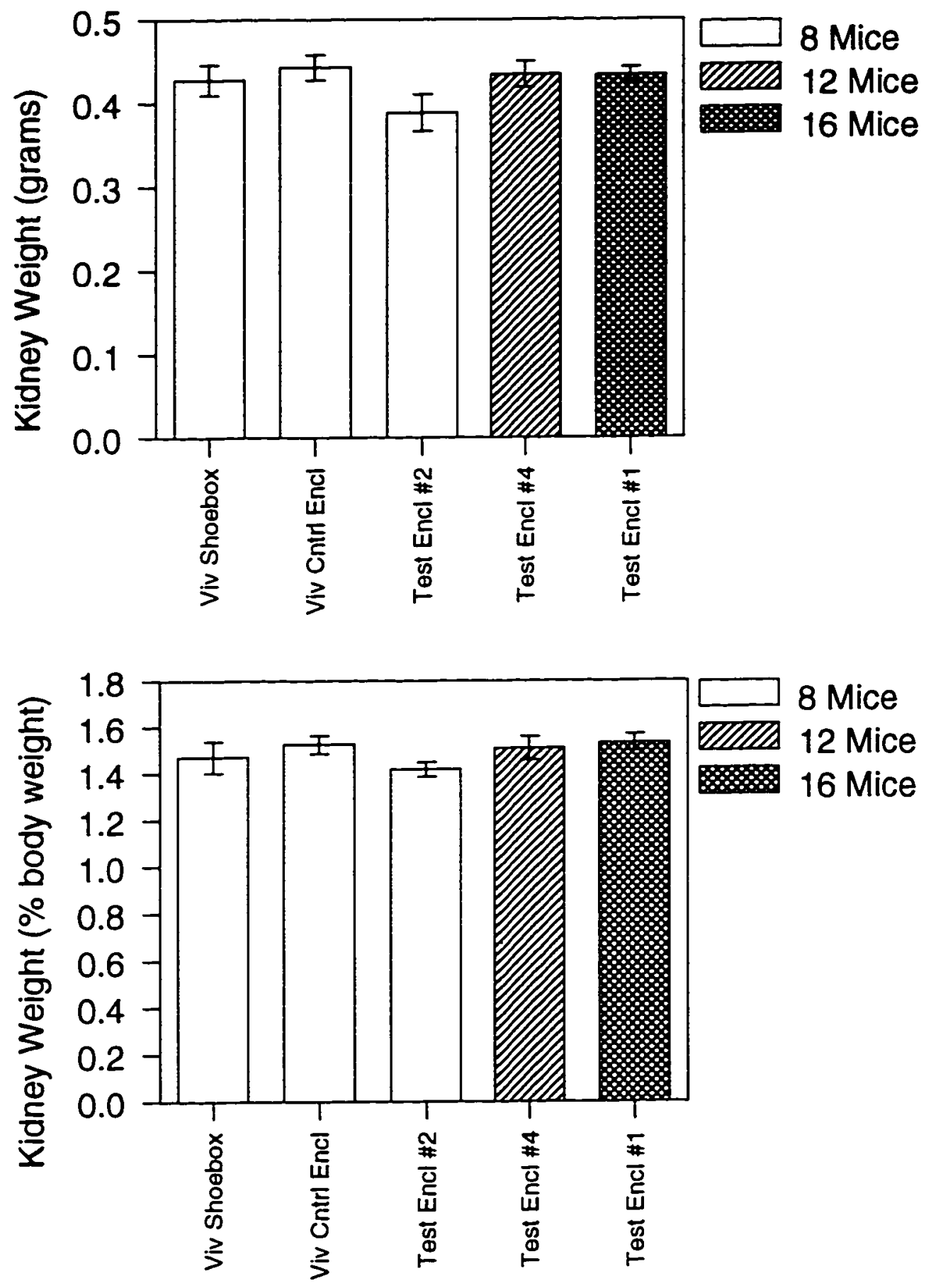
Figure 30 (Experiment 2). Mean \pm S.E.M. bilateral adrenal weight. and mean \pm S.E.M. bilateral adrenal weight as a percent of final body weight (See also Table 29).
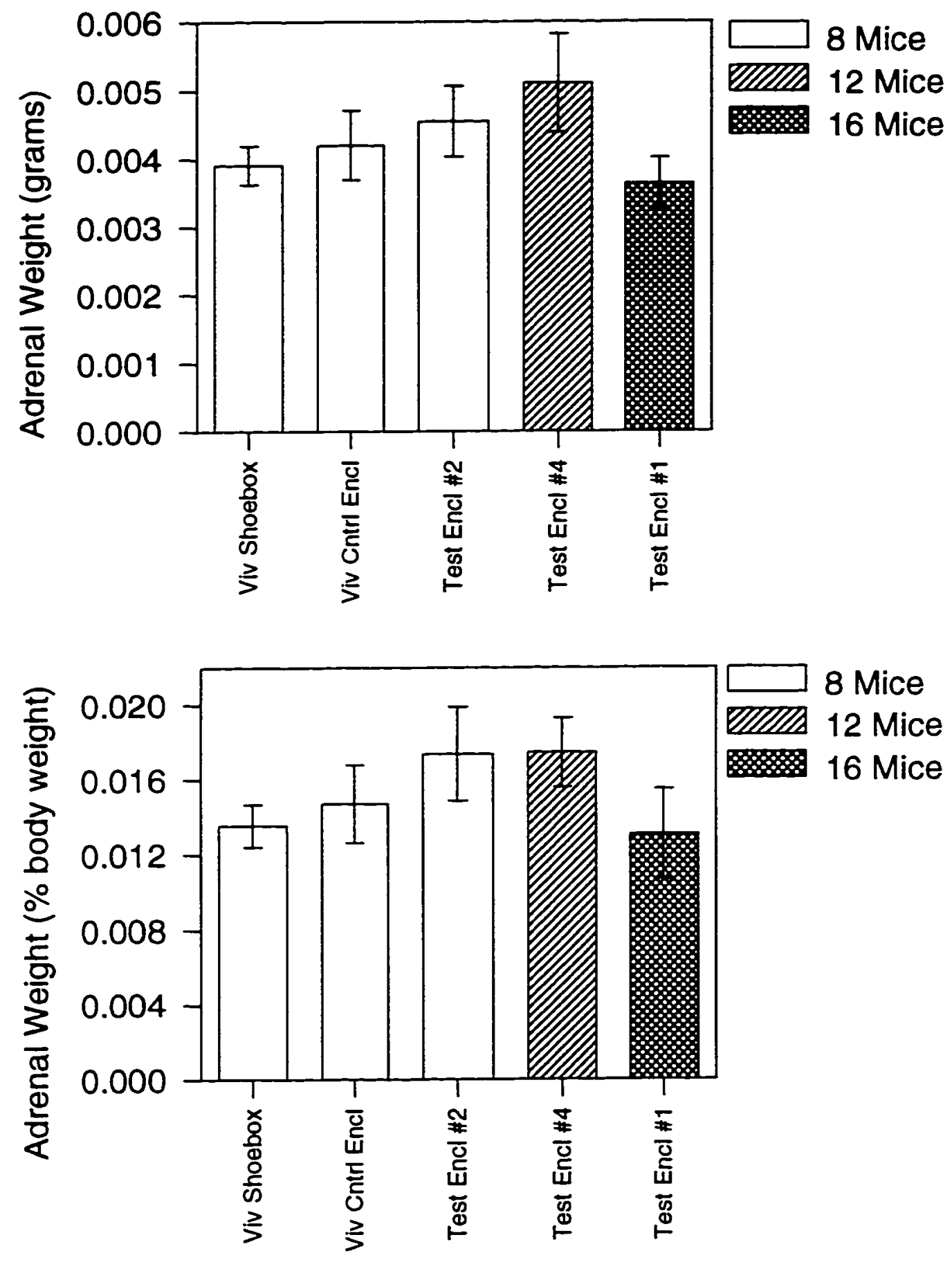
Figure 31 (Experiment 2). Mean \pm S.E.M. spleen weight. and mean \pm S.E.M. spleen weight as a percent of final body weight (See also 29).
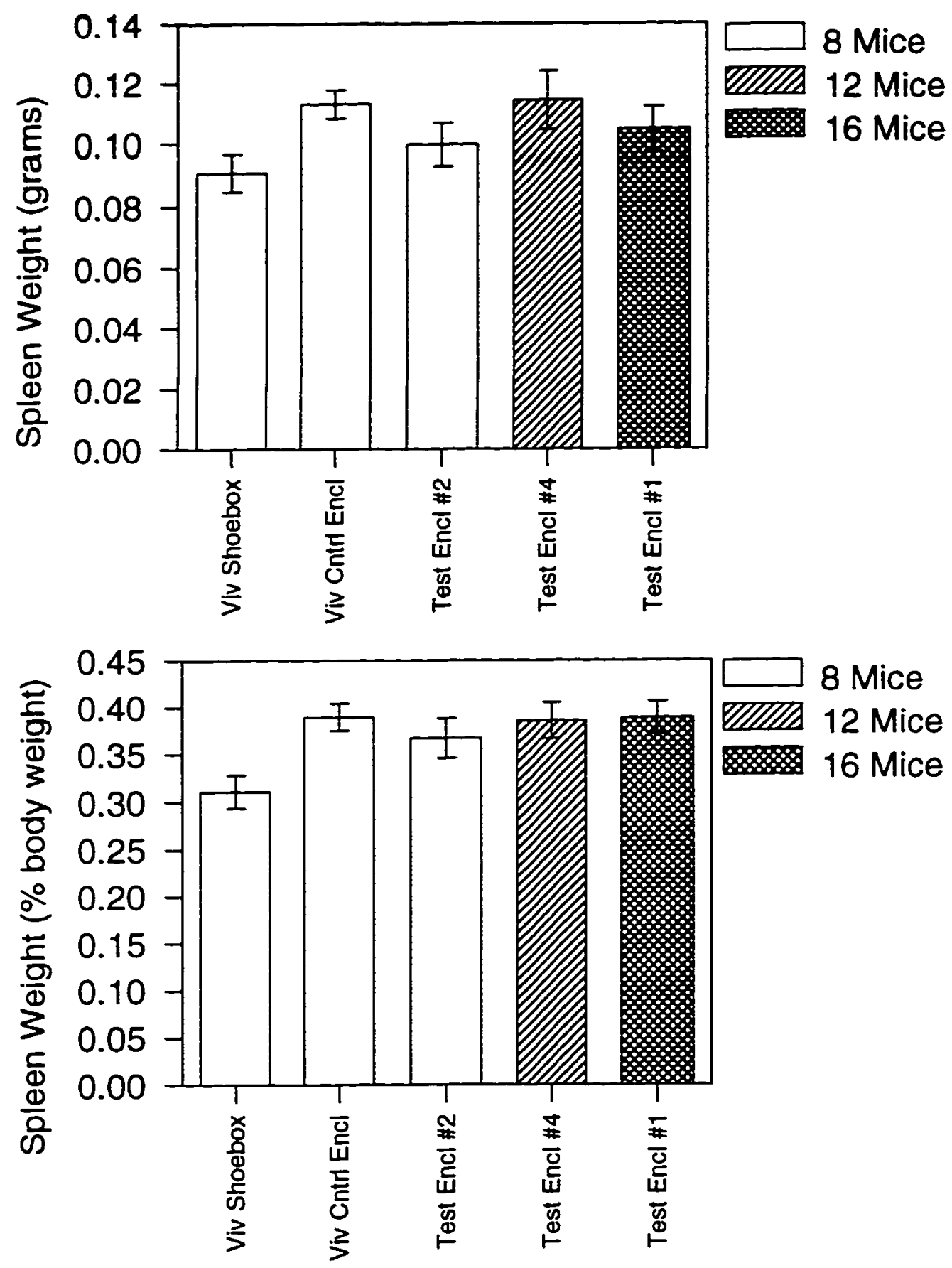
Figure 32 (Experiment 2). Mean \pm S.E.M. thymus weight, and mean \pm S.E.M. thymus weight as a percent of final body weight (See also 29).
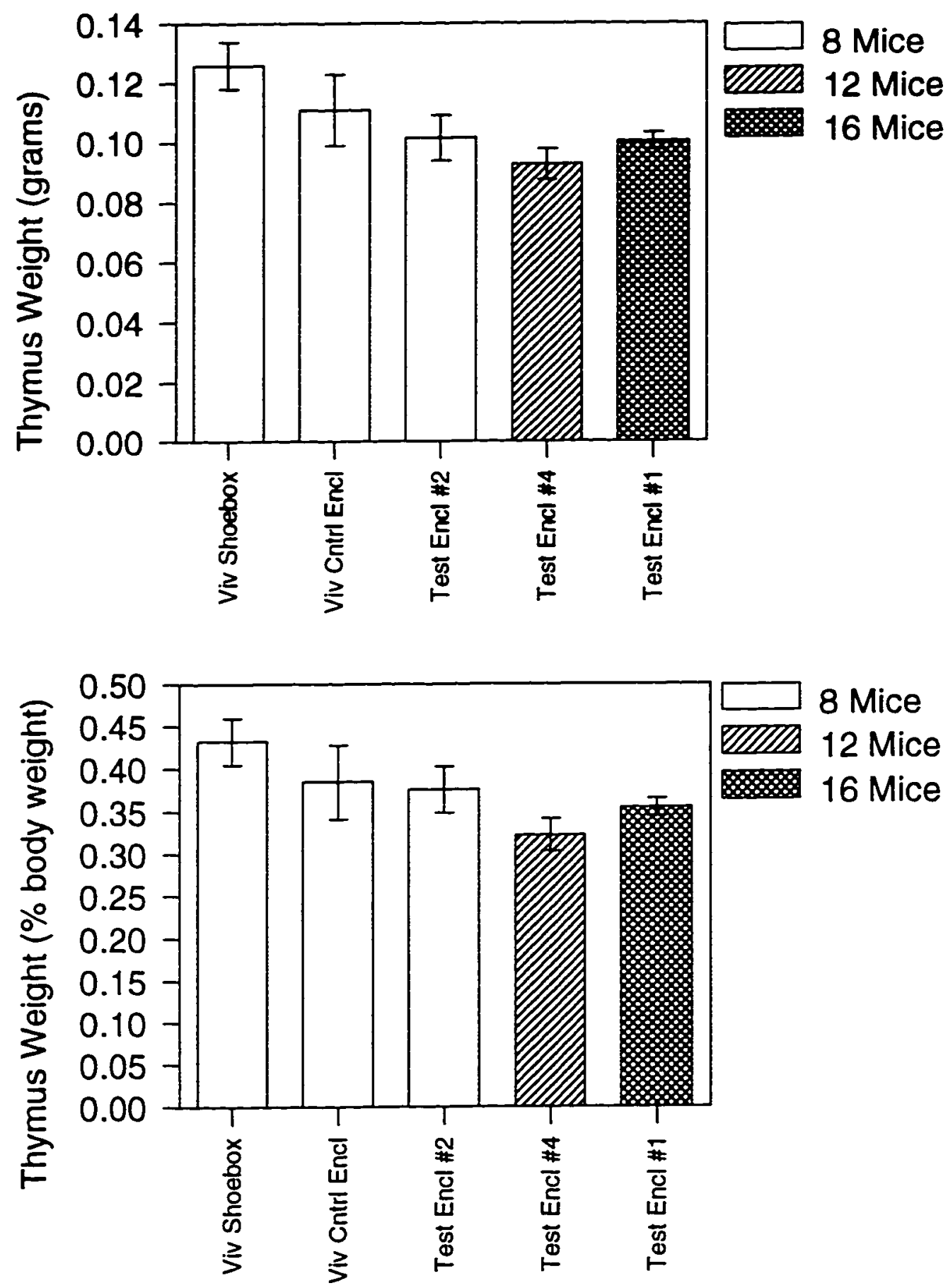
Table 30 (Experiment 2). Total white blood cell count (WBC), plasma corticosterone, plasma protein, eosinophil (EOS) count, basophil (BAS) count, neutrophil (NEU) count, monocyte (MON) count, lymphocyte (LYM) count, and neutrophil to lymphocyte ratio $(\mathrm{N} / \mathrm{L})$

\begin{tabular}{|c|c|c|c|c|c|c|c|c|c|}
\hline $\begin{array}{l}\text { Mouse } \\
\text { id \# }\end{array}$ & $\begin{array}{c}\text { Plasma } \\
\text { Corticosterone } \\
\mu \mathrm{g} / 100 \mathrm{ml}\end{array}$ & $\begin{array}{l}\text { Plasma } \\
\text { Protein } \\
g / d l\end{array}$ & $\begin{array}{c}\text { Total } \\
\text { WBC } \\
\text { cells } \times 10^{3} / \text { ul }\end{array}$ & $\begin{array}{c}\text { EOS } \\
\text { count } \\
\% \text { WBC }\end{array}$ & $\begin{array}{c}\text { BAS } \\
\text { count } \\
\% \text { WBC }\end{array}$ & $\begin{array}{c}\text { NEU } \\
\text { count } \\
\% \text { WBC }\end{array}$ & $\begin{array}{r}\text { MON } \\
\text { count } \\
\% \text { WBC } \\
\end{array}$ & $\begin{array}{c}\text { LYM } \\
\text { count } \\
\% \text { WBC } \\
\end{array}$ & $\begin{array}{l}\mathrm{N} / \\
\text { count }\end{array}$ \\
\hline 1 & 2.49 & 1.20 & 8.63 & 0 & 0 & 1 & 2 & 97 & 0.010 \\
\hline 2 & 12.56 & 1.10 & 11.00 & 3 & 0 & 3 & 3 & 84 & 0.036 \\
\hline 3 & 16.55 & 1.22 & 8.13 & n.d. & n.d. & n.d. & n.d. & n.d. & n.d. \\
\hline 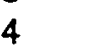 & 15.73 & 1.29 & 15.75 & 5 & 0 & 1 & 1 & 87 & 0.011 \\
\hline 5 & 3.49 & 0.24 & 6.25 & 0 & 0 & 3 & 1 & 96 & 0.031 \\
\hline 6 & 26.52 & 1.14 & 9.13 & 1 & 0 & 6 & 1 & 92 & 0.065 \\
\hline 7 & 7.10 & 1.22 & 11.25 & 2 & 0 & 10 & 4 & 84 & 0.119 \\
\hline 8 & 16.68 & 1.23 & 8.00 & 4 & 0 & 15 & 5 & 76 & 0.197 \\
\hline Mes & 12.64 & 1.08 & 9.77 & 2 & 0 & 6 & 2 & 88 & 0.067 \\
\hline S.E.M & 2.84 & 0.12 & 1.03 & 1 & 0 & 2 & 1 & 3 & 0.026 \\
\hline
\end{tabular}

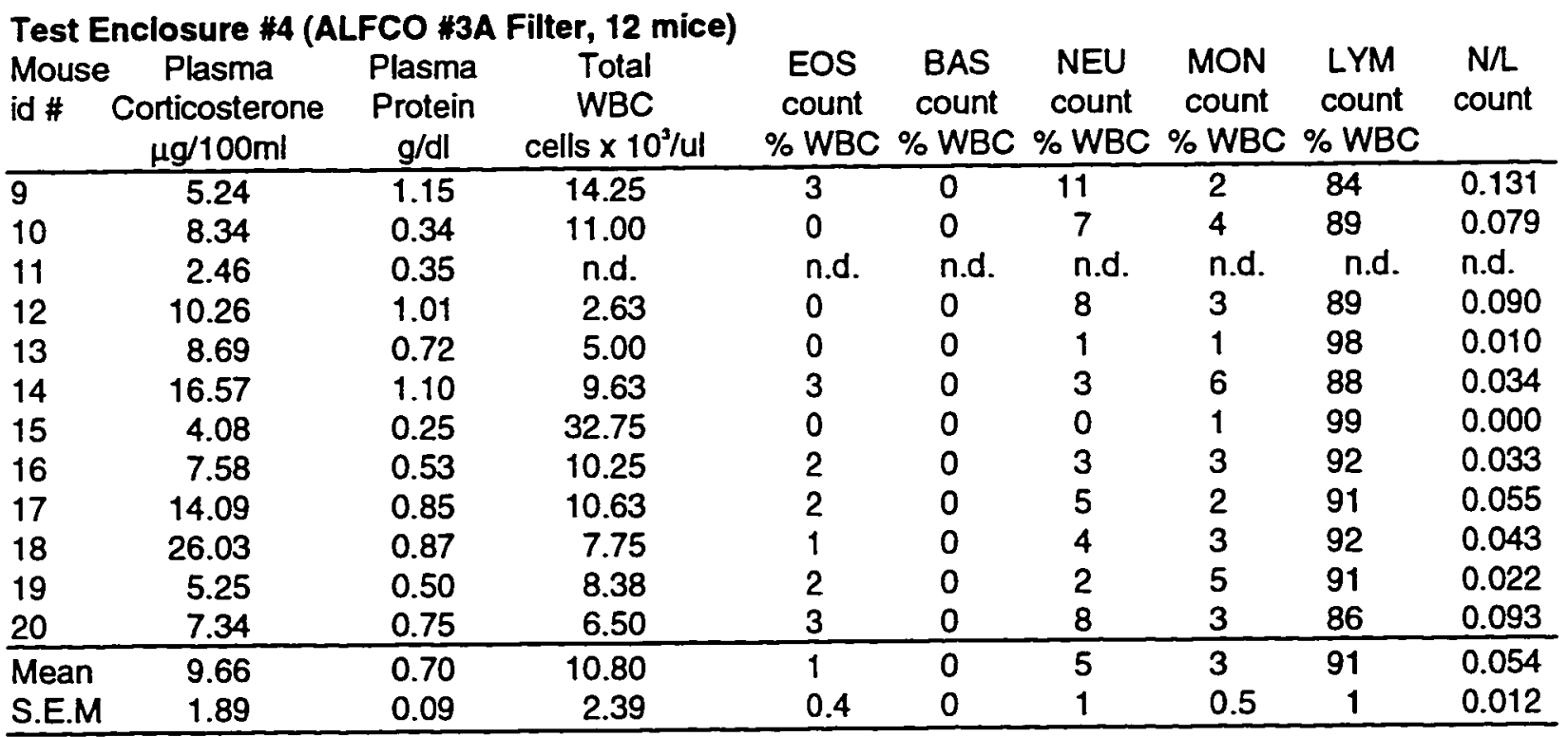




\begin{tabular}{|c|c|c|c|c|c|c|c|c|c|}
\hline $\begin{array}{l}\text { Mouse } \\
\text { id \# }\end{array}$ & $\begin{array}{c}\text { Plasma } \\
\text { Corticosterone } \\
\mu \mathrm{g} / 100 \mathrm{ml}\end{array}$ & $\begin{array}{c}\text { Plasma } \\
\text { Protein } \\
\text { g/dl } \\
\end{array}$ & $\begin{array}{c}\text { Total } \\
\text { WBC } \\
\text { cells } \times 10^{3} / \mathrm{ul} \\
\end{array}$ & $\begin{array}{c}\text { EOS } \\
\text { count } \\
\% \text { WBC }\end{array}$ & $\begin{array}{c}\text { BAS } \\
\text { count } \\
\% \text { WBC } \\
\end{array}$ & $\begin{array}{c}\text { NEU } \\
\text { count } \\
\% \text { WBC } \\
\end{array}$ & $\begin{array}{c}\text { MON } \\
\text { count } \\
\% \text { WBC } \\
\end{array}$ & $\begin{array}{c}\text { LYM } \\
\text { count } \\
\% \text { WBC }\end{array}$ & $\begin{array}{c}N / L \\
\text { count }\end{array}$ \\
\hline$\overline{21}$ & 5.67 & 0.46 & 14.50 & 2 & 0 & 2 & 4 & 92 & 0.022 \\
\hline 22 & 28.47 & 1.38 & 13.38 & 5 & 0 & 3 & 2 & 90 & 0.033 \\
\hline 23 & 6.42 & 0.40 & 27.63 & 0 & 0 & 2 & 4 & 94 & 0.021 \\
\hline 24 & 15.67 & 1.23 & 7.50 & 2 & 0 & 7 & 1 & 90 & 0.078 \\
\hline 25 & 16.63 & 1.12 & 9.88 & 1 & 0 & 8 & 2 & 89 & 0.090 \\
\hline 26 & 8.51 & 1.21 & 9.88 & 2 & 0 & 11 & 3 & 84 & 0.131 \\
\hline 27 & 15.85 & 0.66 & n.d. & n.d. & n.d. & n.d. & n.d. & n.d. & n.d. \\
\hline 28 & 21.17 & 1.08 & 13.75 & 3 & 0 & 5 & 2 & 90 & 0.056 \\
\hline 29 & 9.96 & 1.26 & 20.38 & 2 & 0 & 11 & 0 & 87 & 0.126 \\
\hline 30 & 14.13 & 0.66 & 9.50 & 2 & 0 & 4 & 4 & 90 & 0.044 \\
\hline 31 & 13.39 & 1.17 & 19.38 & 1 & 0 & 6 & 5 & 88 & 0.068 \\
\hline 32 & 9.76 & 0.45 & n.d. & n.d. & n.d. & n.d. & n.d. & n.d. & n.d. \\
\hline 33 & 13.19 & 1.38 & 10.38 & 5 & 0 & 8 & 3 & 84 & 0.095 \\
\hline 34 & 16.00 & 1.08 & 13.00 & 1 & 0 & 6 & 9 & 84 & 0.071 \\
\hline 35 & 5.77 & 0.81 & 14.50 & 1 & 0 & 0 & 1 & 98 & 0.000 \\
\hline 36 & 10.30 & 1.09 & 6.75 & 2 & 0 & 17 & 3 & 78 & 0.218 \\
\hline Mean & 13.18 & 0.97 & 13.60 & 2 & 0 & 6 & 3 & 88 & 0.075 \\
\hline S.E.M & 1.50 & 0.08 & 1.51 & 0.4 & 0 & 1 & 0.6 & 1 & 0.015 \\
\hline
\end{tabular}

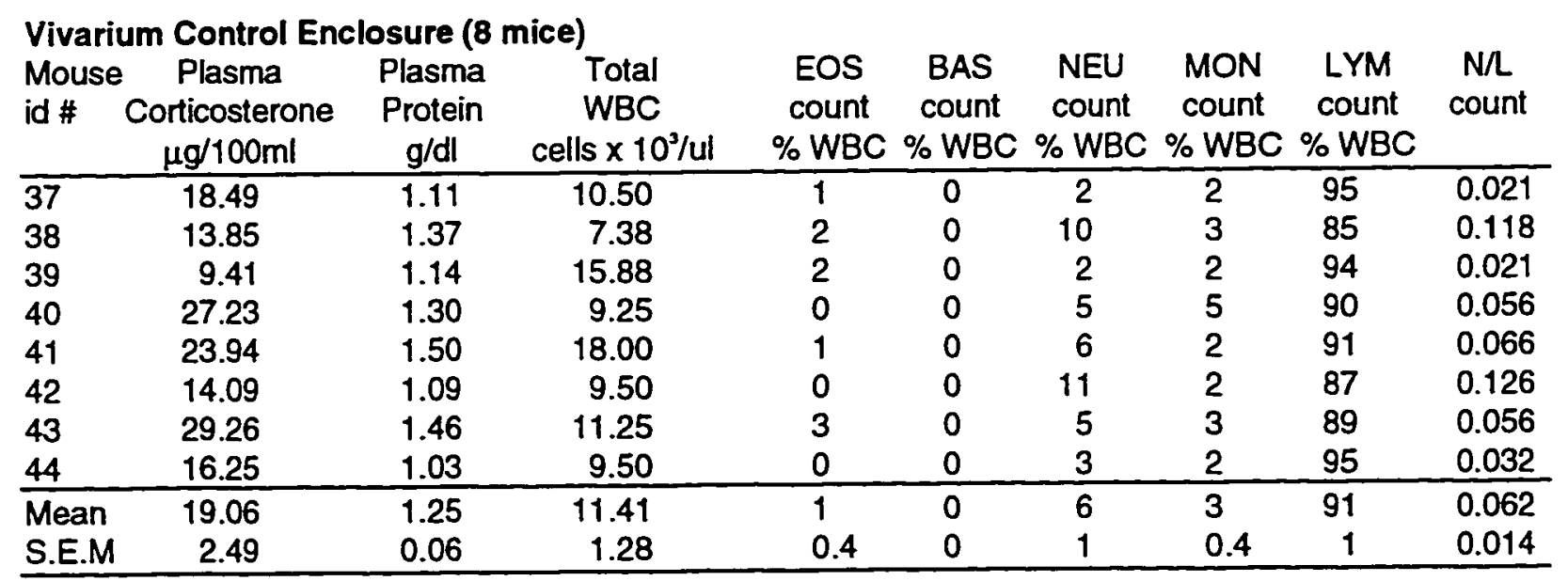




\begin{tabular}{|c|c|c|c|c|c|c|c|c|c|}
\hline \multicolumn{10}{|c|}{ Vivarium Shoe Box Control (8 mice) } \\
\hline $\begin{array}{l}\text { Mouse } \\
\text { id \# }\end{array}$ & $\begin{array}{l}\text { Plasma } \\
\text { Corticosterone } \\
\mu g / 100 \mathrm{ml}\end{array}$ & $\begin{array}{c}\text { Plasma } \\
\text { Protein } \\
\text { g/dl }\end{array}$ & $\begin{array}{c}\text { Total } \\
\text { WBC } \\
\text { cells } \times 10^{3} / \mathrm{ul}\end{array}$ & $\begin{array}{c}\text { EOS } \\
\text { count } \\
\% \text { WBC } \\
\end{array}$ & $\begin{array}{c}\text { BAS } \\
\text { count } \\
\% \text { WBC } \\
\end{array}$ & $\begin{array}{c}\text { NEU } \\
\text { count } \\
\% \text { WBC } \\
\end{array}$ & $\begin{array}{c}\text { MON } \\
\text { count } \\
\% \text { WBC } \\
\end{array}$ & $\begin{array}{c}\text { LYM } \\
\text { count } \\
\% \text { WBC } \\
\end{array}$ & $\begin{array}{c}\mathrm{N} / \mathrm{L} \\
\text { count }\end{array}$ \\
\hline$\overline{45}$ & 40.93 & 1.48 & 9.25 & 2 & 0 & 6 & 2 & 90 & 0.067 \\
\hline 46 & 20.71 & 0.99 & 10.38 & 1 & 0 & 7 & 5 & 85 & 0.082 \\
\hline 47 & 5.93 & 1.10 & 9.63 & 0 & 0 & 1 & 2 & 97 & 0.010 \\
\hline 48 & 10.85 & 1.19 & 6.00 & 0 & 0 & 14 & 5 & 81 & 0.173 \\
\hline 49 & 42.10 & 1.27 & 9.00 & 0 & 0 & 7 & 2 & 91 & 0.077 \\
\hline 50 & 27.44 & 1.06 & 12.25 & 3 & 0 & 5 & 1 & 91 & 0.055 \\
\hline 51 & 22.50 & 1.28 & 7.75 & 2 & 0 & 2 & 5 & 91 & 0.022 \\
\hline 52 & 12.94 & 1.40 & 8.75 & 2 & 0 & $\overline{5}$ & 3 & 90 & 0.056 \\
\hline$\overline{\text { Mean }}$ & 22.93 & 1.22 & 9.13 & 1 & 0 & 6 & 3 & 90 & 0.068 \\
\hline S.E.M & 4.73 & 0.06 & 0.65 & 0.4 & 0 & 1 & 1 & 2 & 0.017 \\
\hline
\end{tabular}

Table 31 (Experiment 2). Mean \pm S.E.M blood test values by cage.

\begin{tabular}{lccccc} 
& $\begin{array}{c}\text { Plasma } \\
\text { Corticosterone } \\
\mu \mathrm{g} / 100 \mathrm{ml}\end{array}$ & $\begin{array}{c}\text { Plasma } \\
\text { Protein } \\
\mathrm{g} / \mathrm{dl}\end{array}$ & $\begin{array}{c}\text { Total } \\
\text { WBC } \\
\text { cells } \times 10^{3} / \mathrm{ul}\end{array}$ & $\begin{array}{c}\text { EOS } \\
\text { count } \\
\% \text { WBC }\end{array}$ & $\begin{array}{c}\text { BAS } \\
\text { count }\end{array}$ \\
\hline Test Enclosure \#2, 8 mice & $12.64 \pm 2.84$ & $1.08 \pm 0.12$ & $9.77 \pm 1.03$ & $2 \pm 1.0$ & $0 \pm 0$ \\
Test Enclosure \#4, 12 mice & $9.66 \pm 1.89$ & $0.70 \pm 0.09$ & $10.80 \pm 2.39$ & $1 \pm 0.4$ & $0 \pm 0$ \\
Test Enclosure \#1, 16 mice & $13.18 \pm 1.50$ & $0.97 \pm 0.08$ & $13.60 \pm 1.51$ & $2 \pm 0.4$ & $0 \pm 0$ \\
Vivarium Shoe Box Cntrl, 8 mice & $19.06 \pm 2.49$ & $1.25 \pm 0.06$ & $11.41 \pm 1.28$ & $1 \pm 0.4$ & $0 \pm 0$ \\
Vivarium Control Encl, 8 mice & $22.93 \pm 4.73$ & $1.22 \pm 0.06$ & $9.13 \pm 0.65$ & $1 \pm 0.4$ & $0 \pm 0$
\end{tabular}

\begin{tabular}{lcccc} 
& $\begin{array}{c}\text { NEU } \\
\text { count } \\
\% \text { WBC }\end{array}$ & $\begin{array}{c}\text { MON } \\
\text { count } \\
\% \text { WBC }\end{array}$ & $\begin{array}{c}\text { LYM } \\
\text { count } \\
\% \text { WBC }\end{array}$ & $\begin{array}{c}\text { N/L } \\
\text { count } \\
\% \text { WBC }\end{array}$ \\
\hline Test Enclosure \#2, 8 mice & $6 \pm 2$ & $2 \pm 1.0$ & $88 \pm 3$ & $0.067 \pm 0.026$ \\
Test Enclosure \#4, 12 mice & $5 \pm 1$ & $3 \pm 0.5$ & $91 \pm 1$ & $0.054 \pm 0.012$ \\
Test Enclosure \#1, 16 mice & $6 \pm 1$ & $3 \pm 0.6$ & $88 \pm 1$ & $0.075 \pm 0.015$ \\
Vivarium Shoe Box Cntrl, 8 mice & $6 \pm 1$ & $3 \pm 0.4$ & $91 \pm 1$ & $0.062 \pm 0.014$ \\
Vivarium Control Encl, 8 mice & $6 \pm 1$ & $3 \pm 1.0$ & $90 \pm 2$ & $0.068 \pm 0.017$
\end{tabular}


Figure 33 (Experiment 2). Mean \pm S.E.M. plasma corticosterone and mean \pm S.E.M. plasma protein (See also Table 31).
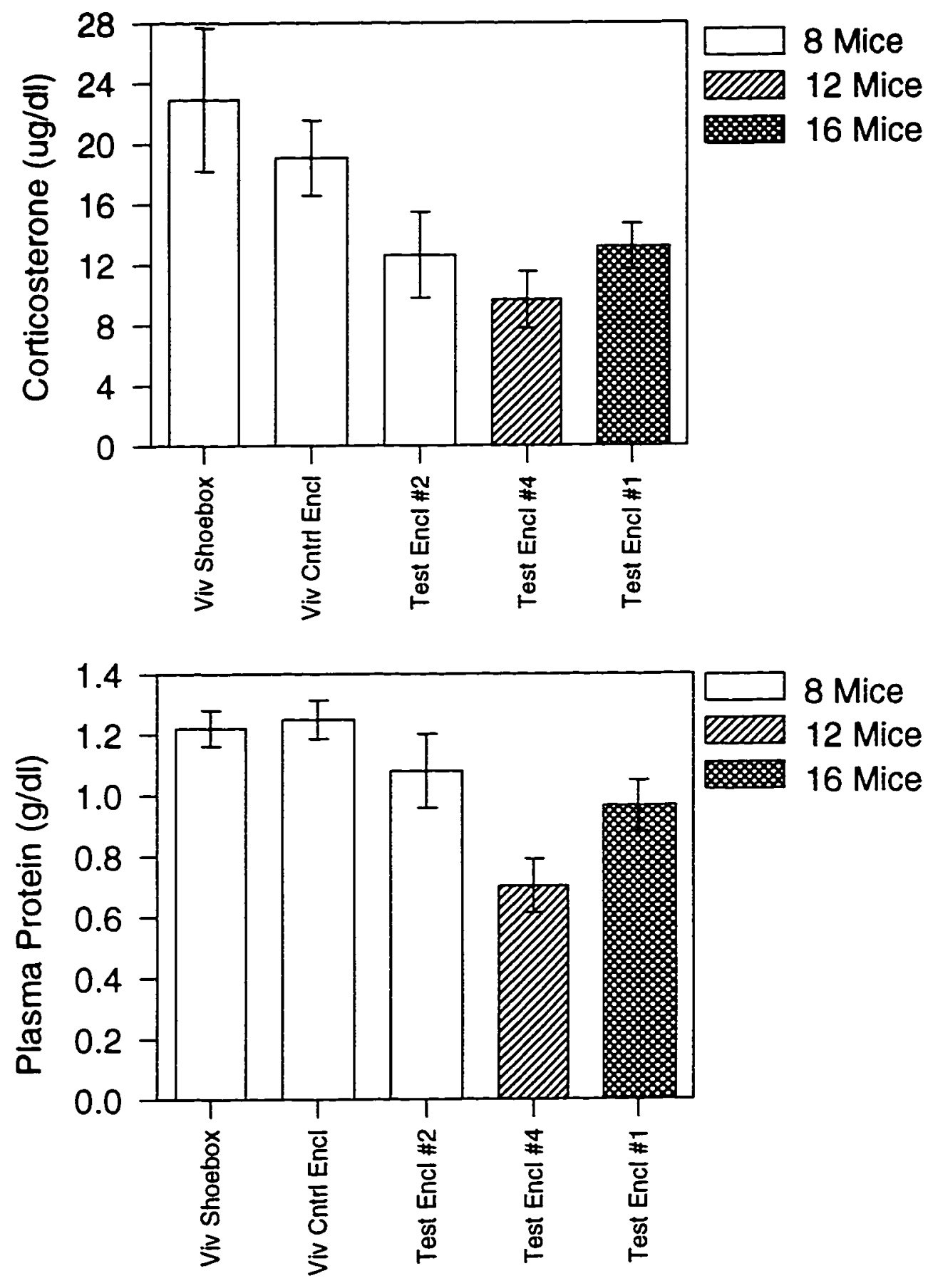
Figure 34 (Experiment 2): Mean \pm S.E.M. total leukocyte count and mean \pm S.E.M. eosinophil count. (See also Table 31).
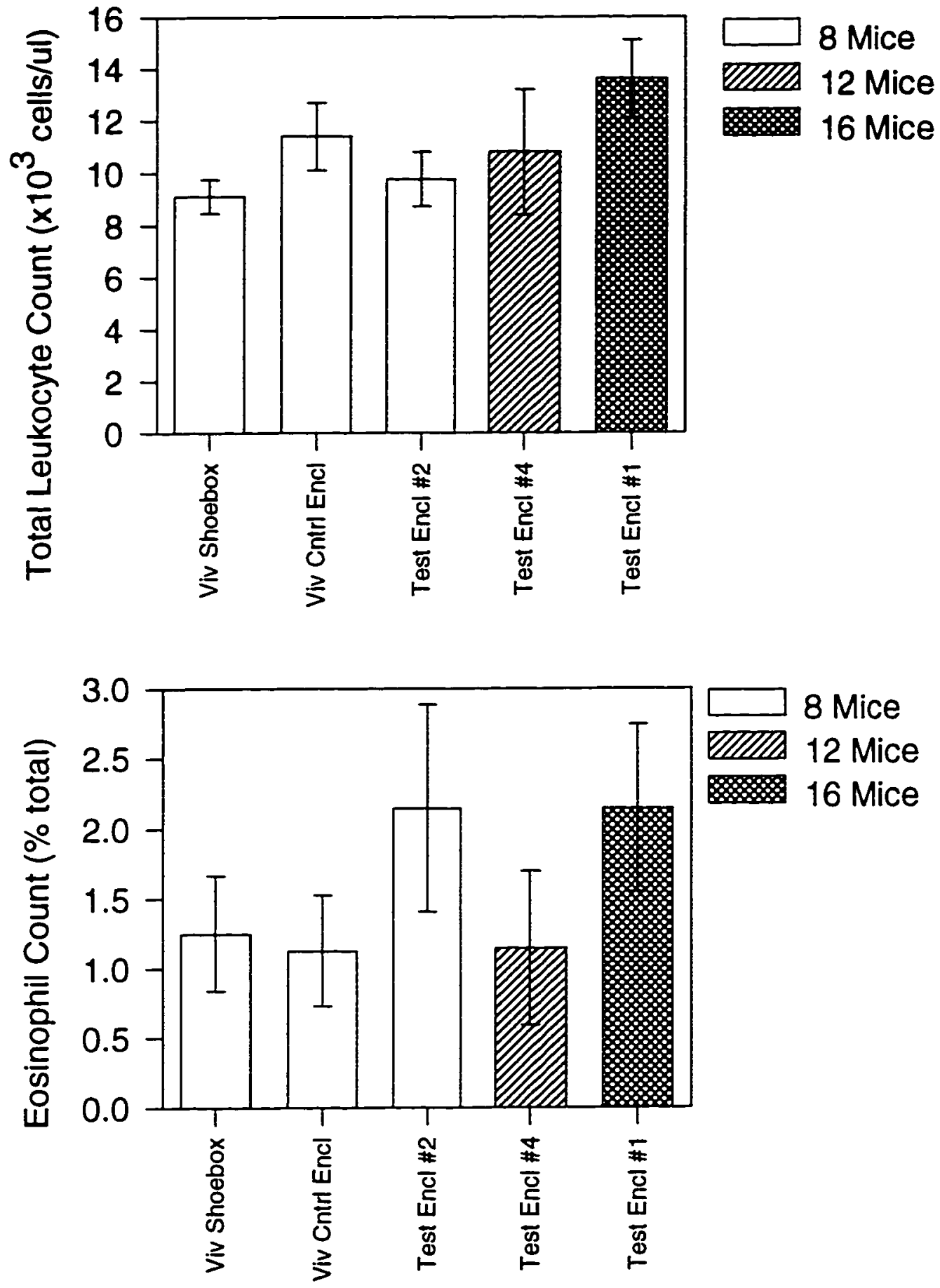
Figure 35 (Experiment 2): Mean \pm S.E.M. neutrophil count and mean \pm S.E.M. monocyte count(See also Table 31).
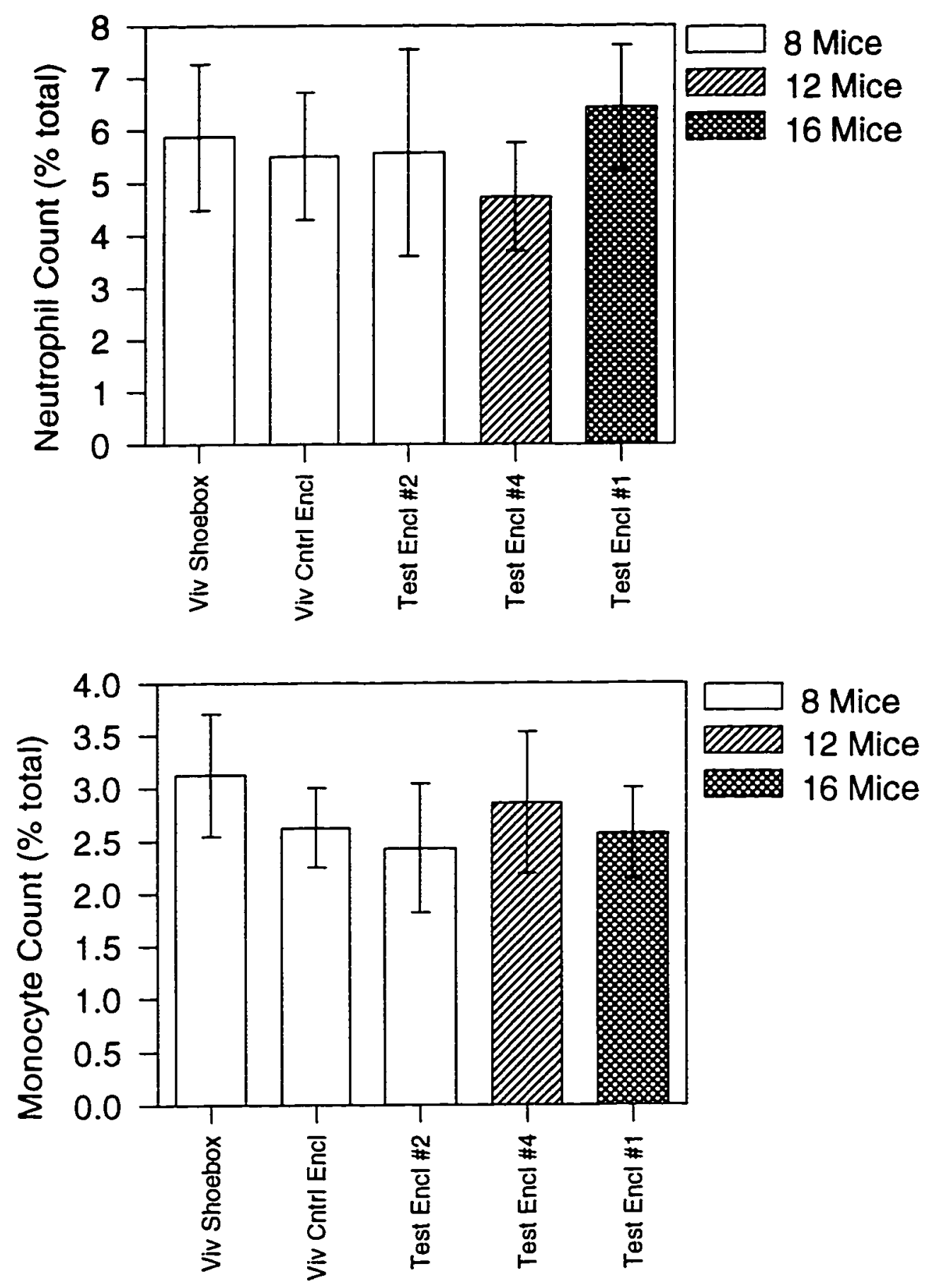
Figure 36 (Experiment 2): Mean \pm S.E.M. lymphocyte count and mean \pm S.E.M. neutrophil to lymphocyte ratio(See also Table 31).
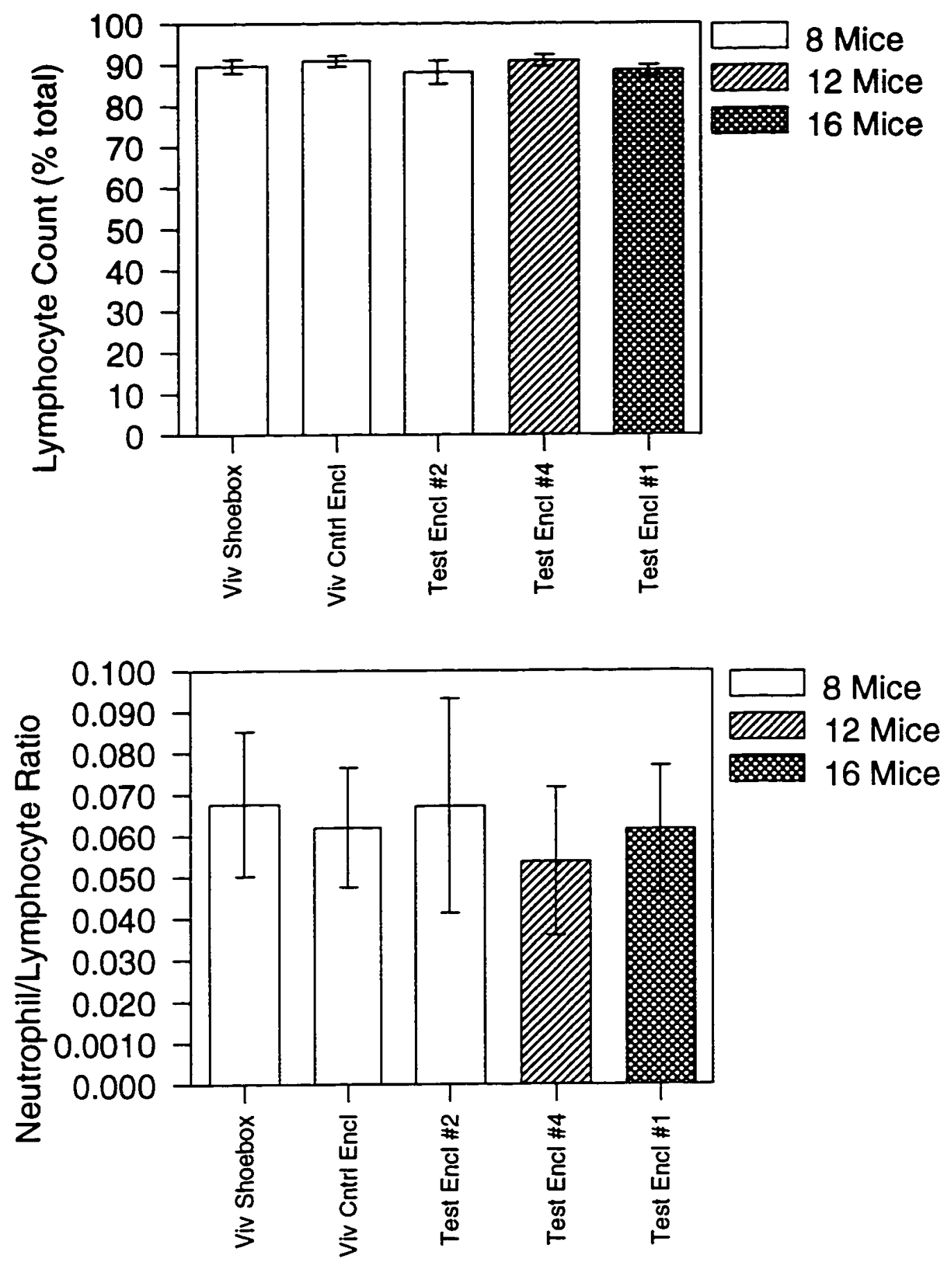
Table 32 (Experiment 2). Video scoring data used for behavioral evaluation. The observer scored twenty minutes of video per hour for two hours. Scores represent the total number of times any mouse engaged in the particular activity summed over all quadrants.

Total Count for Each Behavior Category

\begin{tabular}{lcccccc}
\hline Number of mice & $\mathbf{0}$ & $\mathbf{1}$ & $\mathbf{2}$ & $\mathbf{3}$ & $\mathbf{4}$ & $\mathbf{5}$ \\
\hline Test Enclosure \#2, 8 mice & 84 & 10 & 218 & $\mathbf{4}$ & 0 & 21 \\
Test Enclosure \#4, 12 mice & 154 & 10 & 578 & 5 & 0 & 22 \\
Test Enclosure \#1, 16 mice & 147 & 14 & 447 & 5 & 0 & 41
\end{tabular}

Count Per mouse for Each Behavior Category

\begin{tabular}{lcccccc}
\hline Number of mice & $\mathbf{0}$ & $\mathbf{1}$ & $\mathbf{2}$ & $\mathbf{3}$ & $\mathbf{4}$ & $\mathbf{5}$ \\
\hline Test Enclosure \#2, 8 mice & 10.5 & 1.2 & 27.2 & 0.5 & 0 & 2.6 \\
Test Enclosure \#4, 12 mice & 12.8 & 0.8 & 48.2 & 0.4 & 0 & 1.8 \\
Test Enclosure \#1, 16 mice & 9.2 & 0.9 & 27.9 & 0.3 & 0 & 2.6
\end{tabular}

Behavioral Score Category Definitions

0 - sleeping, still, sniffing

1 - eating, drinking

2 - grooming, moving

3 - social interaction (chasing or wrestling)

4 - aggressive behavior (biting)

5 - running 
Table 33 (Experiment 2). Adjusted G for ANOVA 1 from the test for normally distributed data and Burr-Foster q-test results for homogeneity of variances. A single asterisk indicated data that is not normally distributed $\left(G_{a d j}>\chi^{2} .05(\mathrm{~m})\right.$. Two asterisks indicates

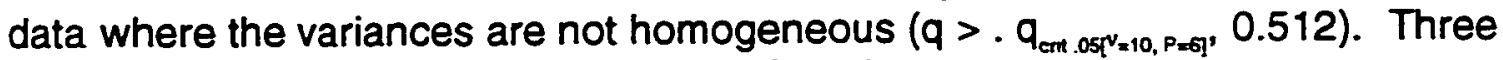
asterisks indicate a significant difference found.

\begin{tabular}{|c|c|c|c|c|c|c|}
\hline & $G_{\text {adi }}$ & $v$ & $x^{2}-0.05[v]$ & $q$ & Test Used & Results \\
\hline Initial Wt (grams) & 4.887 & 9 & 16.919 & 0.456 & ANOVA & \\
\hline Final Wt (grams) *. & 7.165 & 8 & 15.507 & 0.641 & & \\
\hline sqrt(Final Wt (grams)) ** & 7.656 & 9 & 15.507 & 0.697 & & \\
\hline Gain (grams) & 18.704 & 11 & 19.675 & 0.501 & ANOVA & \\
\hline $\arcsin (\operatorname{sqr}($ Gain (\%body))) & 9.333 & 9 & 16.919 & 0.410 & ANOVA & \\
\hline Heart Wt (grams) - & 22.440 & 10 & 18.307 & 0.409 & N/A & \\
\hline sqrt(Heart Wt (grams)) & 11.598 & 9 & 18.307 & 0.473 & ANOVA & $* *$ \\
\hline $\arcsin (\operatorname{sqr}($ Heart Wt (\%body))) & 3.601 & 9 & 16.919 & 0.354 & ANOVA & $\cdots$ \\
\hline Kidney Wt (grams) & 14.258 & 9 & 16.919 & 0.390 & ANOVA & \\
\hline $\arcsin ($ sqrt(Kidney Wt (\%body))) & 13.464 & 9 & 16.919 & 0.378 & ANOVA & \\
\hline Adrenal Wt (grams) & 10.363 & 8 & 15.507 & 0.417 & ANOVA & \\
\hline $\arcsin ($ sqrt(Adrenal Wt (\%body))) & 9.006 & 8 & 15.507 & 0.349 & ANOVA & \\
\hline Spleen Wt (grams) & 6.040 & 9 & 16.919 & 0.346 & ANOVA & \\
\hline $\arcsin ($ sqrt(Spleen Wt (\%body))) & 8.455 & 10 & 18.307 & 0.335 & ANOVA & \\
\hline Thymus Wt (grams) & 2.886 & 9 & 16.919 & 0.419 & ANOVA & \\
\hline $\arcsin ($ sqrt(Thymus Wt (\%body))) & 5.671 & 9 & 16.919 & 0.403 & ANOVA & \\
\hline Plasma Corticosterone & 13.055 & 8 & 15.507 & 0.353 & ANOVA & \\
\hline Plasma Protein & 10.034 & 9 & 16.919 & 0.336 & ANOVA & *** \\
\hline Total White Blood Count & 13.820 & 8 & 15.507 & 0.481 & ANOVA & \\
\hline $\arcsin ($ sqrt(Eosinophil (\% total))) & 25.063 & 9 & 16.919 & 0.437 & & \\
\hline $\arcsin ($ sqrt(Neutrophil (\% total))) & 6.911 & 9 & 16.919 & 0.384 & ANOVA & \\
\hline $\arcsin ($ sqrt(Monophil (\% total))) & 3.957 & 9 & 16.919 & 0.432 & ANOVA & \\
\hline $\arcsin (\operatorname{sqr}($ Lymphocyte $(\%$ total $)))$ & 12.295 & 9 & 16.919 & 0.420 & ANOVA & \\
\hline Neutrophillyymphocyte & 10.819 & 9 & 16.919 & 0.435 & ANOVA & \\
\hline
\end{tabular}


Table 34 (Experiment 2). Adjusted $G$ for ANOVA 1 from the test for normally distributed data and Burr-Foster q-test results for homogeneity of variances. A single asterisk indicated data that is not normally distributed $\left(G_{a d j}>\chi^{2} .05(y)\right.$. Two asterisks indicates

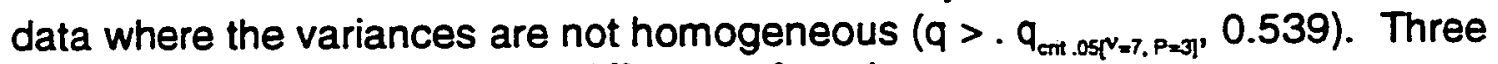
asterisks indicate a significant difference found.

\begin{tabular}{|c|c|c|c|c|c|c|}
\hline & $\underline{G}_{\text {adj }}$ & $v$ & $x^{2} \underline{0.05[v]}$ & $q$ & Test Used & Results \\
\hline Initial Wt (grams) & 9.819 & 9 & 16.919 & 0.465 & ANOVA & \\
\hline Final Wt (grams) & 12.578 & 10 & 18.307 & 0.514 & ANOVA & \\
\hline Gain (grams) & 14.894 & 10 & 18.307 & 0.518 & ANOVA & \\
\hline $\arcsin (\operatorname{sqr}($ Gain (\%body))) & 10.093 & 8 & 15.507 & 0.435 & ANOVA & \\
\hline Heart Wt (grams) & 6.669 & 9 & 16.919 & 0.381 & ANOVA & *** \\
\hline $\arcsin ($ sqrt(Heart Wt (\%body))) & 7.089 & 8 & 15.507 & 0.357 & ANOVA & $\cdots$ \\
\hline Kidney Wt (grams) & 3.175 & 8 & 15.507 & 0.390 & ANOVA & \\
\hline $\arcsin ($ sqrt(Kidney Wt (\%body))) & 5.572 & 10 & 18.307 & 0.486 & ANOVA & \\
\hline Adrenal Wt (grams) & 4.219 & 9 & 16.919 & 0.394 & ANOVA & \\
\hline $\arcsin (s q r($ Adrenal Wt (\%body))) & 2.957 & 9 & 16.919 & 0.402 & ANOVA & \\
\hline Spleen Wt (grams) & 14.187 & 9 & 16.919 & 0.360 & ANOVA & $\cdots$ \\
\hline $\arcsin ($ sqrt(Spleen Wt (\%body))) & 6.446 & 8 & 15.507 & 0.371 & ANOVA & $\cdots$ \\
\hline Thymus Wt (grams) & 3.691 & 10 & 18.307 & 0.402 & ANOVA & \\
\hline $\arcsin (\operatorname{sqt}($ Thymus Wt (\%body))) & 7.407 & 9 & 16.919 & 0.431 & ANOVA & \\
\hline Plasma Corticosterone & 6.153 & 9 & 16.919 & 0.450 & ANOVA & \\
\hline Plasma Protein & 13.481 & 8 & 15.507 & 0.493 & ANOVA & \\
\hline Total White Blood Count & 8.594 & 10 & 18.307 & 0.411 & ANOVA & \\
\hline $\arcsin (\operatorname{sqn}($ Eosinophil $(\%$ total $))){ }^{*}$ & 19.261 & 9 & 16.919 & 0.366 & Kruskall-Wallis & \\
\hline $\arcsin ($ sqrt(Neutrophil (\% total))) & 7.707 & 9 & 16.919 & 0.393 & ANOVA & \\
\hline $\arcsin ($ sqrt(Monocyte (\% total))) & 9.494 & 8 & 15.507 & 0.407 & ANOVA & \\
\hline $\arcsin ($ sqrt (Lymphocyte (\% total))) & 3.598 & 8 & 15.507 & 0.455 & ANOVA & \\
\hline Neutrophil/Lymphocyte & 8.930 & 9 & 16.919 & 0.419 & ANOVA & \\
\hline
\end{tabular}




\section{DISCUSSION OF SAB, EXPERIMENT 1 AND EXPERIMENT 2}

\section{Stress Assessment Battery}

Two weeks exposure to 3 hours/day restraint stress resulted in significant changes in body weight, thymus weight, spleen weight, and plasma protein concentration.

There was significantly less growth (weight gain) in the stress group than in the control group, even though there was very little difference in the food and water consumption between the two groups. This supports the assumption that the difference in weight gain can be attributed to the restraint treatment imposed on the stress group and the altered physiology resulting from this treatment.

The lower thymus weights and spleen weights in the stress group may be indicative of immunosuppression. This would be consistent with chronic hypercorticosterone secretion. While the differences in plasma corticosterone concentrations were variable and not statistically significant, the mean plasma corticosterone concentration was higher in the stress group. This trend is also consistent with chronic hypercorticosterone secretion.

The higher plasma protein concentration in the stress group is difficult to interpret without a more complete analysis of the differential blood protein profile. There were no significant changes in total white blood cell counts, total eosinophil counts and the neutrophil to lymphocyte ratio. In addition, the pathology report indicated that there was no evidence of any stomach ulcerations in any of the mice. These results, along with the fact that there was little difference in mean adrenal weights between the stress and control groups, leads us to believe that this stress response was not a strong one. Perhaps the type of stressor used was not severe enough or the period of stress exposure was not long enough. Also, the number of animals in each experimental group may not have been great enough to resolve small changes given the variability that was noted. Finally, it is also possible that some adaptation to the 3 hour restraint occurred over the 14 day exposure period. 


\section{Experiment 1}

There were no trends seen with increasing population density in most of the parameters of the SAB in Experiment 1. Statistical analysis indicated that there were significant differences seen in plasma corticosterone and kidney weights. The mean plasma corticosterone levels were highest in the cages housing 16 mice and lowest in the cages housing 8 mice. Normalized kidney weights were lowest in the cages housing eight mice.

The results from the SAB performed on the animals in Experiment 1 were, unfortunately, inconclusive. Food bars in the test enclosures came loose in some of the cages. This could have happened because the bars were old and beginning to dry out or because they were not prepared and glued to the plates properly. In any case, these fallen food bars impeded the filtering system and became contaminated with urine thus providing a constant source of odor contamination. The ammonia levels in the affected cages were consistently high and, therefore, became a confounding variable in this study. High ammonia levels have been shown to be a stressor that can play a significant role in compromising the health of rodents (Broderson, Lindsey and Crawford, 1976 and Schaerdel et al., 1983) with the most frequently reported toxic effect of ammonia involving the respiratory tract.

Ammonia is produced by the action of urea-splitting bacteria on urine. Specifically, bacterial ureases catalyze the hydrolysis of urea to ammonia and carbamate (Collins and D'Orazio, 1993) while a second series of enzymes, D- and Lamino acid oxidases can deaminate amino acids to their corresponding keto acid and ammonia. Adverse effects of ammonia include aggravation of respiratory infections (Broderson, Lindsey and Crawford, 1976) subclinical histologic changes in tracheal epithelium (Gamble and Clough, 1976), decreased immune function (Targowski, Klucinski and Babiker, 1984) and impairment of hepatic drug metabolism (Veseli, Lang and White, 1973). In addition, it has been shown to affect some of the parameters of the $S A B$ such as adrenal weights, plasma corticosterone concentration, peripheral 
lymphocyte populations and overall growth in mice (Peters and Festing, 1990 and Peng, et al., 1989) .

Population density has been shown to be positively correlated with intracage ammonia levels (Serrano, 1971). While there is no definitive rule as to the duration and concentration of ammonia that will induce pathological changes mice, Broderson et al. (1976) showed that rats exposed to $200 \mathrm{ppm}$ of ammonia for four and eight days developed sub-clinical pathological changes ranging from mild thickening through gross stratification of the tracheal epithelium, to cilia loss and extensive mucuos membrane folding.

The effect of environmental ammonia challenging the well-being of the mice can be seen in this study if we compare the animals housed in the test enclosures without the filtering system. The mean plasma corticosterone level of the mice in this cage was greater than that of the mice in the vivarium shoe-box cage control and and the testenclosure with the filtering system, all housing similar population densities of 8 . Also, these mice had the lowest mean total leukocye counts and the greatest heart weights when compared to the other two cages housing 8 mice.

As such, the few significant differences seen in the cages housing 16 mice can not be interpreted as being due to the effect of the increased population density. The experiment was, therefore, repeated (Experiment 2) with precautions made so that the confounding variable introduced by the fallen food bars was minimized. 


\section{Experiment 2}

In this experiment the number of mice per Test Enclosure affected the amount of water used per mouse per day, heart weight, and plasma protein concentration. Mice in the Test Enclosure housing 16 mice used less water than mice in the Test Enclosure housing 8 mice. Heart weights of mice in the Test Enclosures housing 12 and 16 mice were greater than those of mice in the Test Enclosure housing 8 mice. The mean normalized heart weights of the mice Test Enclosure housing 16 mice was greater than those of mice in the Test Enclosures housing 8 animals. Mice in the Test Enclosure housing 12 mice had greater heart weights and lower plasma protein concentrations than mice in the Test Enclosure housing 8 mice.

Interpretation of the effects of the number of mice per cage in this study has its complications. Reduced water consumption by the 16 mouse Test Enclosure mice corresponded to and could be due to the reduced amount of food consumed (Dr. Sig Rich, campus vetinarian, personal communication). The 16 mouse Test Enclosure mice probably ate less because of the improved thermal efficiency of a 16 mouse huddle. Greater heart weights of 16 and 12 mouse Test Enclosure mice (15\% and $12 \%$ ) can be partially explained by the greater body weights of those mice ( $4 \%$ and $5 \%$ ). The experimental results are further complicated by the presence of ammonia and methylamine only in the 12 mouse Test Enclosure (which were not caused by fallen bars in this study).

In any case, the differences in plasma corticosterone and kidney weights seen in the cage housing 16 mice in Experiment 1 is not seen in Experiment 2. This suggests odor contamination from fallen food bars in the previous study was not a confounding variable in this study.

In conclusion, these findings indicate that mice housed in AEM simulators at densities of 8,12 or 16 per simulator are similar in terms of their growth, physiology and behavior. After 30 days, there was no evidence of compromised health or well-being in any of the 3 housing densities. 


\section{REFERENCES}

Breazile, J.E., "Physiological basis and consequences of distress in animals", JAVMA, 1987, 191(10):1212-1215.

Broderson, J.R., Lindsey, J.R. and Crawford, J.E., "The role of environmental ammonia in respiratory mycoplasmosis of rats" American Journal of Pathology, 1976, 85(1):115127.

Canon,W.B., " Organization for physiological homeostasis", Physiology Review, 1929, 9:399-431.

Christian, J.J, "The pathology of overpopulation", Military Medicine, 1963, 128:571-603.

Christian, J.J, "Physiological and Pathological Correlates of Population Density", Proceedings of the Royal Society of Medicine, 1964, 57:169-174.

Christian, J.J and Davis, D.E. "Endocrines, Behavior and Population", Science, 1964, 146:1550-1560.

Collins, C.M. and D'Orazio, S.E.F., "Bacterial ureases: structure, regulation of expression and role in pathogenesis", Molecular Microbiology, 1993,9:907-913.

Daniels-Severs, A., Goodwin, A., Keil, L.C. and Vernikos-Danellis, J., "Effects of chronic crowding and cold stress and their relations to pharmacological actions", Pharmacology, 1973, 9:348-356.

deCatanzaro, D. and Graham, C., "Influences of exogenous epinephrine on two reproductive parameters in female mice: disruption of receptivity but not implantation", Hormones and Behavior, 1992, 26(3):330-338.

Edwards, E.A. and Dean, L.M., "Effects of crowding of mice on humoral antibody formation and protection to lethal antigenic challenge". Psychosomatic Medicine, 1977, 39(1):19-24.

Gamble, M.R. and Clough, G., "Ammonia build-up in animal boxes and its effect on rat tracheal epithelium", Lab Animals, 1976, 10:93-104. 
Garvy, B.A., King, L.E., Telford, W.G., Morford, L.A. and Fraker, P.J., "Chronic elevation of plasma corticosterone causes reductions in the number of cycling cells of the B lineage in murine bone marrow and induces apoptosis", Immunology, 1993, 80(4):587-592.

Griffiths, N.S. and Anisman, H., "Norepinephrine and serotonin alterations following chronic stressor exposure: mouse strain differences", Pharmacology. Biochemistry and Behavior, 1994, 49(1):57-65.

Guide for Care and Use of Laboratory Animals, Public Health Service, National Institutes of Health, NIH Publication, No. 85-23, 1985.

Hardy, C., Quay, J., Livnat, S. and Ader, R., "Altered T-lymphocyte response following aggressive encounters in mice" Physiology and Behavior, 1990, 47:1245-1251.

Hermann, G., Beck, F.M. and Sheridan, J.F., "Stress-induced glucocorticoid response modulates mononuclear cell trafficking during an experimental influenza viral infection", Journal of Neuroimmunology, 1995, 56:179-186.

Jain, N.C., Schalm's Veterinary Hematology 4th edition, Lea and Febiger, 1986.

Lloyd, J.A. and Christian,J.J., "Reproductive activity of individual females in three experimental freely growing populations of house mice (Mus musculus)", Jeurnal of Mammalogy, 1969, 50(1):49-59.

Miller, D.B. and Chernoff, N., "Restrain-induced stress in pregnant mice-degree of immobilization affects indices of stress and developmental outcome in offspring", Toxicology, 1995, 98(1-3):177-186.

Moberg, G.P., Animal Stress, 1985, American Physiological Society, pp 27-49.

Moberg, G.P., "Problems in defining stress and distress in animals", JAVMA, 1987, 191(10):1207-1211.

Munck, A., Guyre, P.M. and Holbrook, N.J., "Physiological functions of glucocorticoids in stress and their relations to pharmacological actions", Endocrine Reviews, 1984, $5(1): 25-44$.

Peng, X., Lang, C.M. and Drozdowicz, C.K., "Effect of cage population density on plasma corticosterone and peripheral lymphocyte populations of laboratory mice", Lab Animals, 1989, 23:302-306. 
Peters, A. and Festing, M., "Population density and growth rate in laboratory mice", Lab Animal Science, 1990, 24:273-279.

Schaerdel, A.D., White, W.J., Lang, C.M., Dvorchik, B.H. and Bohner, K., Localized and systemic effects of ammonia in rats", Laboratory Animal Sciences. 1983, 33(1) 40-45.

Selye, H., "A syndrome produced by diverse nocuous agents" Nature London, 1936, 138:32.

Serrano, I.J., "Carbon Dioxide and ammonia in mouse cages: effect of cage covers, population and activity", Lab Animal Science, 1971, 21:75-85.

Svec, F., Goron,S. and Tate, D., "Glucocorticoid receptor regulation: the effects of adrenalectomy, exogenous glucocorticoid, and stress on hepatic receptor number in male and female mice", Biochemical Medicine and Metabolic Biology, 1989, 41:224233.

Targowski,S.P., Klucinski, W. and Babiker, S., "Effect of ammonia on in vivo and in vitro immune responses", Infectious Immunity, 1984,43(1);289-293.

Tepperman, J., and Tepperman, H.M., Metabolic and Endocrine Physiology, 5th edition, Year Book Medical Publishers, Inc, 1987.

Veseli, E.S., Lang, C.M. and White, W.J., "Hepatic drug metabolism in rats: impairment in a dirty environment" Science, 1973, 179: 896-897.

Welch, B.L. and Welch, A.S., "Sustained effects of brief daily stress (fighting) upon brain and adrenal catecholamines and adrenal, spleen and heart weights of mice", Proceedings of the National Academy of Science, 1969, 64:100-107.

Zha, H., Ding, G. and Fan, S., "Serum factor(s) induced by restraint stress in mice and rats suppresses lymphocyte proliferation "Brain. Behavior and Immunology, 1992, 6(1): 18-31. 